\title{
NUCLEAR MATERIALS RESEARCH PROGRESS REPORTS
}

FOR 1978

\author{
Materials and Molecular Research Division \\ Lawrence Berkeley Laboratory \\ university of California \\ Berkeley, California 94720
}

D. R. Olander,

Principal Investigator 
CONTENTS

1. Iodine Stress Corrosion Cracking of zircaloy,

by S. Shann... . . . . . . . . . . . . . . . 1 1-34

2. Reduction of $\mathrm{UO}_{2}$ and Measurement of the

Oxygen-Metal Ratio, by K. Kim. . . . . . . . . . . . . 35-43

3. The Surface Chemistry of Epitaxial Silicon

Deposition by Thermal Cracking of Silane,

hy M. Farnaam . . . . . . . . . . . . . . . . . . 44-49

4. Kinetics of Laser Pulse Vaporization of $\mathrm{vO}_{2}$,

by C. H. Tsai. . . . . . . . . . . . . . . . . 50-60

5. Retention and Release of Water Vapor by Uranium Dioxide

by D. Sherman . . . . . . . . . . . . . . . 61-79

6. Thermal Gradient Migration of Metallic Inclusions in $\mathrm{UO}_{2}$ '

by R. Yang . . . . . . . . . . . . . . . . 80-99

7. Molecular Beam Studies of Atomic Hydrogen

Reduction of Oxides, by D. Dooley . . . . . . . . . 100-105 
IODINE STRESS CORROSION CRACKING OF ZIRCALOY

by Shih-Hsiung Shann

[Decombor 1978]

\section{INTRODUCTION}

The outstanding high temperature water corrosion resistance and highly desirable nuclear characteristics of zircaloy have made it an excellent cladoing material of light water reactors. However, low-ductility failures of the cladding still occasionally occur, pointing to the need for understanding of the fracture mechansim and prevention methods.

The temperature range of fuel cladding eliminates the possibility of liquid metal embrittlement( 7$)$, and the fractography of cracked cladding resembles that of stress corrosion cracking in the laboratory (7). Previous works (1-12, 23, 24) have been studied mainly unirradiated specimens in iodine and wood et.al. (6.11) tested irradiated samples, but nothing concerning simultaneous irradiation and stres:i corrosion has been reported.

Iodine is present as low yield fission product in irradiated fuel rods. However, according to thermodynamics, all of this iodine should be combined with the more abundant fission product cesium to produce the stable solid CsI. This substance is not an active stress corrosion cracking agent to zircaloy, as demonstrated by out-of-pile tests $(8,23)$. The main purpose of this project is to determine how iodine is released from CsI inside the fuel rod in order to activate the stress corrosion cracking process. Our hypothesis is that the strong radiation field inside the fuel rod decomposes the thermodynamically stable CsI (14) and liberates enough free iodine to permit stress corrosion cracking to occur. To achieve this long-range objective, establishment of a complete icdine-zircaloy stress corrosion cracking data base is necessary for a reference system and for better understanding of fracture mechanism.

II. EXPERIMENTAL

The present experiment utilizes a tube-burst (biaxial) test mode. One half 
inch OD tube samples (ground to $10 \mathrm{mil}$ wall thickness) internally pressurized by argon are connected to a pressure transducer (Statham PA-891-3M) and a filling valve (Fig. 1). The assembly is placed in an enclosure and heated resistively.

For temperature measurement and control, a thermocouple is pushed against the zircaloy tube by a stainless steel spring, the force of which is enough for the good contact between thermocouple and zircaloy, but is too small to cause indentation of the tube. Thin thermocouple wire $(0.01$ " diameter) is used in order to keep the heat loss through thermocouple wires as small as possible. An electronic control system (Omega Engineer, Inc. Model 49) has been installed to compare the output voltage of the thermocouple with nzeset standard and to operate a relay in order to turn on or turn off the heating current. The temperature can be controlled within $\pm 5^{\circ} \mathrm{C}$ of the set temperature. The tube is pressurized by an inert gas prior to closing the filling valve and inserting into the vacuum system. The initial hoop stress in the zircaloy tube is calculated from the thin-wall tube formula

$$
\sigma_{0}=\frac{P R}{w}
$$

where $\sigma_{0}=$ initial hoop stress

$$
\begin{aligned}
& P=\text { internal gas pressure } \\
& R=\text { tube radius } \\
& W=\text { tube wall thickness }
\end{aligned}
$$

As the crack propagates, the net section thickness (initial wall thicknesscrack length) decreases, and the stress increases. To prevent damage to the diffusion pump following a burst of the internally pressurized test specimen, a loose-fitting glass rod is inserted in the tube specimen to reduce the gas volume. When the specimen fails, <0.0l moles of gas fiow into the vacuum system. 
Molecular iodine is supplied to the surface through $4.57 \mathrm{~mm}$ diameter tubing (doser) which is $2-5 \mathrm{~mm}$ from the specimen surface. With this delivery system, iodine impinges on a spot $\sim 5 \mathrm{~mm}$ in diameter on the outside surface of the zircaloy specimen.

The iodina flow rate $\ell$ * (moles/sec) through the doser is calculated from Shock's model (17) for flow through small diameter, long tubes.

$$
\frac{L}{D}=\frac{\sqrt{\frac{1}{2} \pi}}{64}\left[\left(\dot{m}^{-1}-\dot{m}+2 \dot{m} \log \dot{m}\right) p_{1}+22(1-\dot{m})^{2} / \dot{m}\right]
$$

where:

$$
\begin{aligned}
D & =\text { diameter of doser tube } \\
L & =\text { length of doser tube } \\
\dot{m} & =\text { mass flow rate } \\
P_{1} & =\text { dimensionless pressure at tube inlet } \\
P_{1} & =\frac{P_{1}}{\sqrt{\frac{1}{2} \pi R T}(\mu / D)} \\
P_{1} & =\text { pressure at tube inlet (iodine reservoir) } \\
\mu & =\text { viscosity }
\end{aligned}
$$

The intensity $I^{*}$ (moles/ $\mathrm{cm}^{2} \mathrm{sec)}$ ct iodine impinging on the surface of the tube at a distance $d$ from the outlet of doser is given by

$$
I *=\frac{X(\dot{m} / 254)}{\pi d^{2}}
$$

where $X$ is the peaking factor (18), which characterizes noncosine emission from long channels.

The equivalent pressure of iodise at the zircaloy suriace $\left(\mathrm{P}_{\mathrm{Eq}}\right)$ is calculated from

$$
I *=\frac{P_{\mathrm{eg}}}{\sqrt{2 \pi \mathrm{mk} T}}
$$


where $\quad m=$ molecular weight of iodine

$k=$ gas constant

$\mathbf{T}=$ temperature

Two lots of stress relieved zircaloy-2 were tested. One lot was obtained from Stanford Research Institute with unknown lot number $10.0 .=0.503^{\prime \prime}, \mathrm{I} . \mathrm{D}$. = $0.453^{\prime \prime)}$, the other lot was purchased from Sandvik Special Metals, lot number $9 \mathrm{AX} 32$ (O.D. $\left.=0.486 \pm 0.002^{\prime \prime}, \mathrm{I} . \mathrm{D} .=0.414^{\prime \prime} \pm 0.002^{\prime \prime}\right)$. Table 1 gives the chemical composition.

III. RESULTS

\section{A. Failure Modes}

With iodine, specimens exhibited pinhole-type failure (Figs. 2-6), which was completely different from the burst-type failure which occurs in the absence of iodine (Fig. 7).

The Sandvik specimens, with stres!s level between $54 \mathrm{ksi}$ and $42 \mathrm{ksi}$, all Eractured in the pinhole mode. Three specimens with stress $>55 k s i$ fracturea in non-pinhole mode, even in the presence of iodine.

The Eractography of pinhole and rluctile failures are completely differunt. Fig. 8 is a scanning electron micrograph bi a pinhole type failure. Fig. 9 is for ductile failure. Fig. 10 shows that besides the penetrating cracks, there are a number of non-penetrating cracks on the outside surface in the iodine-affected region. There are numerous small cracks branching out from main crack at an angle to main crack propagation direction (Fig. 11). As the main crack propagates, the remaining intact wall thickness decreases, so the net section stress increases. After the crack length reaches a critical length, a transition to ductile failure occurs (Fig. 12) when the net section stress reaches the ultimate tensile stress and the tube fails immediately. A few grains fail by cleavage, as shown in Fig. 13. Fig. 14 is a detail of Fig. 13. 


\section{B. Time to Rupture}

The results from unflawed specimens from SRI are plotted in Fig. 15. Preflawed (0.001" depth, 1 " long notches) data are shown in Fig. 16. Stress corrosion cracking was observed at iodine pressure as low as $10^{-3}$ torr.

Control specimen results for the Sandvik tubes at $300^{\circ} \mathrm{C}$ are shown in Fig. 17. Fig. 18 gives the time-to-failure versus temperature for 0.05 tor $r$ iodine pressure. Fig. 19 and 20 record the times to failure versus stress for two different iodine pressures. Fig. 21 is a time-to-failure/equivalent iodine pressure relationship.

The indine pressure necessary to cause stress corrosion cracking is much less than previously reported(19).

IV. DISCUSSION

\section{A. Iodine concentration}

Most previous works use $\mathrm{mg} / \mathrm{cm}^{2}$ as a unit to describe the iodine potential. According to basic chemistry, the partial pressure is the correct masure of chemical potential. Une(19) performed experiments with different volume-to-surface ratios, keeping one variable constant and changing the other. The influence of two variables on the time to failure was recorded. He concluded thit in zircaloy stress corrosion cracking, iodine partial pressure (e.g., torr), not the surface density $\left(\mathrm{mg} / \mathrm{cm}^{2}\right)$ is the correct unit for correlating the stress corrosion cracking phenoemnon.

Let us consider the experimental methods for establishing the iodine concentration used in previous investigations:

(a) A fixed amount of iodine is sealed in the inside of zircaloy tube (23). In this case, there is considerable surface other than zircaloy on which iodine can plate out. In addition, only the zircaloy is heated and the iodine will condense on the cold regions of the system. The actual iodine 
responsible for stress corrosion cracking is much lower than what is reported.

(b) A fixed amount of iodine is sealed inside the zircaloy tube, and the tube is plugged at both ends $(1,4,5,8,24)$, or iodine is sealed with zircaloy in closed system, as in some mandrel tests $(6,7,11)$. In those cases, the amount of iodine first decreases with time, After certain period, there may be an equilibrium between iodine and zircaloy. But we do not know what the equilibrium iodine pressure is, or how long it will take to achieve the equilibrium.

From the discussion above, it is clear that in previous investigations, insufficient attention has been given to meintaining contant, known iodine concentration or even using the correct units to describe the iodine concentration.

\section{B. Biaxiality}

In our specimen assembly (Fig. 1), one end is free. Under internal pressure, the hoop-axial stress ratio is two (in other works, the ratio is between 0.5 and 1 (8) or not given). The yielding condition of anisotropic materials such as zircaloy under biaxial loading depends on the ratio of two principal stresses $(15,20)$, so a hoop stress measurement alone is not a sufficient condition for fixing the time to failure. The time to failure can change with different stress biaxiality ratios even though the hoop stress, iodine pressure, and temperature are fixed.

\section{Stress and Iodine Pressure Thershold}

One Sandvik specimen, held at $300^{\circ} \mathrm{C}$ and $47.6 \mathrm{kpsi}$, did not fail after 89 hours at an iodine pressure of .013 torr. A possible explation is that the stress was below the threshold stress for stress corrosion cracking for the particular iodine partial pressure used. Alternatively, the icdine pressure was 
below the threshold value for that stress level. The threshold concepts are accepted by most previous workers $(1-12,23)$. The threshold concept implies a change in fracture mechanism in narrow ranges of stress and iodine pressure, so that time to failure is greatly affected.

D. High Stress Failure

Three Sandvik specimens stressed near 55Ksi did not exhibit pinhole type failure under iodine. The argument that stress corrosion cracking can occur only within a limited stress range (21) can explain this phenomenon. According to this explanation, stress of $55 \mathrm{Ksi}$ is too high for pinhole-type stress corrosion cracking.

E. Data Correlation

Stress corrosion cracking of zircaloy is a low ductility fracture process (strain in the range of a few percent). If crack propagation dominates the lifetime of specimens, the model of slow crack growth under chemical attack for brittle solid may be applied to this situation(22).

In this model, the crack growth rate is described in chemical reaction rate terminology by:

$$
\frac{d a}{d t}=A\left[P_{e q}\right]^{n} \exp \left(-\frac{E^{\star}}{R T}\right) F(K)
$$

where

$$
\begin{aligned}
\mathbf{a}= & \text { crack length } \\
\mathbf{E}^{*}= & \text { activation energY of the chemical step responsible for stress } \\
& \text { corrosion } \\
\mathbf{n}= & \text { reaction order } \\
\mathbf{A}= & \text { constant }
\end{aligned}
$$

$F$ in Eq. (2) is the functional dependence of the crack growth rate on the stress intensity factor, which is 


$$
\begin{aligned}
& K=Y \sigma \sqrt{a} \\
& Y=\text { geometric factor }
\end{aligned}
$$

The hoop stress in the intact section of the wall is:

$$
\sigma=\sigma_{0} \frac{w}{w-a}
$$

where

$$
\begin{aligned}
& \sigma_{0}=\text { initial hoop stress (see eq. 1) } \\
& w=\text { initial wall thickness } \\
& w-a=\text { intact wall thickness }
\end{aligned}
$$

Integrating $\mathrm{Eq} 2$ :

$$
t_{f}=\frac{1}{A\left[P_{e q}\right]^{n}} \exp \left(\frac{E^{\star}}{R T}\right) \int_{a_{0}}^{a_{C}} \frac{d a}{F(K)}
$$

$a_{0}=$ initial length of the crack pre-existing through manufacture or machining $\left(a_{0} \simeq 0.22 \times 10^{-3} \mathrm{in}\right)$

$a_{c}=$ crack length at which the section stress is equal to the ultimate tensile stress:

$$
\frac{\sigma_{0}^{w}}{\left(w-a_{c}\right)}=\sigma_{\text {UTS }}
$$

Here it is supposed that stress corrosion propagates the crack from length $a_{0}$ to $a_{c}$ where ductile failure takes place very rapidly. This final process can be seen on the SEM picture of Fig. 12.

Least square curve fittion of the $T$ and $P_{\text {eq }}$ data gives:

$$
E^{\star}=7.12 \pm 0.9 \mathrm{Kcal} / \mathrm{mole}
$$




$$
\mathrm{n}=0.51 \pm .03
$$

Three crack velocity and stress intensity factor relations (22) were tried:

$$
\begin{aligned}
& F(K)=\exp \left(\frac{B K}{T},\right. \\
& F(K)=K^{m} \\
& F(K)=\exp (-B / K)
\end{aligned}
$$

where $B$ and $m$ are constants. These relationships all fjt the stress-dependence data to the same accuracy. $F(K)=k^{m}$ case gives $m=4.0 \pm 0.1$, which is consistent with the results of Tuck, et.al. (5) and Polan et.al. (24).

\section{PLANNED EXPERIMENTS}

A. Perform the experiment using $\mathrm{FeI}_{2}, \mathrm{AlI}_{3}, \mathrm{ZrI}_{4}$, and CsI, to see if these species, cause stress corrosion cracking.

B. Determine whether crack initiation or crack propagation is the slow ster. We: plan to use a camera to record the surface condition during the experiment, in order to determine when the surface crack first appears on the specimen. If this method does not work (i.e., if the resolution of the film is not good enough), we will have to use several specimens exposed to iodine for different time periods and removed them from the system for inspection of crack length.

c. Apply itress, CsI (or other corrosive agent), and radiation simultaneously to the zircaloy specimen to see if radiation liberates iodine and causes stress corrosion cracking. 


\section{REFERENCES}

1. C. C. Busby, R. P. Tucker and J. E. McCauley, J. Nucl. Mater., 55 (1975) 54-82.

2. A. Garlick and P. D. Wolfenden, J. Nucl. Mater., 4l (1971) 274-272.

3. P. H. Kreyns, G. L. Spahr and J. E. MCCanley, WAPD-TM-1203. (2976)

4. J. G. We inberg, WAPD-TM-1048. (1974)

5. R. P. Tucker, P. H. Kreyns and J. J. Kearns, WAPD-TM-1248:

6. J. C. Wood, J. Nucl. Mater., 45 (1972/1973) 105-122.

7. J. C. Wood, B. A. Surette, I. M. London and J. Baird, J. Nucl. Mater., 57, (1975) 155-197.

8. K. Videm and L. Lunde, Annals of Nucleur Energy, 3 (1976) 305-313.

9. I. Aitchison and B. Cox, Corrosion, 28 (1972) 83-87.

1n. B. Cox, Corrosion, 28 (1972) 207-217.

11. J. C. Wood, Nucl. Technology, 23 (1974) 63-79.

12. A. Garlick, J. Nucl. Mater., 49 (1973/74) 207-224.

13. K. Elayaperumal, P. K. De and J. Balachandra, J. Nucl. Mater., $45(1972,73)$ 323-330.

14. D. Cubicciotti and J. H. Davis, Nuclear Science and Engineering, 60 (1976) 314-31.9.

15. W. A. Backofon, W. F. Hosford, Jr. and J. J. Burke, Trans. ASM, 1962, $264-267$.

16. R. A. Gough, R. Lam, C. Martinez and D. Morris, Nuclear Instrumentis and Methods, 138 (1976) 415-419.

17. A. Schock, FSEC-NSG-217-74/37B (1974).

18. D. R. Olander and R. H. Jones, Entropie, No. 30, Nov./Dec. 1969, $42-47$.

19. Katsumi Une, J. of Nucl. Sci, and Tech., 14 (1977) 443-451.

20. E.I.H. Lin, 'Prans. ANS, 27 (1977) 597-599.

21. W. J. Baily, C. L. Wilson, L. J. MacGowan and P. J. Pankaskie, C00-4066-2, PNL-2488, UC-78 (1977).

22. A. G. Evans and T. G. Longdon, Progress in Materials Science, $213 / 4$ (1976). 
23. D. Cubicciotti and R. I. Jones, EPRI NP-717 (1978).

24. N. W. Polan aild R. P. Tucker, WAPD-1313 (1977). 
Table 1. Composition of Sandvik $\mathrm{Zr}-\mathrm{Z}$ (9AX-32)

\section{INGOT ANALYSIS}

\section{COMPOSITION IN PERCENT}

\begin{tabular}{|c|c|c|c|c|}
\hline & Spec. & Top & Middle & Bottom \\
\hline Sn & $1.20-1.70$ & 1.55 & 1.47 & 1.46 \\
\hline $\mathrm{Fe}$ & $0.07-0.20$ & 0.14 & 0.13 & 0.14 \\
\hline $\mathrm{Cr}$ & $0.05-0.15$ & 0.10 & 0.09 & 0.09 \\
\hline $\mathrm{Ni}$ & $0.03-0.08$ & 0.05 & 0.04 & 0.04 \\
\hline $\mathrm{Fe}+\mathrm{Cr}+\mathrm{Ni}$ & $0.18-0.38$ & 0.29 & 0.27 & 0.28 \\
\hline $2 \mathrm{r}$ & \multicolumn{3}{|c|}{ B A L A N C E } & \\
\hline
\end{tabular}




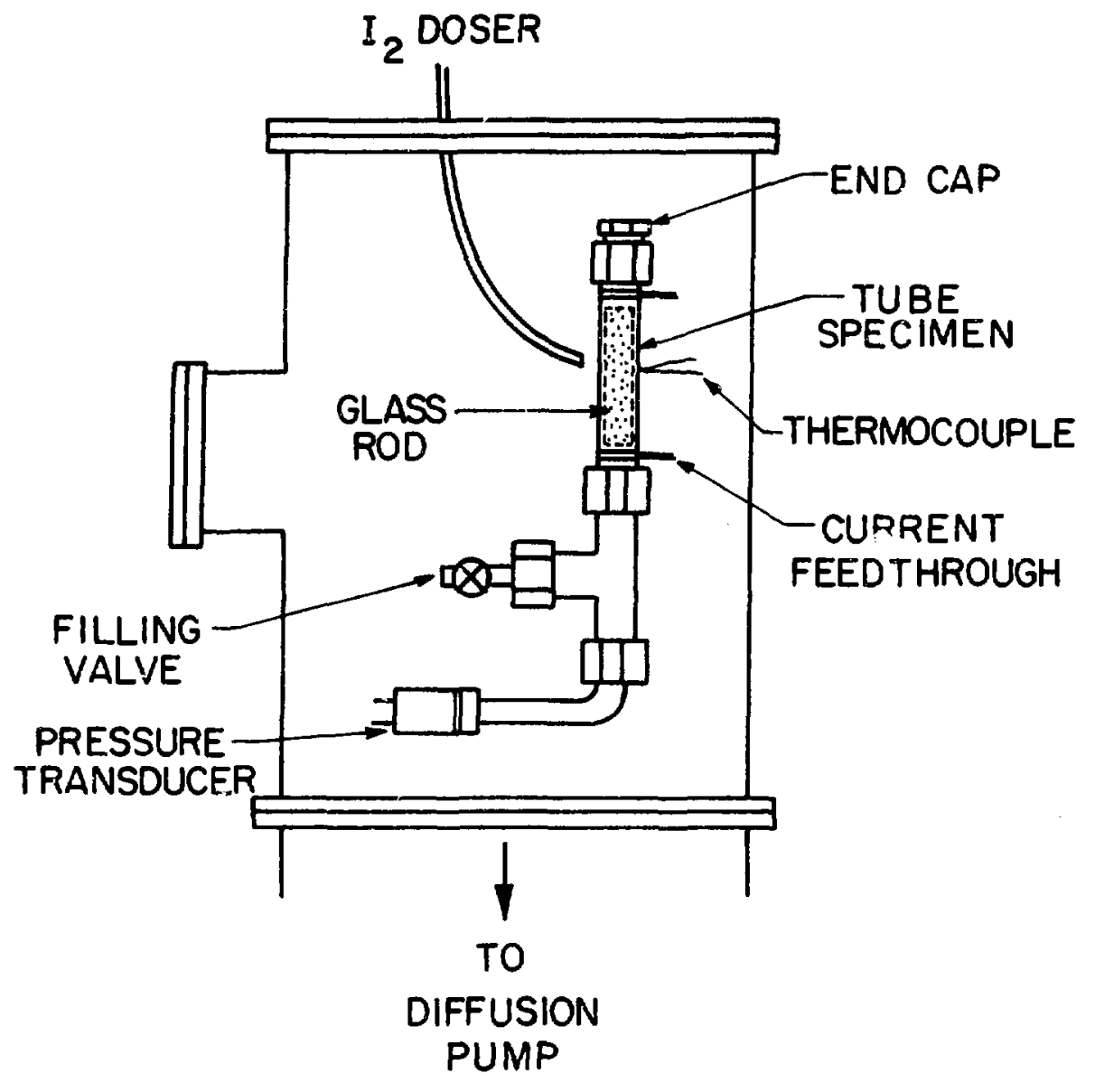

XBL 791-5528

Figure 1. Stress corrosion cracking apparatus. 


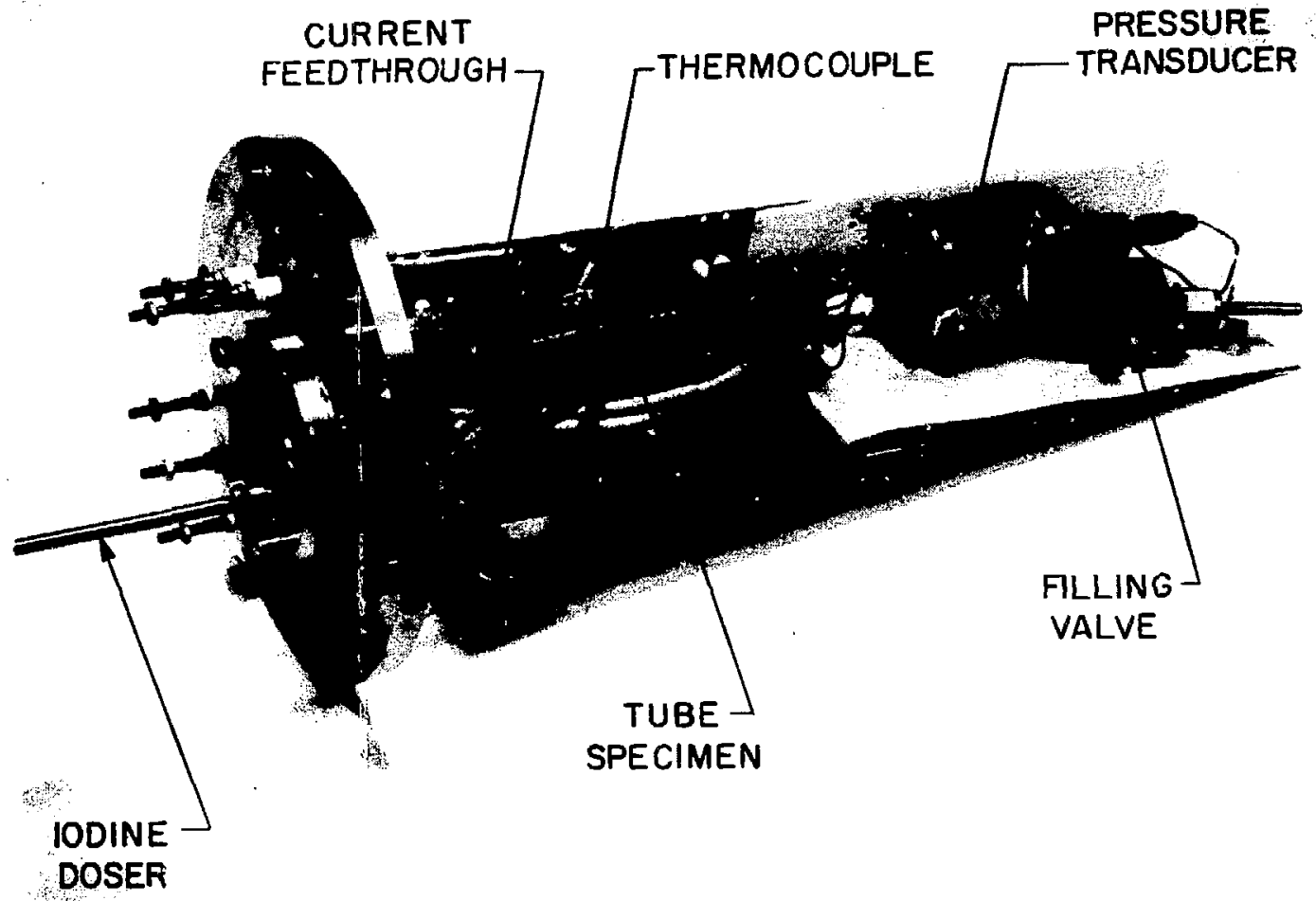



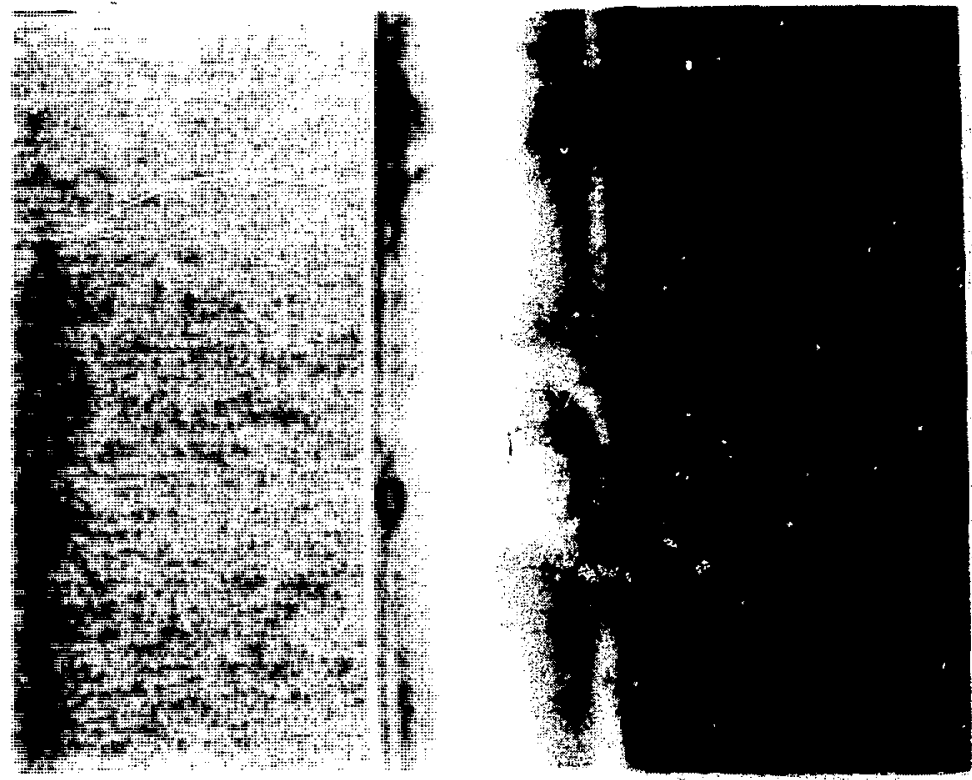

XBB791-473

Figure 2. Stress-relieved $\mathrm{Zx}-\mathrm{Z}$ (Sandvik lot no. 9AX32) failed after $13.17 \mathrm{hrs}$ under $45.8 \mathrm{Ksi}$ and 0.05 torx iodine at $300^{\circ} \mathrm{C}$. 


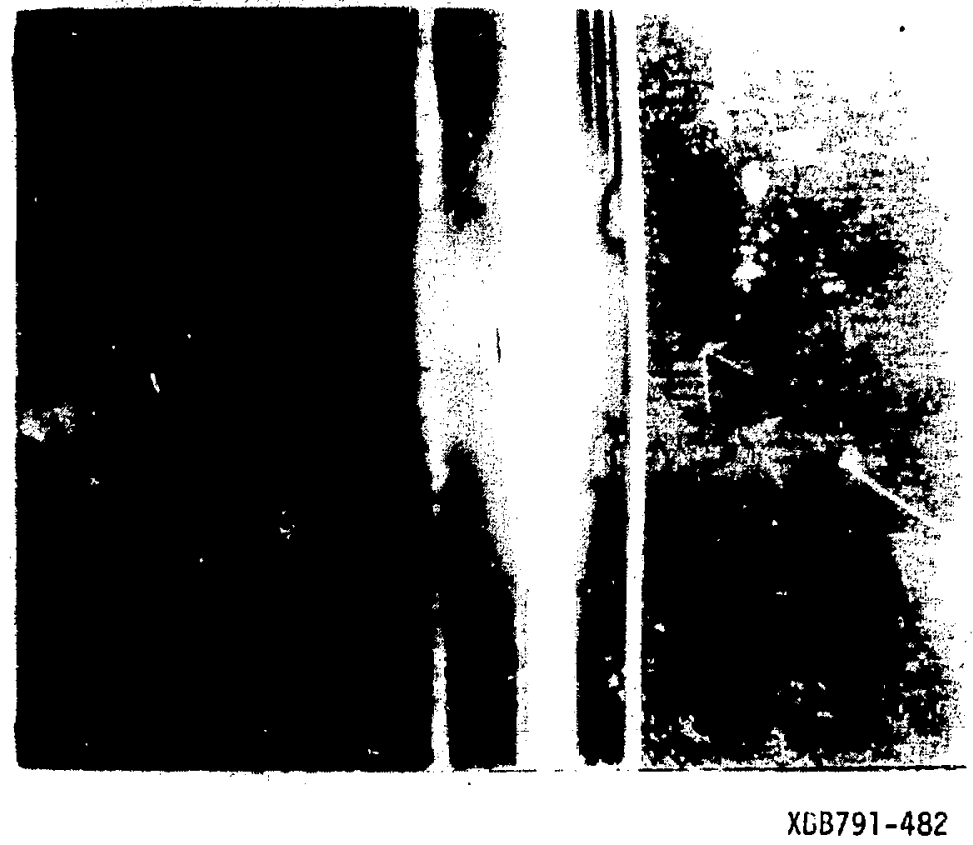

Figure 3. Stress-relieved $\mathrm{Zr}-2$ (Sandvik lot no. 9AX32) failed after $5.02 \mathrm{hrs}$ under $53.5 \mathrm{Ksi}$ and $0.05^{\circ}$ torr iodine at $300^{\circ} \mathrm{C}$. 


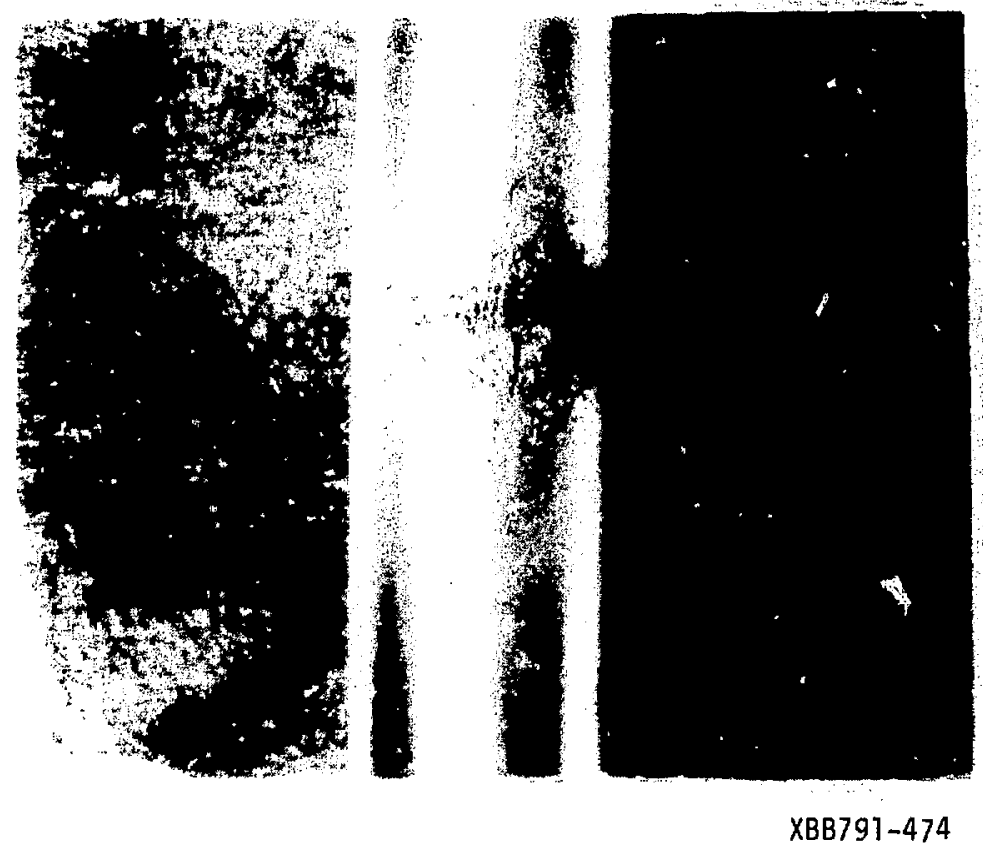

Figure 4. Stress-relieved $2 r-2$ (Sandvik lot no. 9Ax32) failed after 9.01 hrs under $53.5 \mathrm{ksi}$ and 0.032 torr iodine at $300^{\circ} \mathrm{C}$. 


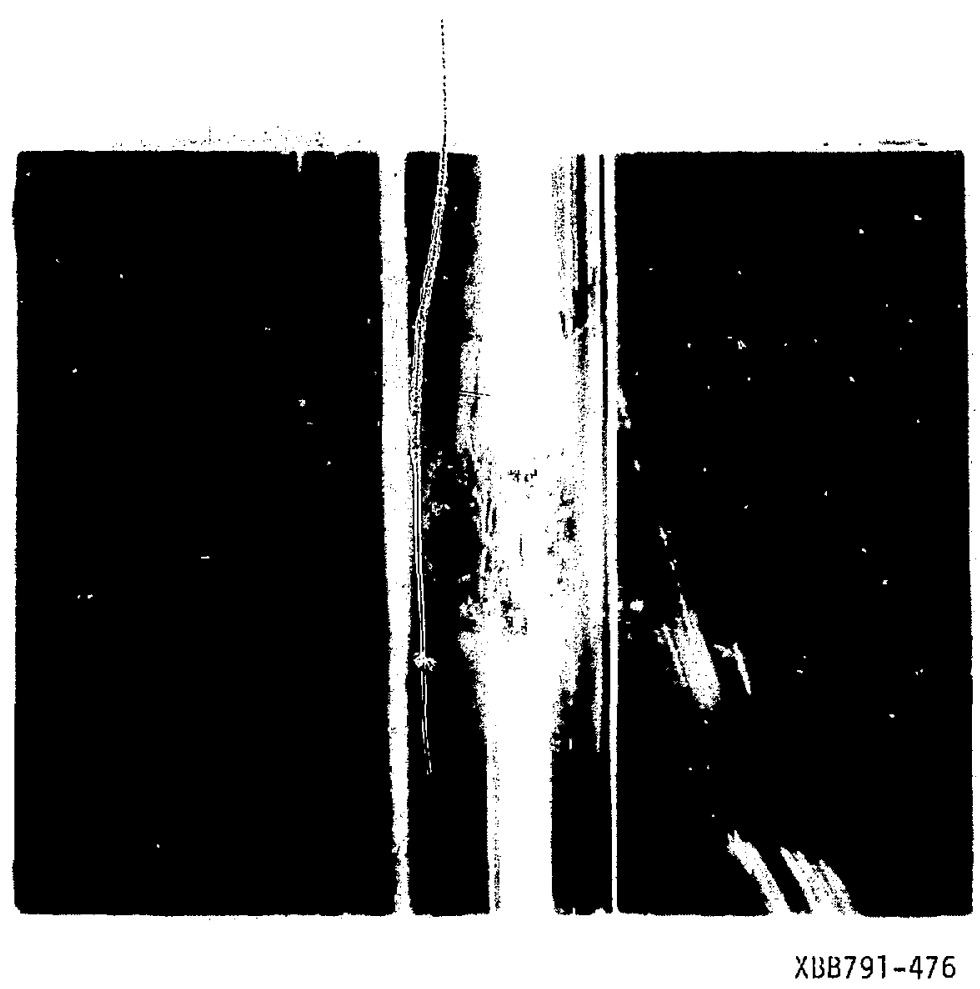

Figure 5. Stress-relieved $\mathrm{Zr-2}$ (Sandvik lot no. 9AX32) failed after $3.23 \mathrm{hrs}$ under $47.0 \mathrm{Ksi}$ and 0.16 torr iodine at $325^{\circ} \mathrm{C}$. 

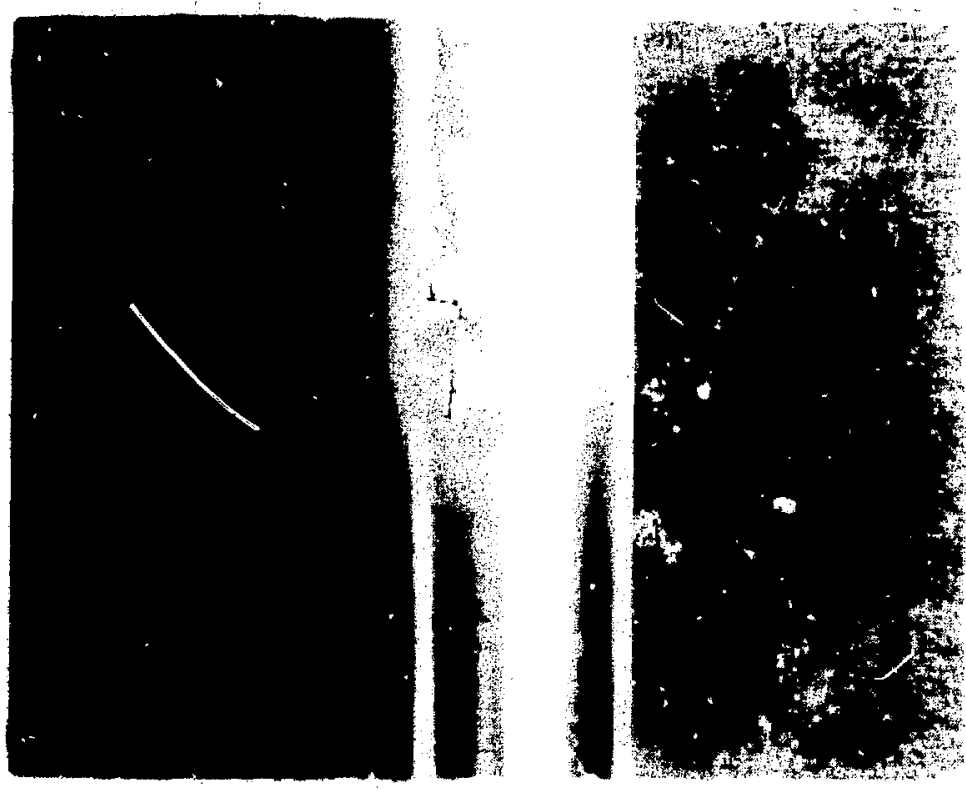

XBB791-481

Figure 6. Stress-relieved $\mathrm{Zr}-2$ (Sandvik lot no.9AX32) failed after 17.03 hrs under $45.1 \mathrm{Ksi}$ and 0.05 torr iodine at $270^{\circ} \mathrm{C}$. 


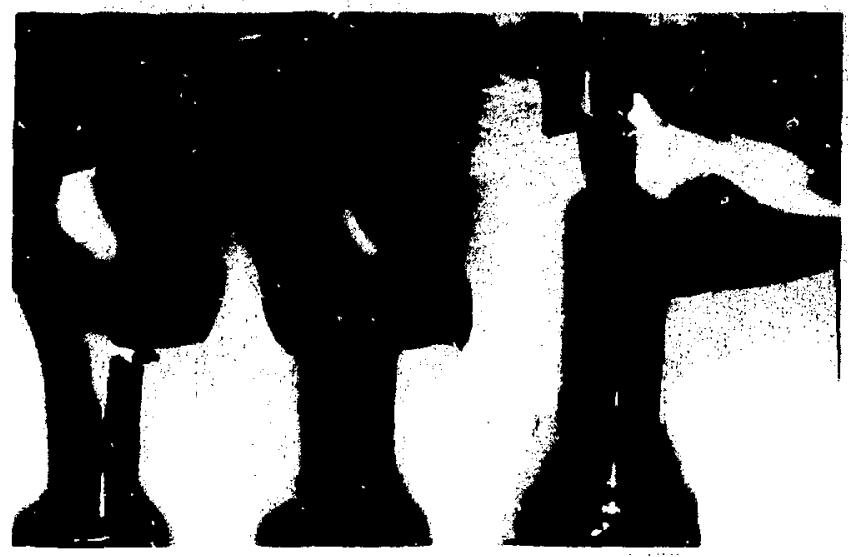

XBB 791-329A

Figure 7. Control specimens of Sandvik lot no. $9 A \times 32$ ruptured at $300^{\circ} \mathrm{C}$ without iodine at stresses of $55.5,53.6$, and $49.7 \mathrm{ksi}$. 


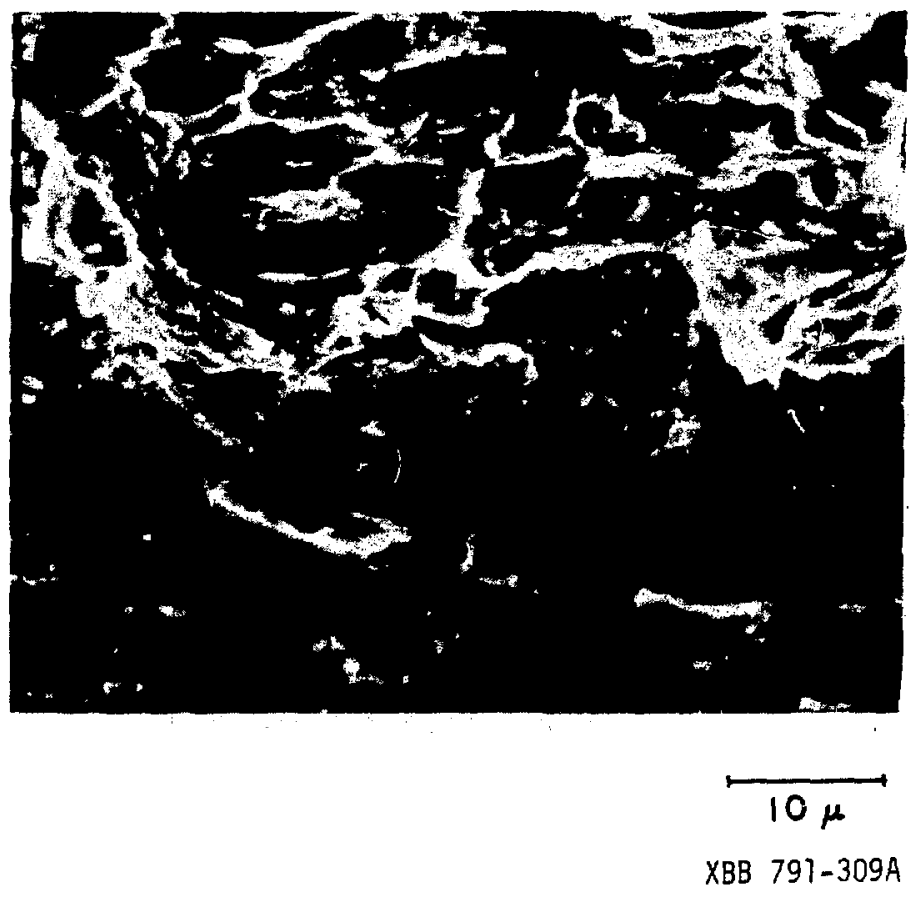

Figure 8. SEM fxactography of pin-hole type failure (preflawed stress relieved $\mathrm{zr}-2$ from SRI) failed after $20.5 \mathrm{hrs}, 52.3 \mathrm{ksi}$, 0.017 toxr iodine at $320^{\circ} \mathrm{C}$. 


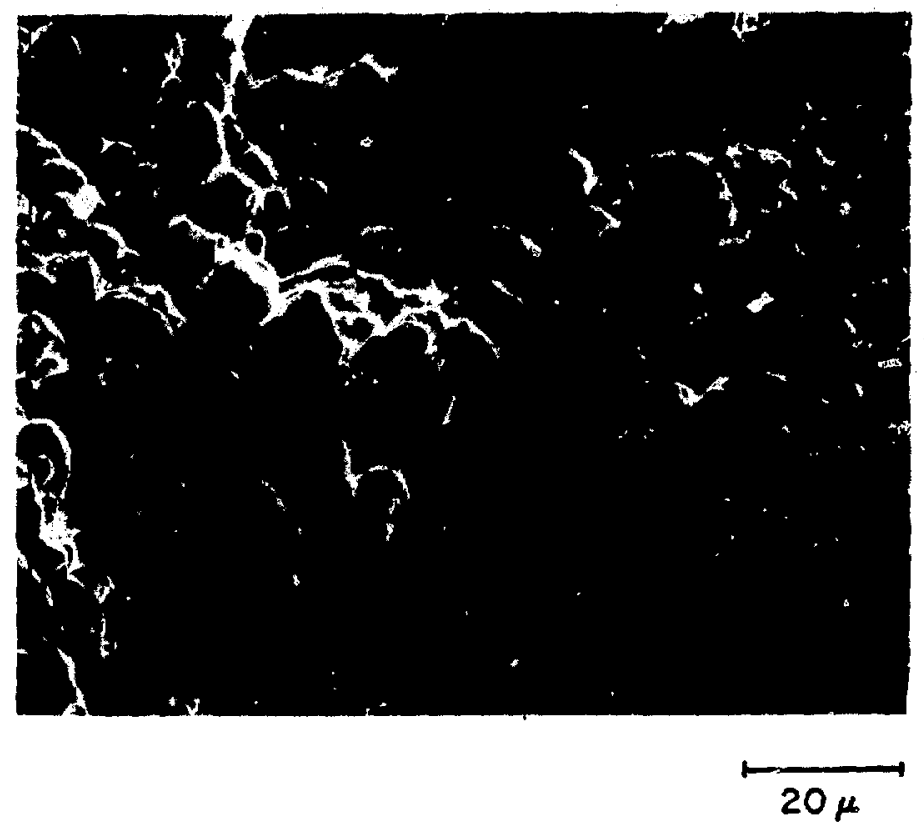

XBB 791-314A

Figure 9. SEM fxactography of ductile failure (unflawed, stressrelieved $\mathrm{zr}-2$ from SRI) failed after $51.8 \mathrm{hrs} ; 320^{\circ} \mathrm{C}$, without iodine. 


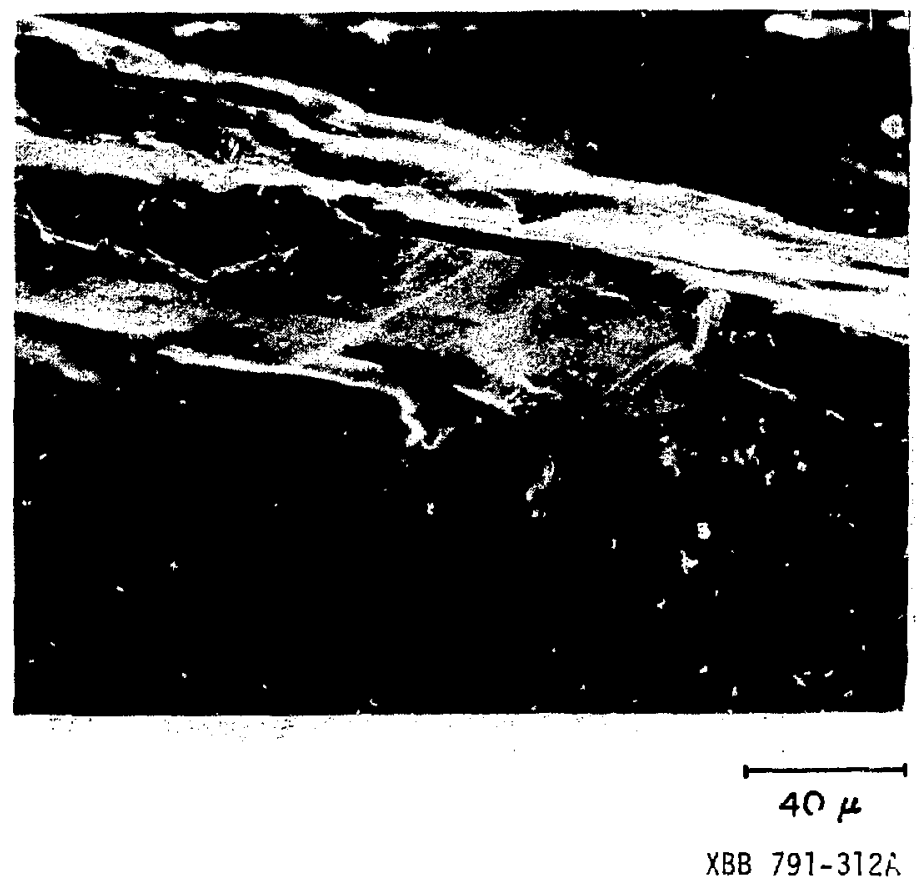

Figure 10. Besides main penetrating crack, there are alot of small cracks in iodine-affected area. (Preflawed stress-relieved Zr-2 fxom SRI failed after $59.3 \mathrm{hrs}, 52.3 \mathrm{ksi}, 0.045$ torr iodine, $320^{\circ} \mathrm{C}$.) 


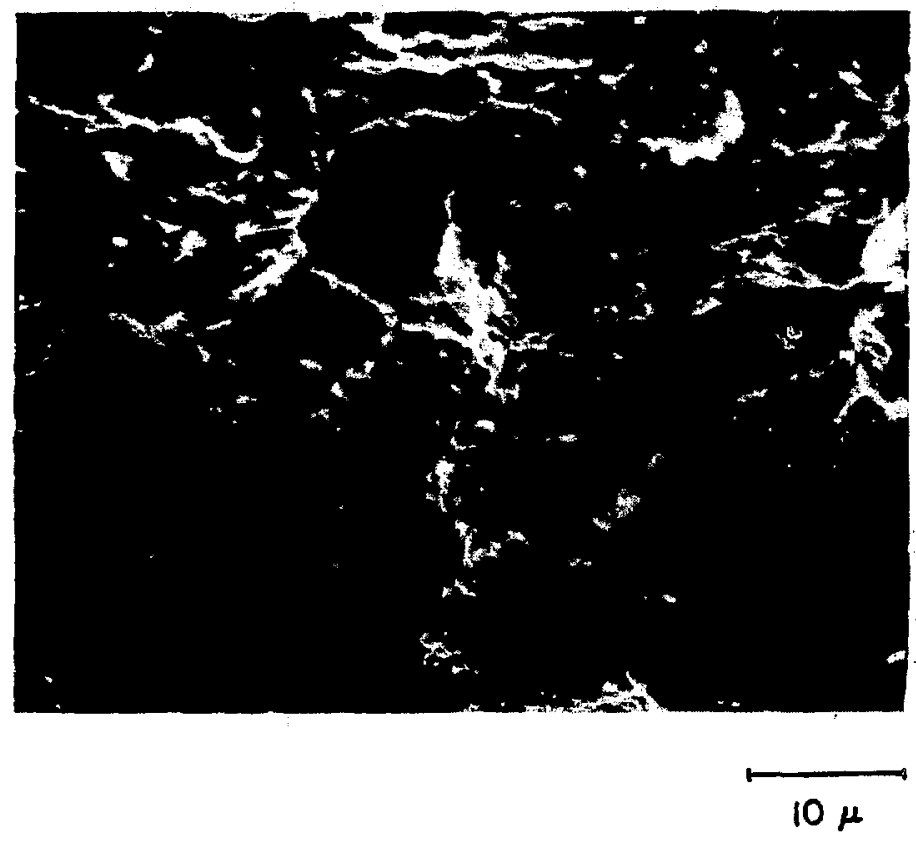

XBB 797-308A

Figure 11. Same specimen as Fig. 10 showing many small cracks branching out from main crack. 


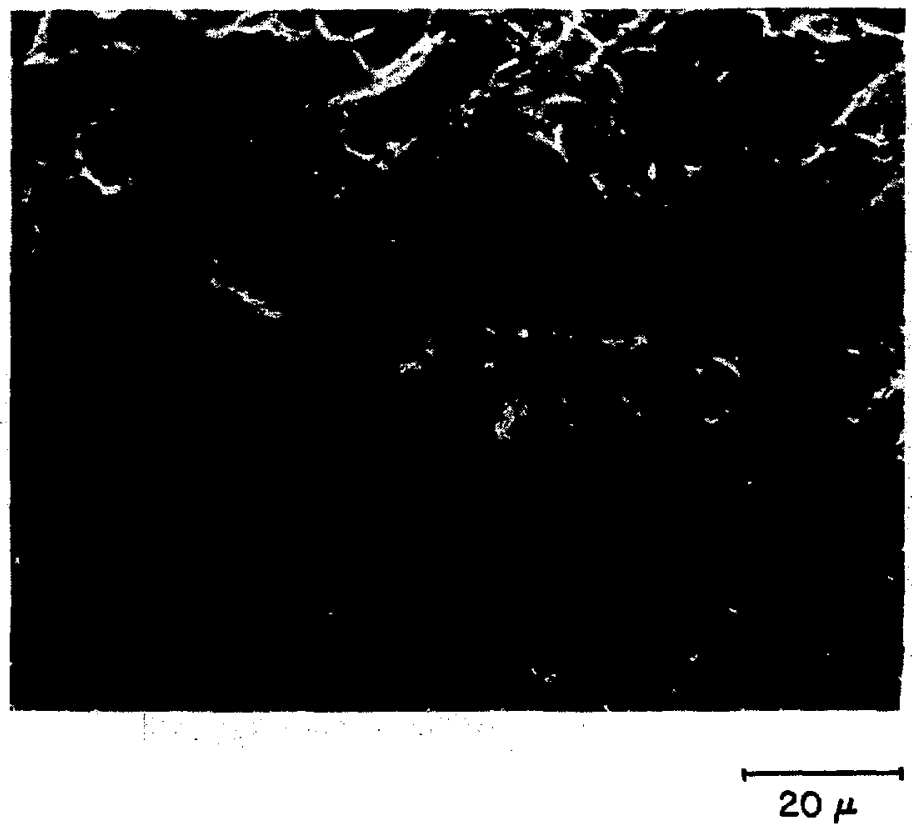

XBB 791-311A

Figure 12. Transition from brittle mode to ductile (dimple) mode. 


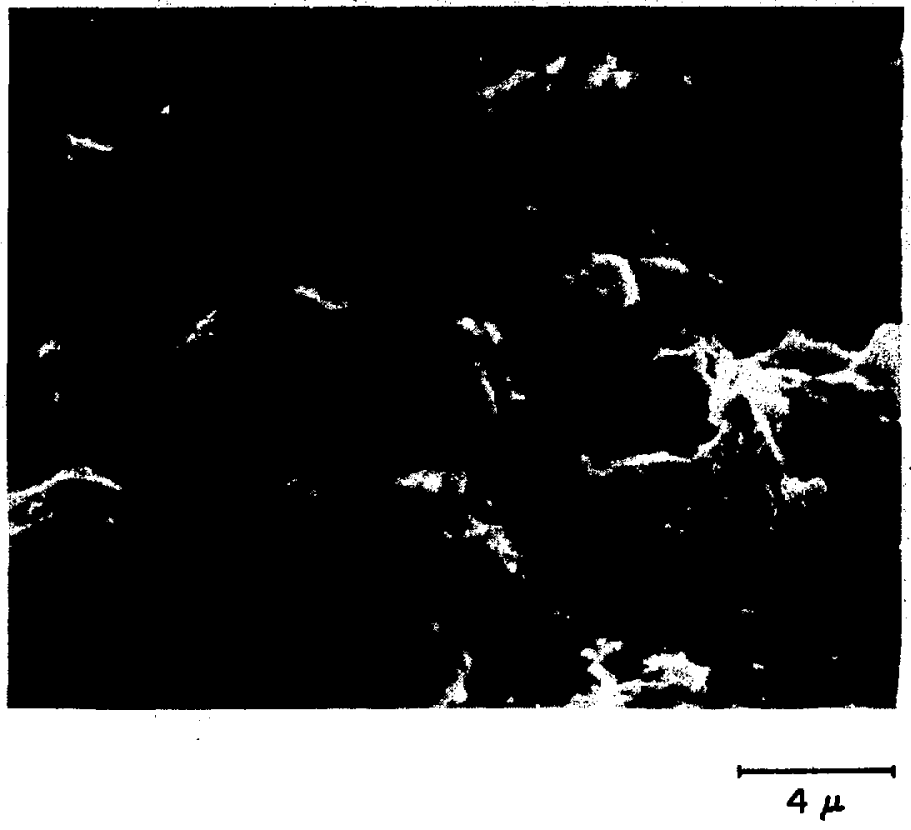

XBB 791-315A

Figure 13. Grain failed by cleavage (preflawed stress-relieved $\mathrm{Zr}-2$ from SRI) failed after $5.93 \mathrm{hrs} ; 52.3 \mathrm{Ksi}, 0.045$ torr iodine, $320^{\circ} \mathrm{C}$. 


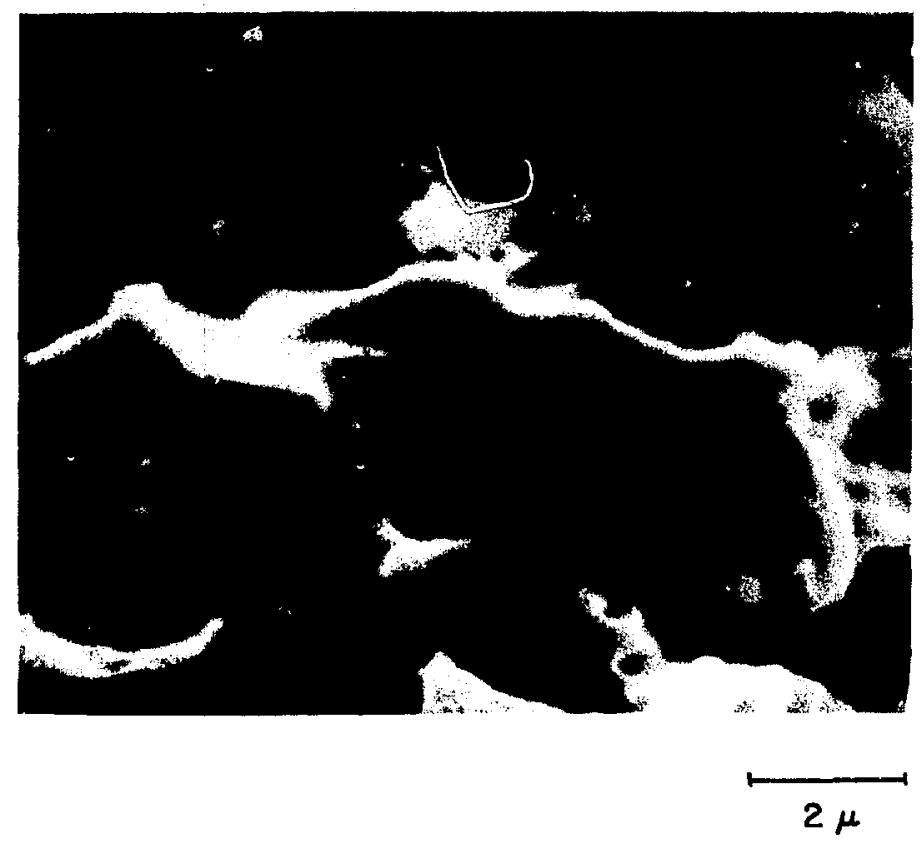

XBB $791-313 A$

Figure 14. Cleaved grain of Fig. 13 at higher magnification. 


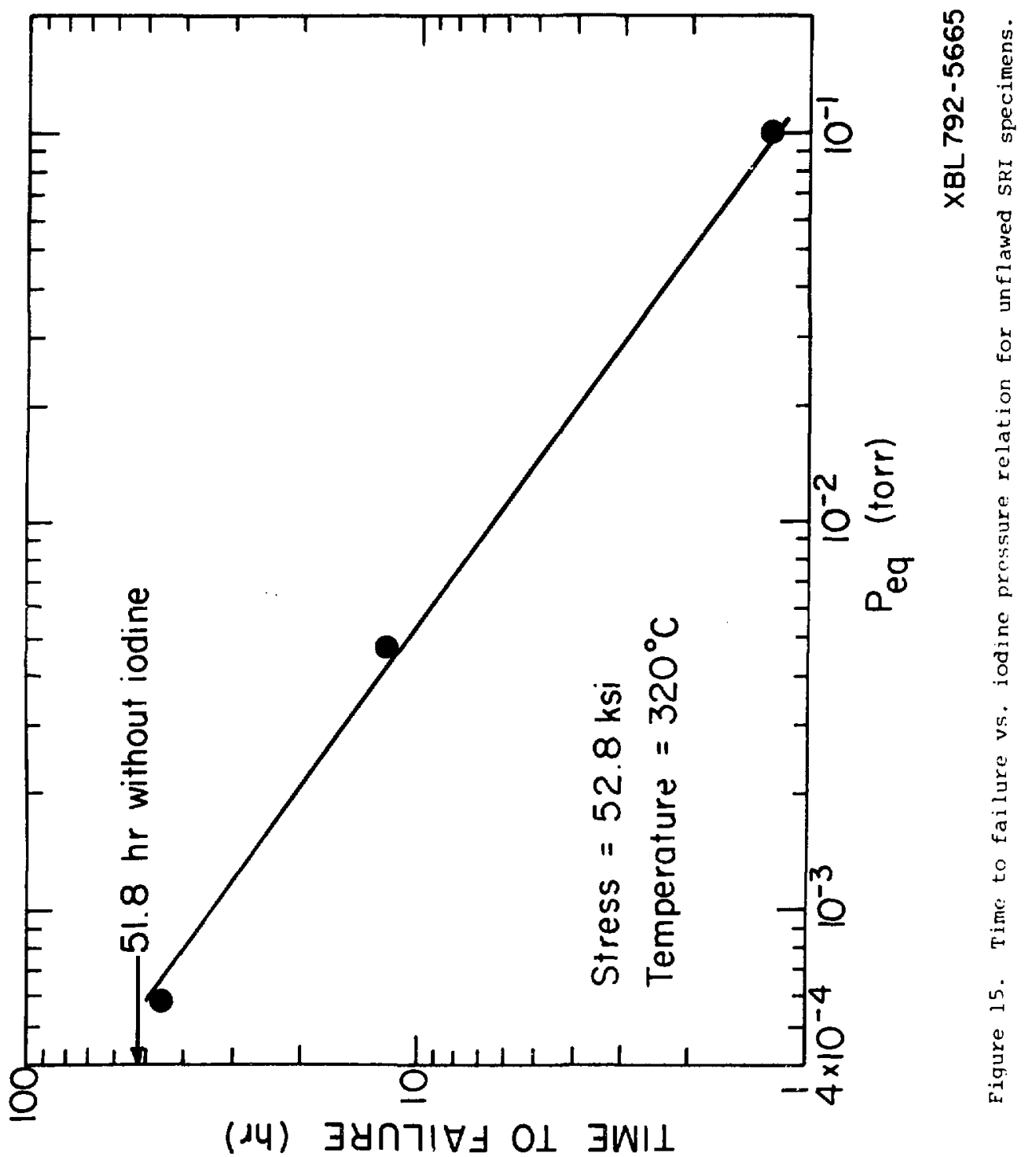




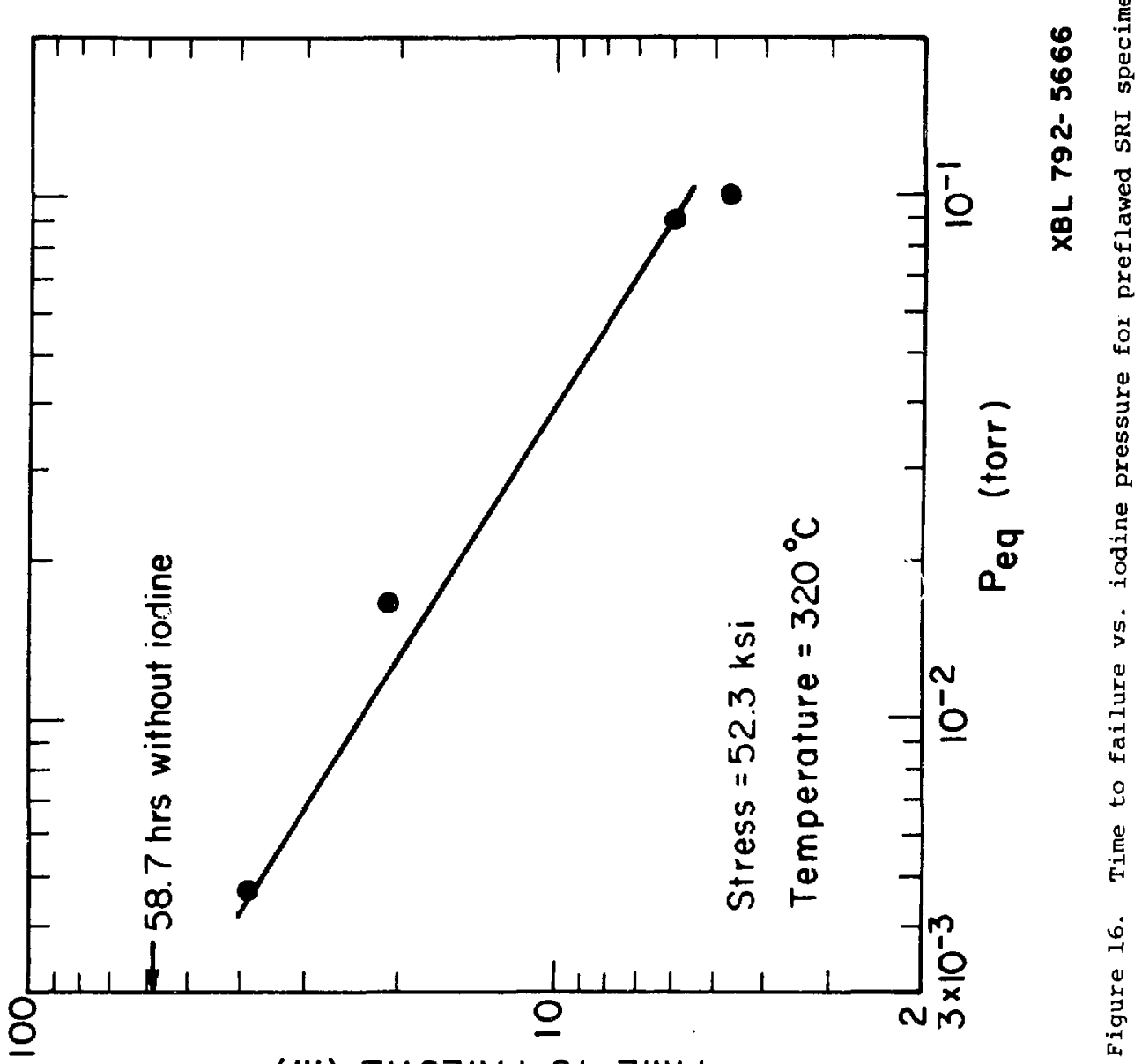

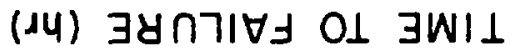




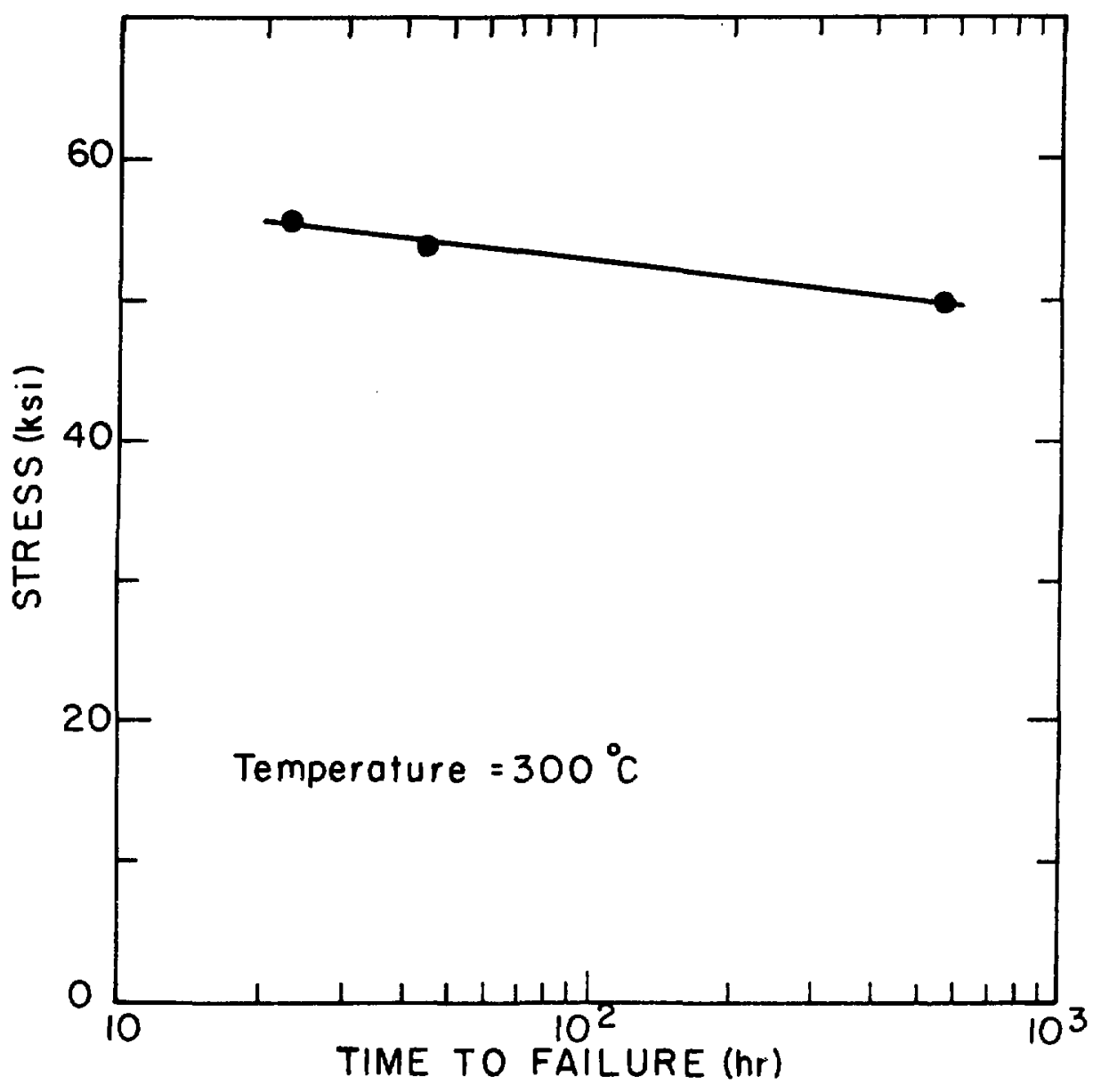

XBL7925667

Figure 17. Time to failure vs. stress for control specimens (Sandvik) at $300^{\circ} \mathrm{C}$ without iodine. 


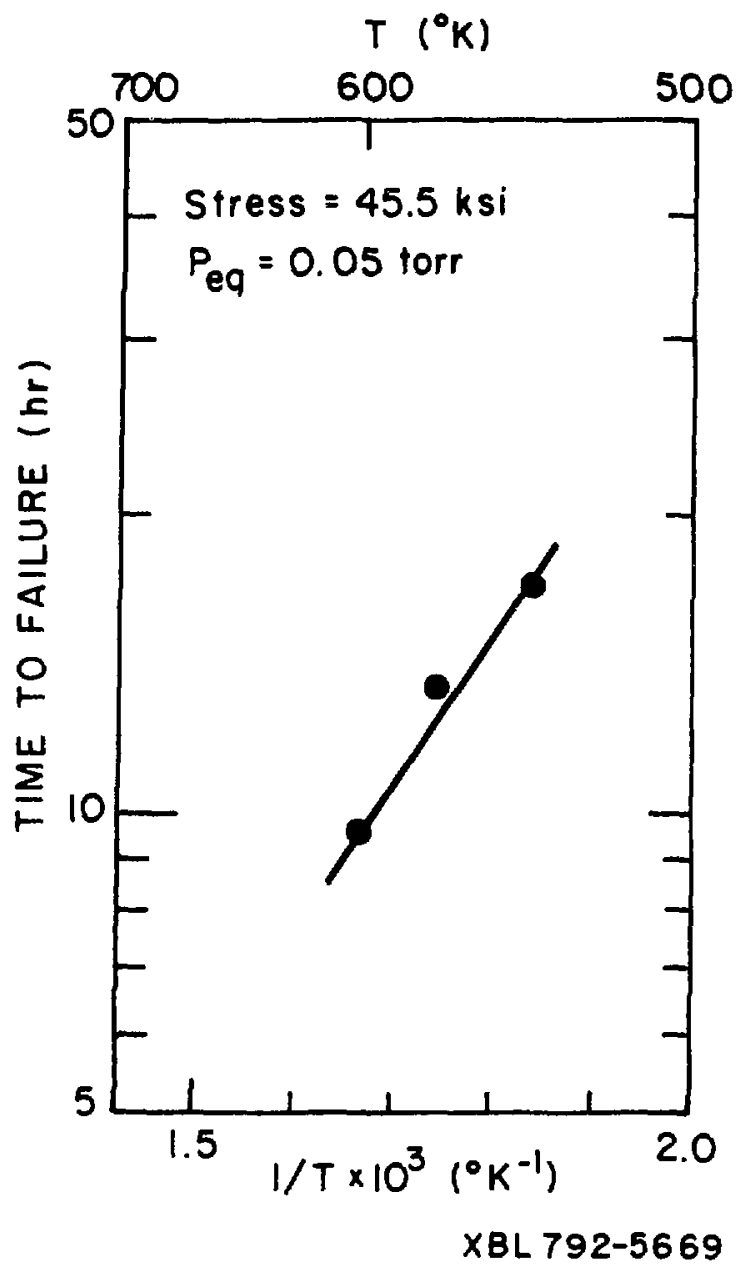

Figure 18. Time to failure vs. temperature for Sandvik specimens at $45.5 \mathrm{Ksi}$ and 0.05 torr iodine pressure. 


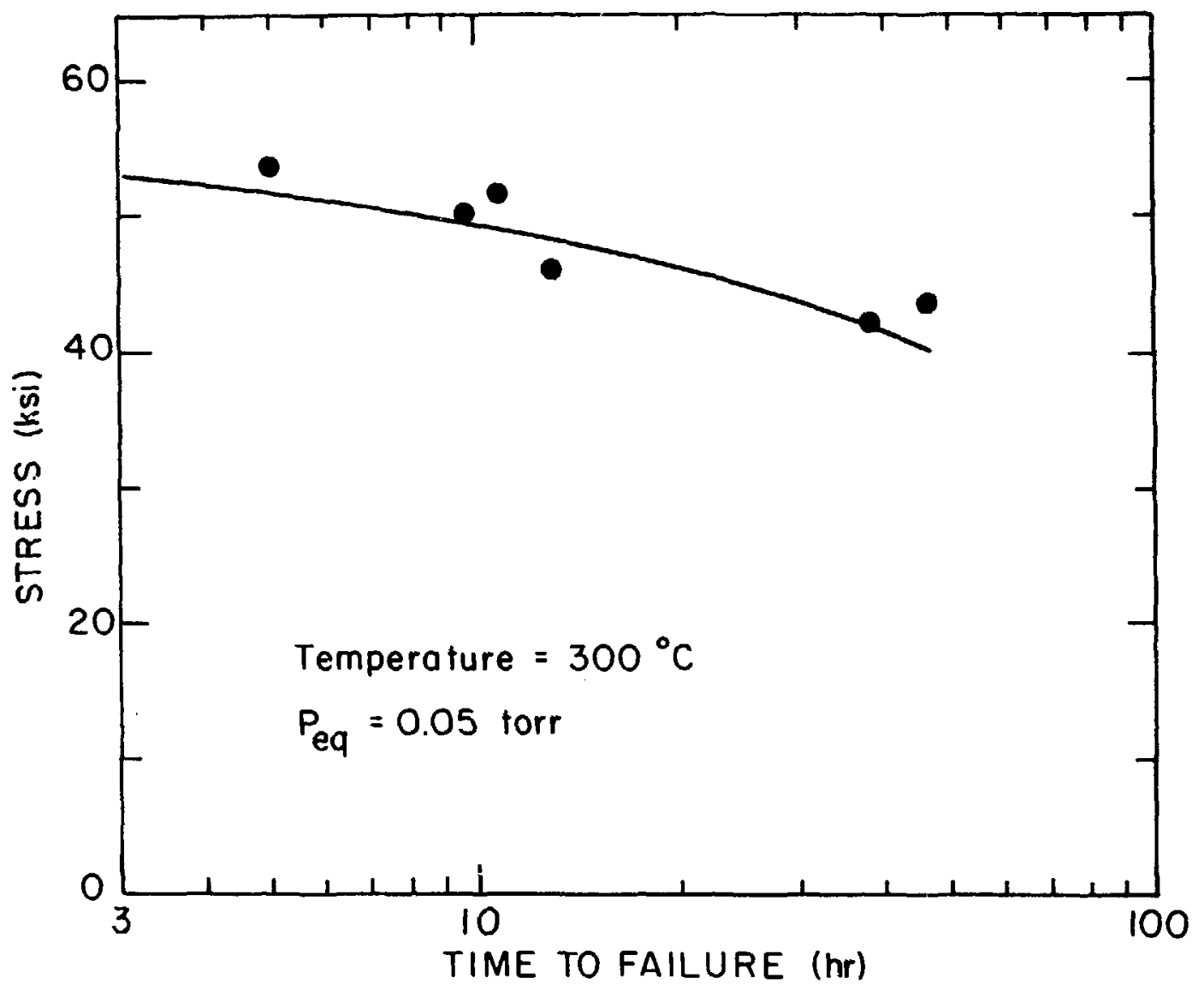

$x B 1792-5663$

Figure 19. Time to failure vs. stress for Sandvik specimens at $300^{\circ} \mathrm{C}$ and 0.05 torr iodine pressure. 


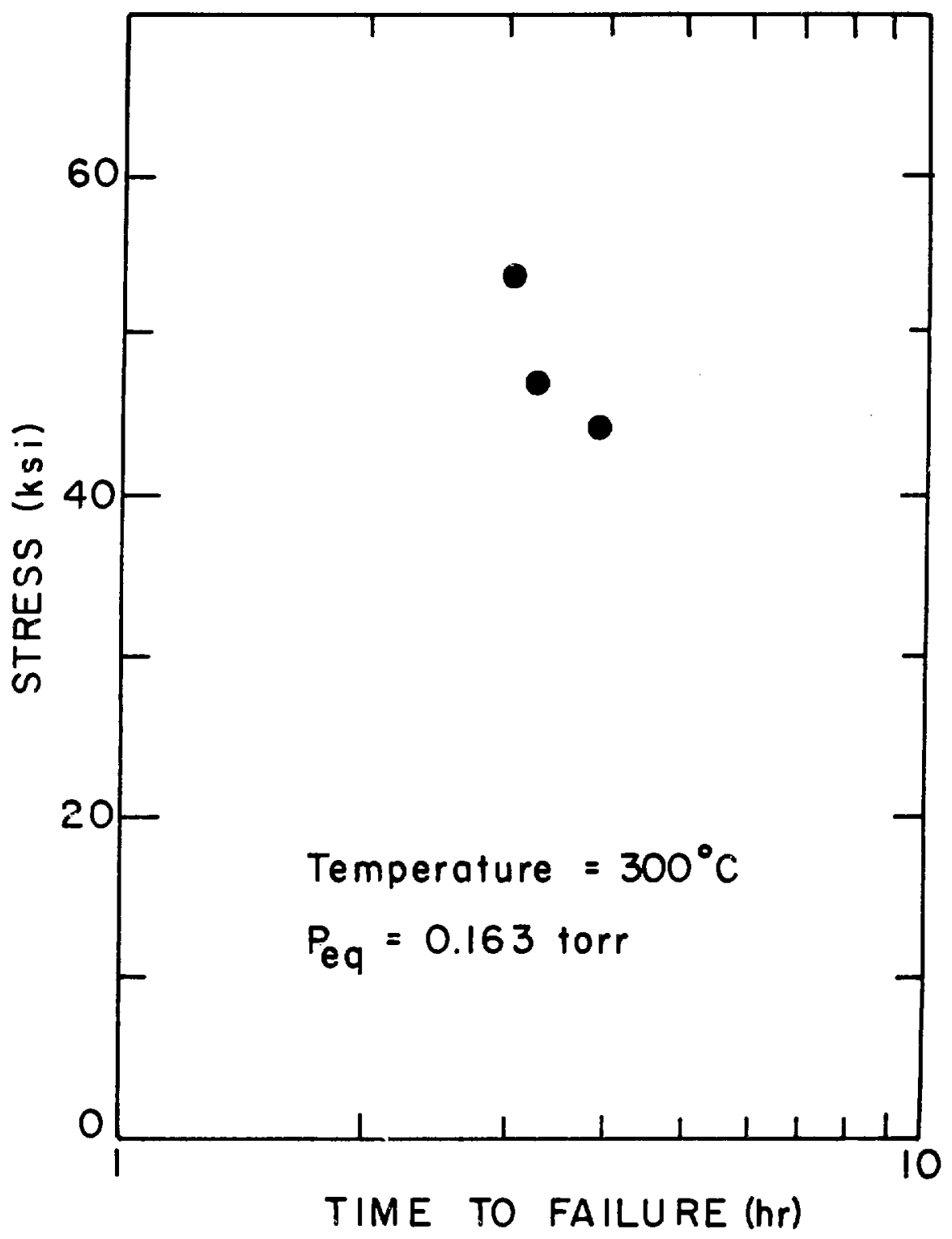

XBL $792-5664$

Figure 20. Time to failure vs. stress for Sandvik specimens at $300^{\circ} \mathrm{C}$ and 0.163 torr iodine pressure. 


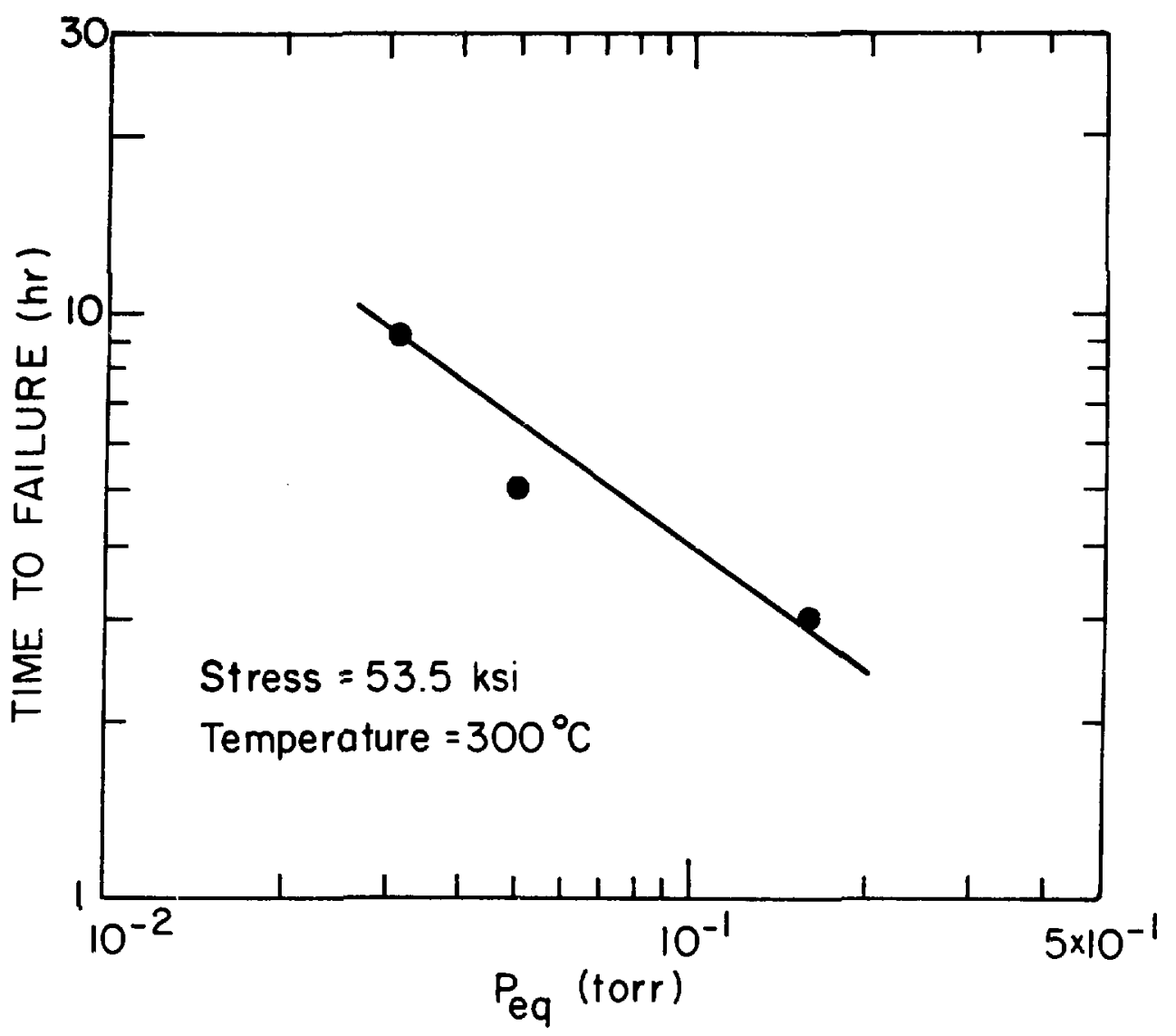

XBL792-5668

Figure 21. Time to failure vs. iodine pressure for Sandvik specimens at $53.5 \mathrm{Ksi}$ and $300^{\circ} \mathrm{C}\left(t_{F}=44.3 \mathrm{hrs}\right.$ for specimen without iodine). 
Reduction of $\mathrm{UO}_{2}$ and Measurement of the Oxygen-Metal Ratio by: Kee Kim

\section{Introduction}

Oxygen self-diffusion in hypostoichiometric uranium oxides is of importance in predicting the rate of oxyyen redistribution and other physicochemical processes occuring in an irradiatod fuel pin. Although there have been many measurements of oxygen diffusion in ordinary oxides and in hyperstoichiometric uranium dioxide $\left(\mathrm{UO}_{2+\mathrm{x}}\right)(1)$, similar measurements in $\mathrm{UO}_{2-x}$ have been proven very difficult mainly berause $\mathrm{UO}_{2-x}$ is a defect structure stable only at $\mathrm{T}>1300^{\circ} \mathrm{C}$, and its oxygen diffusivity is likely to be large enough to render convertional methods unworkable (i.e.. gas phase mass transfer of surface isotope exchange are rate limiting). The method proposed involves a diffusion couple consisting of $\mathrm{UO}_{2-\mathrm{x}}^{18}$ wafer sandwscled to a $10_{2-x}^{16}$ water by a bond of liquid uranium. When heated, the $0^{18}$ and $0^{16}$ will interdiffuse. The liquid uranium at the interface is intended to eliminate a gap resistance to oxygen transfer in a manner similar to a sodium bond in carbide LMFBR fucl pin. The hypostoichiometric samples are to be prepared by hydrogen reduction of $\mathrm{UO}_{2}$ at high temperature:

$$
\mathrm{UO}_{2}+\mathrm{xH}_{2}=\mathrm{UO}_{2-\mathrm{x}}+\mathrm{xH}_{2} \mathrm{O}
$$

The course of the reduction process will be followed by an electromicrobalance which continuously monitors the weight of sample. Betore each diffusion test the stoichiometry of the sample should be determined.

\section{Theory of the $c / U$ Determination}

Since there is a substantial amount of evaporation at high temperature the stoichiometry can not be measured directly by the total weight change during the reduction step. This problem can be overcome simply by having another ficce of urania present during the reduction step and subsequently oxidizing this test specimen back to $\mathrm{UO}_{2}$ at lower temperature while measuring the weight gain, from which the stoichiometry can be calculated by: 


$$
O / U=2.0-\frac{270}{16} \frac{d w}{w}
$$

where $d w=$ weight gain and $w=$ specimen weight. The oxidation of $\mathrm{VO}_{2-\mathrm{x}}$ can be achieved by flowing gas which has the equilibrium oxygen potential of stoichiometric $\mathrm{NO}_{2}$. The equilibrium oxygen potential of $\mathrm{vO}_{2+\mathrm{x}}, \overline{\Lambda G}_{\mathrm{O} 2^{\prime}}$ is well known as a function of temperature and stoichiometry and is shown in Fig. $1(2,3)$. As can be seen in fig. 1 , the oxygen potential changes sharply in the vicinity of stoichiometric $\mathrm{VO}_{2}$. By using known ratios of $\mathrm{H}_{2} \mathrm{O}$ and $\mathrm{H}_{2}$ and controlling the temperature, the desired oxygen potential can be obtained as follows:

$$
\mathrm{H}_{2}+\frac{1}{2} \mathrm{O}_{2} \Rightarrow \mathrm{H}_{2} \mathrm{O}(\mathrm{g})
$$

If $\wedge \mathrm{G}_{\mathrm{H}}^{\circ}{ }_{2} \mathrm{O}$ is the standard free energy of formation of $\mathrm{H}_{2} \mathrm{O}(\mathrm{g})$, have:

$$
\mathrm{k}=\frac{\mathrm{P}_{\mathrm{H}_{2} \mathrm{O}}}{\mathrm{P}_{\mathrm{H}_{2}{ }^{\mathrm{P}} \mathrm{O}_{2}} 1 / 2}=\exp \left[-\Delta \mathrm{G}_{\mathrm{H}_{2} \mathrm{O}}^{\mathrm{O}} / \mathrm{RT}\right]
$$

For gas-solid equilibrium the oxygen potential of the fuel must be equal to that of the $\mathrm{H}_{2} / \mathrm{H}_{2} \mathrm{O}$ gas rnixture or:

$$
\overline{\triangle G}_{\mathrm{O}_{2}}=\mathrm{RT} \operatorname{lnP} \mathrm{O}_{2}=2 \mathrm{RT} \ln \frac{\mathrm{P}_{2} \mathrm{O}}{\mathrm{P}_{\mathrm{H}_{2}}}+2 \Delta \mathrm{G}_{\mathrm{H}_{2} \mathrm{O}}
$$

Using this relationship, the ordinate of the fig. 1 can be converted to the $\mathrm{H}_{2} \mathrm{O} / \mathrm{H}_{2}$ ratio, as is shown in $\mathrm{Fig}$. 2. According to this graph, at $1550 \mathrm{C}$ stoichiometric $\mathrm{UO}_{2}$ is obtained in hydrogen-water mixtures ranging from $\mathrm{H}_{2} \mathrm{O} / \mathrm{H}_{2}=10^{-7}$ to $\mathrm{H}_{2} \mathrm{O} / \mathrm{H}_{2}=10^{-1}$. The desired $\mathrm{H}_{2} \mathrm{O} / \mathrm{H}_{2}$ ratio is obtained by bubbling hydrogen through water and by controlling the temperature of water. The hydrogen does lot have to be saturated by water because of the extremely wide range of oxygen potential of $\mathrm{UO}_{2}$. In this experiment the water was kept at $7^{\circ} \mathrm{C}$, which gives $\mathrm{H}_{2} \mathrm{O} / \mathrm{H}_{2}$ ratio of $10^{-2}$ or less. 


\section{Experimental}

Fig. 3 shows the setup of the furance. The samples are suspended from the electromicrobalance by a rhenium wire. The gas is fea into the bottom of the molybdenum crucible and flows upward. The crucible is heated by a tungsten mesh heating element from the outside. The temperature is masured by a tungsten-rhenium thermocouple located inside the crucible. The $\mathrm{UO}_{2}$ wafers are $0.5-1 \mathrm{~mm}$ thick and $1.2 \mathrm{~cm}$ diameter. Fig. 4 is a flow diagram of the system. Hydrogen is purified by charcoal filled liquid nitrogen trap. Gas is exhausted into the air through diffusion pump oil to prevent back diffusion of air into the crucible. Samples are reduced in pure hydrogen at a flow rate of $29 \mathrm{cc} / \mathrm{sec}$ at $1950^{\circ} \mathrm{C}$ and $15 \mathrm{cc} / \mathrm{sec}$ at $1850^{\circ} \mathrm{C}$. Before oxidizing the reduced samples, its weight is measured by Mettler microbalance. Then it is placed in a rhenium basket suspended from the electromicrobalance by a rhenium wire. The reduced sampless aro oxidized to $\mathrm{UO}_{2}$ using hydrogen stream of $\mathrm{H}_{2} \mathrm{O} / \mathrm{H}_{2} \leq 10^{-2}$ at temperature range of $1200-1400^{\circ} \mathrm{C}$. The experimental procedures is:

1. Pump out the whole system and degas the sample at $200^{\circ} \mathrm{C}$.

2. Cool down to room temperature and fill the system with helium.

3. Heat the sample to $800^{\circ} \mathrm{C}$ and record the electromicrobalance weight of the sample in this condition.

4. Flow hydrogen at $10 \mathrm{cc} / \mathrm{sec}$. (Valves 1 and 2 in fig 4 closed; valves $\hat{3}$ and 4 open).

5. In flowing $\mathrm{H}_{2}$, heat the sample up to $1300^{\circ} \mathrm{C}$.

6. Flow $\mathrm{H}_{2} \mathrm{O} / \mathrm{H}_{2}$ the mixture by opening valves 1 and 2 and closing valves 3 and 4 until the weight reaches steady stato.

7. Flow dry hydrogen.

8. Cool down to $800^{\circ} \mathrm{C}$.

9. Flush the system with helium and record the electromicrobalance weight.

10. Cool down to room temperature. 
11. Take out the sample and measure the final weight using the Mettler balance.

The weight measurements of step 3 and 9 give the weight increases. The measurement of the initial and final weights by the Mettler microbalance also gives the weight increasment to be compared with the value obtained by electrumicrobalance in septs 3 and 9 . In order to make sure that the rhenium wire and basket do not react with $\mathrm{H}_{2}{\mathrm{O}-\mathrm{H}_{2}}_{2}$ in the operating condition, a dummy test was done without any sample in the basket. This test showed no weight changes through $1500^{\circ} \mathrm{C}$, so that all the weight increase is attributed to oxidation of the sample.

IV. Results

\begin{tabular}{|c|c|c|c|c|c|c|}
\hline \multicolumn{3}{|c|}{ Reducing Concitions } & \multicolumn{4}{|c|}{ Oxidizing back to $\mathrm{UO}_{2.0}$} \\
\hline Temp, ${ }^{\circ} \mathrm{C}$ & $\begin{array}{c}\mathrm{H}_{2}(\mathrm{std}) \mathrm{cc} / \mathrm{sec} \\
\text { flow rate }\end{array}$ & $\begin{array}{c}\text { time, } \\
\text { hrs }\end{array}$ & $w_{i}(g)$ & $d w w_{1}(m g)$ & $d w_{2}(m g)$ & $0 / U$ \\
\hline $1850^{\circ} \mathrm{C}$ & 20 & 1 & 0.6039 & - & 0.99 & 1.972 \\
\hline $1850^{\circ} \mathrm{C}$ & 10 & 1 & 0.5728 & -- & 1.00 & 1.970 \\
\hline $1850^{\circ} \mathrm{C}$ & 20 & 2 & 0.5702 & -- & 1.57 & .1 .954 \\
\hline $1950^{\circ} \mathrm{C}$ & 20 & 2 & 1.1532 & 2.651 & 2.65 & 1.951 \\
\hline $1950^{\circ} \mathrm{C}$ & 20 & 4 & 1. 1886 & - & 3.20 & 1.955 \\
\hline $1950^{\circ} \mathrm{C}$ & 20 & 4 & 1.2080 & 3.298 & 3.30 & 1.954 \\
\hline
\end{tabular}




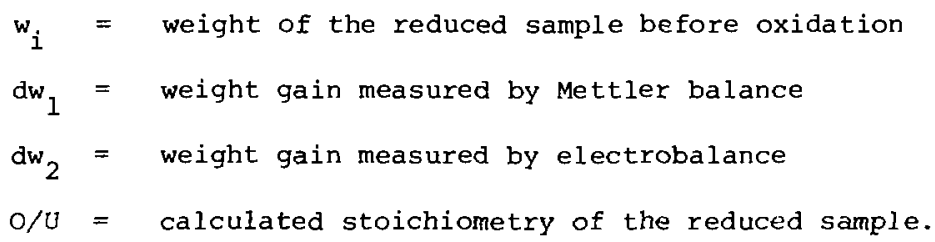

For example, the first row is for a sample which was reduced for 1 hr at $1850^{\circ} \mathrm{C}$ and $\mathrm{H}_{2}$ flow rate of $20 \mathrm{cc} / \mathrm{sec}$. The reduced sample weight is $0.6039 \mathrm{~g}$, which increased by $0.99 \mathrm{mg}$ when oxidized to $\mathrm{vO}_{2}$. The stoichiometry was calculated from Eq (1) to be 1.972. Precision of $0 / \mathrm{U}$ values is \pm 0.007 . As can be seen the reduction rates rapidly slow down with time (i.e. for the $4 \mathrm{hr}$ run, more than half of the redu: tion was achieved in the first 1 hr.)

\section{Future Experiments}

In order to make the $\mathrm{vo}_{2-\mathrm{x}}^{18}$ wafer which is part of the diffusion couple, $\mathrm{O}^{16}$ in a $\mathrm{UO}_{2}^{16}$ wafer will be substituted by $\mathrm{O}^{18}$ using $\mathrm{H}_{2} \mathrm{O}^{18}-\mathrm{H}_{2}$ mixtures using the same method is employed in oxidation of $\mathrm{vO}_{2-x}$ (i.e. flow $\mathrm{H}_{2}$ thru $\mathrm{H}_{2} \mathrm{O}^{18}$ and expose $\mathrm{UO}_{2-\mathrm{x}}^{16}$ to this stream at $1200^{\circ} \mathrm{C}-1500^{\circ} \mathrm{C}$ ). One wafer of $\mathrm{vo}_{2}^{16}$ and one of $\mathrm{uO}_{2}^{18}$ will be reduced to $\mathrm{vo}_{2-\mathrm{x}}^{16}$ and $\mathrm{vo}_{2-\mathrm{x}}^{16}$ respectively. and the diffusion couple will be fabricated.

\section{References}

1. J.F. Marin and P. Contamin, J. Nucl. Mater. 30, 16 (1969)

2. N.A. Javed, J. Nucl. Mater. 43, 219 (1972)

3. P.E. Blackburn, J. Nucl. Mater. 46, 244 (1973) 


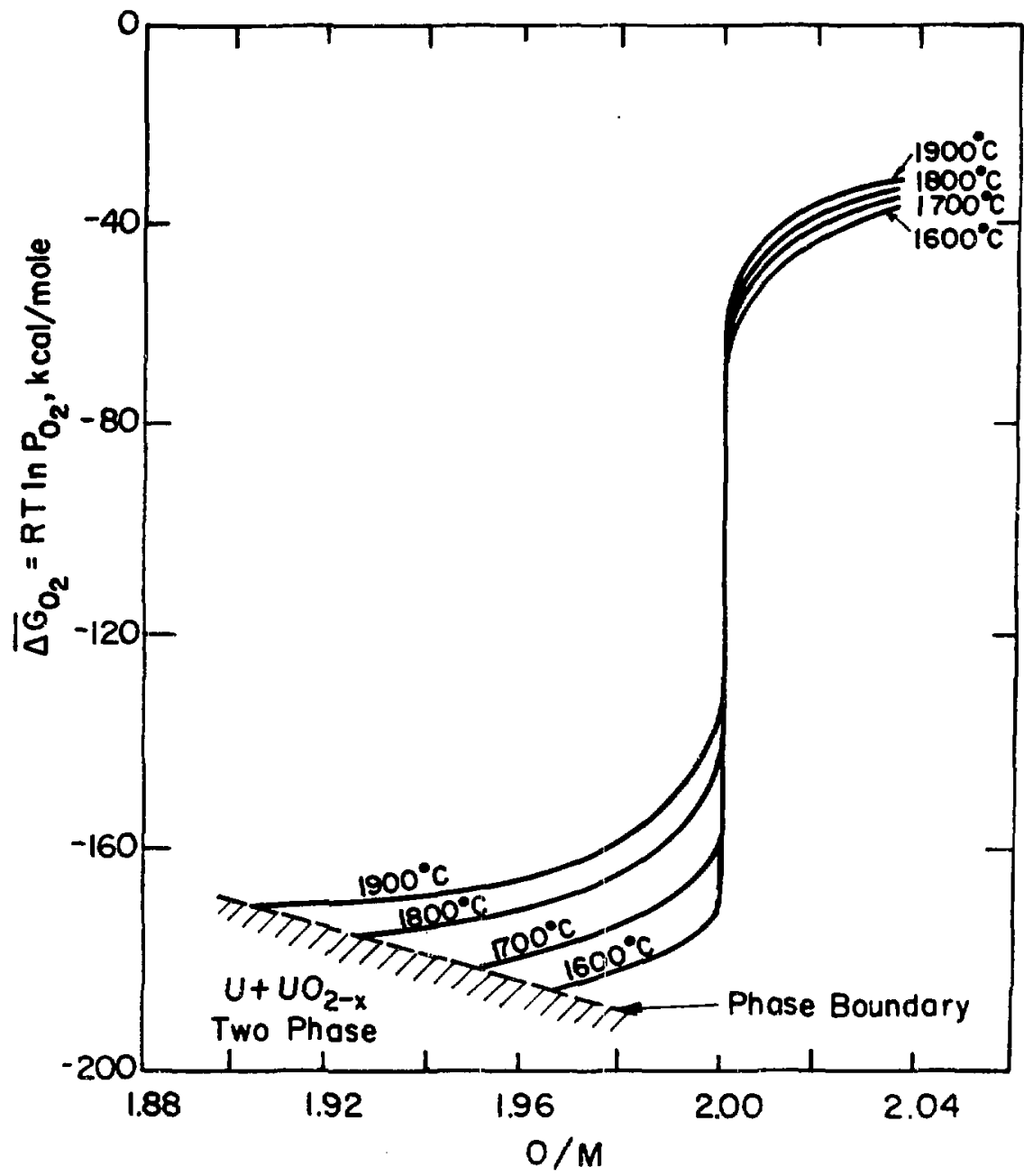

XBL $192-5670$

Figure 1. Variation of oxygen potential with temperature and $O / M$ ratio. 


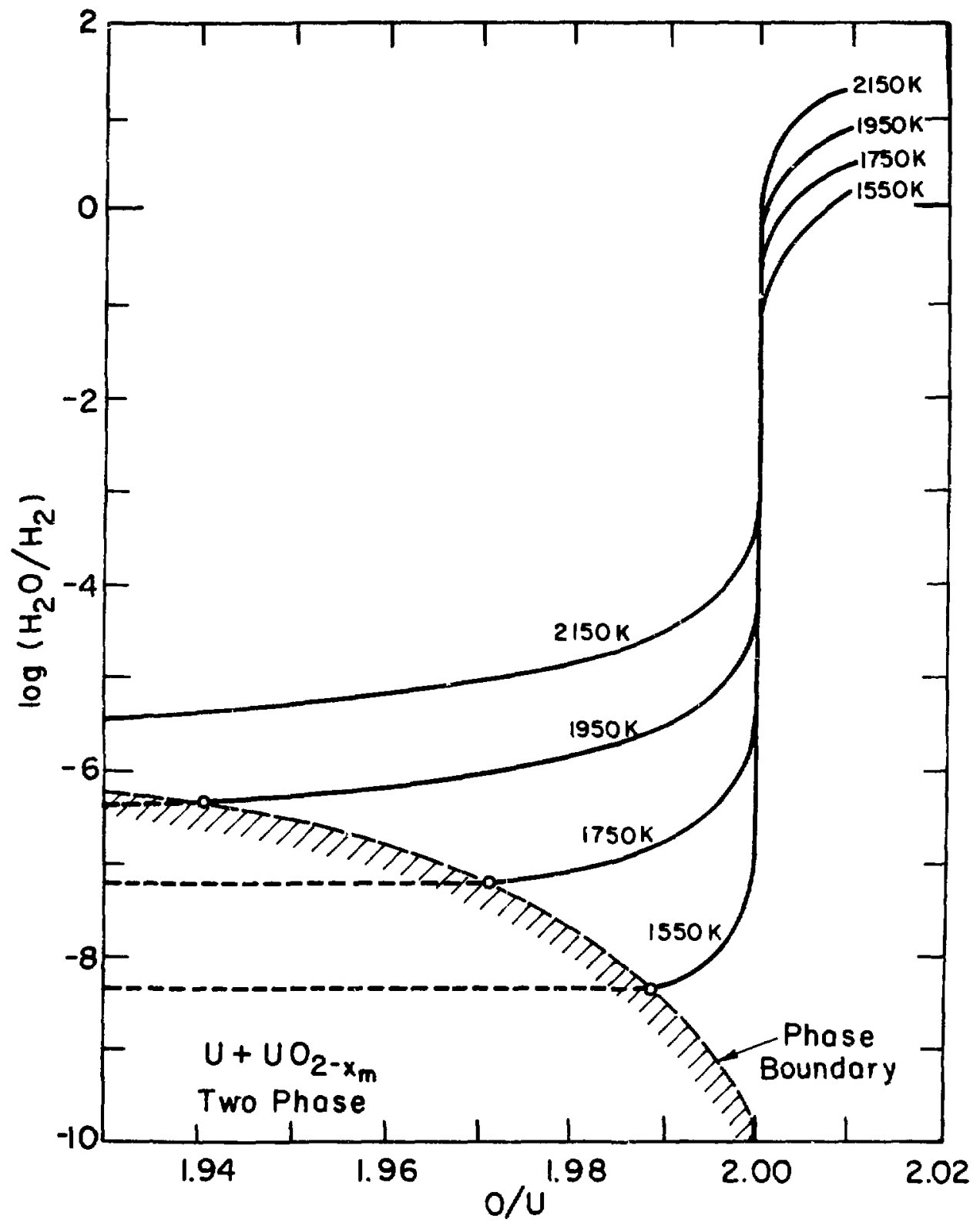

XBL 792-5671

Figure 2. $\mathrm{H}_{2} \mathrm{O} / \mathrm{H}_{2}$ ratios. 


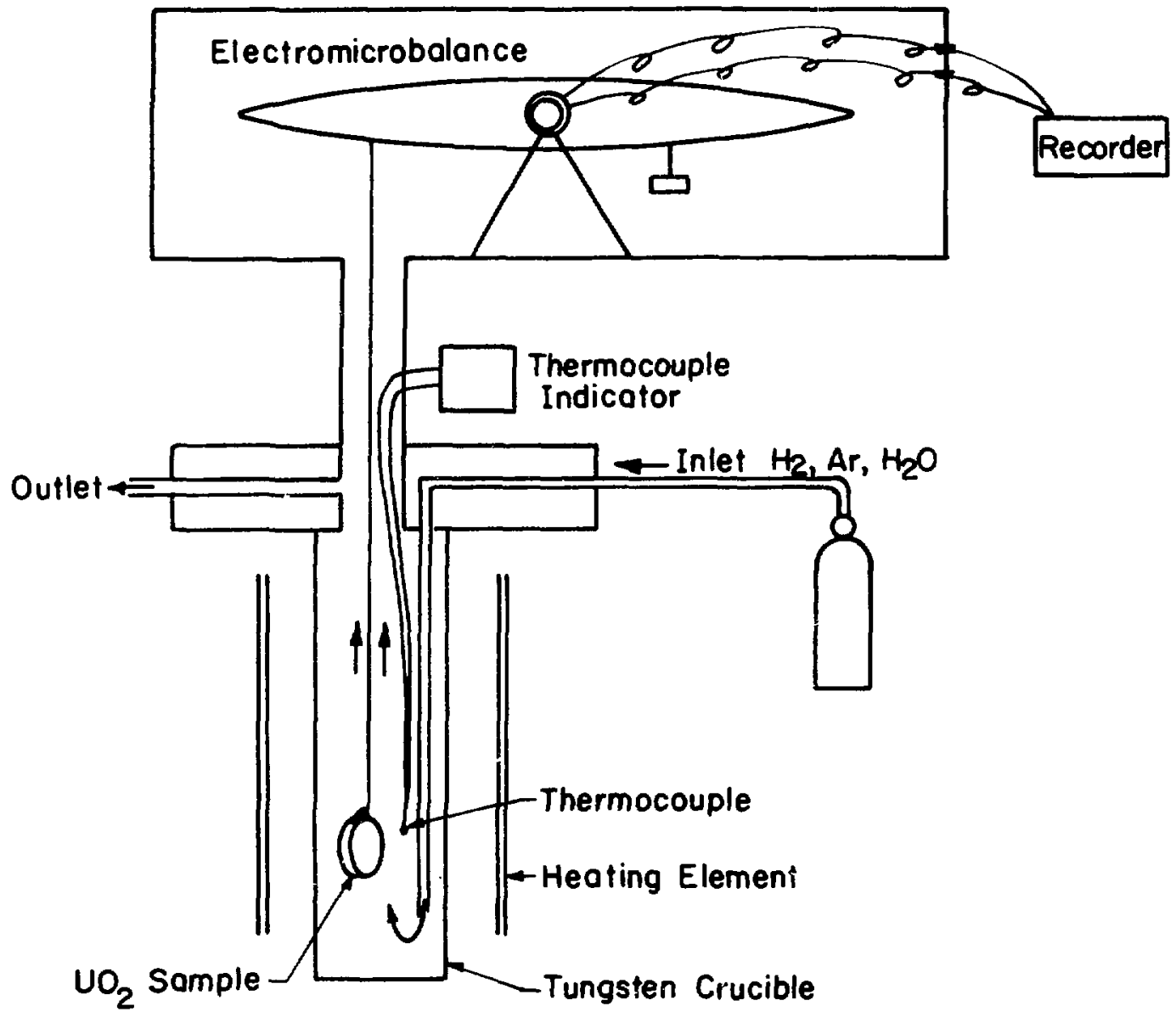

XBL792-5673

Figure 3. Apparatus for reduction/oxidation of $\mathrm{vO}_{2}$. 


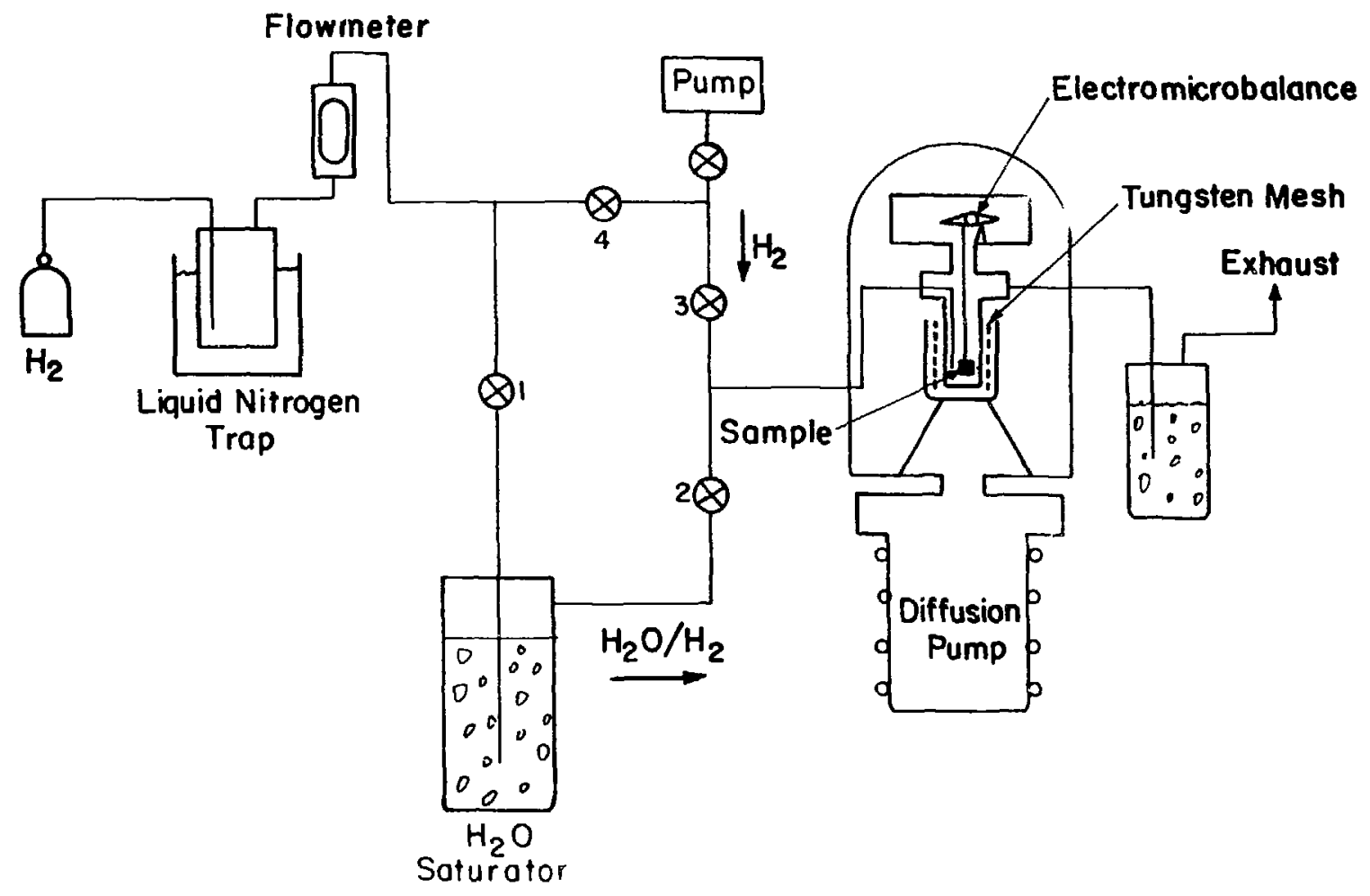

Figure 4. Diagram of gas handling system. 


\section{The Surface Chemistry of Epitaxial Silicon Deposition}

\section{by Thermal Cracking}

\section{By M. Farnaam}

\section{Introduction}

Thermal cracking of silane by the reacticn $\mathrm{SiH}_{4}(\mathrm{~g}) \rightarrow \mathrm{Si}_{\mathrm{i}}+2 \mathrm{H}_{2}(\mathrm{~g})$ is widely used for production of epitaxial layers of silicon on a substrate. This technique is extensively used in the semiconductor industry and in the Fabrication of photovoltaic devices for direct solar energy conversion to electricity. Until a couple of years ago, atmospheric pressure raction was the standard chemical vapor deposition (CVD) growth process. However, the low pressure? chemical vapor deposition (LPCVD) reaction $\left(0.5\right.$ torr $.640^{\circ} \mathrm{C}$ ) was found to give lower costs, higher productivity, and better uniformity compared to the atmospheric pressure method(1). Apparently higher vacuum levels and lower pressures result in a better control of the deposition process, thus encouraging investigators to decrease the pressures and even turn to molecular beam techniques.

Deposition of the epitaxial layers of silicon takes place by the incorporation of silicon adatoms into the growing epitaxial layers, and whether these adatoms are produced from a CVD process or by condensation of silicon vapor or by any other process does not affect the growth process, which is driven by the superstauration of the surface with silicon adatoms. In a CVD process, the adatoms are produced by decomposition of the molecules of the silicon bearing gas on the surface.

Application of the conventional techniques for investigation of the silane-cracking reaction (i.e., measurement of the rate of the steady state production of $\mathrm{H}_{2}$ by heterogeneous reactions) have resulted in contradictory 
mechanistic interpretation for this process $(2,3,4)$. In order to permit more detailed probing of the surface processes than can be achieved by steady state kinetic studies, our experiment utilizes a modulated molecular beam method which has advantages such as phase lag measurements (5).

\section{Experinental}

A. Specimen Preparation

In order to solve the problem of island growth on the crystal surface which was encountered at the hiqh temperatures required for the silane beam-crystal chemical interaction, new techniques and materials were tried. A new type of crystal prepared by the Topsil corporation using methods which are claimed to give much lower impurity concentration than the czochralski method, was utilized. The crystals were undoped in order to avoid possible interference of the dopant in the surface structure during the experiment. The crystals were in the form of disks $5.0 \mathrm{~cm}$ in diameter and $0.25 \mathrm{~cm}$ thick with a (lll) surface orientation. They were cut by diamond saw into slabs $2.8 \mathrm{~cm} \times 0.6 \mathrm{~cm} \times 0.25 \mathrm{~cm}$ in size.

Surface preparation started with polishing with $6 \mathrm{fm}$ diamond paste, cleaning in acetone, methyl ethyl ketone and etranol, dipping in hydrofluoric acid, rinsing in deionized water, blow drying with $\mathrm{N}_{2}$ gas, and finally heating resistively at $1150^{\circ} \mathrm{C}$ in vacuum for about 30 minutes. Scanning electron micrographs of this crystal showed a very rough surface containing irregular islands and pits displaying the trace of scratches made on the crystal during polishing.

Subsequent specimens were polished, cleaned and heated more carefully according to the following treatment:

The crystal was embedded in the top face of a disk of cold mount material about $0.5 \mathrm{~cm}$ thick and polished by hand along at $45^{\circ}$ to the longitudinal direction of the crystal in order to prevent cracking. (0) grade emery paper was used in this step which was continued until the whole surface of 
the crystal was covered only with scratches along the $45^{\circ}$ polishing direction. Then, using a new (0) grade emergy paper, the crystal was polished along the direction perpendicular to the initial direction until the whole surface was covered with scratches in this new direction. After cleaning the crystal ultrasonically, it was polished successively with (00), (000), and (0000) grade enery paper in a similar manner. The polishing was continued using $6 \mathrm{~lm}$ and $1 \mathrm{\mu m}$ diamond paste on the automatic rotary wheel. The last step of polishing was done using vibratory plates covered with microcloth polishing pad and containing $0.05 \mu \mathrm{m} \quad \gamma$-Alumina powder dispensed in distilled water. Ultrasonic cleaning was always used between polishing steps. The crystal was removed from the embedding material by heating with hot air, which causes swelling of the material and releases the crystal without damage. The crystal was cleaned ultrasonically and then degreased in the solution of phosphoric acid ( 3 parts) $\mathrm{H}_{2} \mathrm{O}_{2}(2$ parts). At this stage, the 13-step cleaning method ${ }^{(6)}$ was used for cleaning the crystal.

\section{B. Specimen Heating}

As before, an optical pyrometer was used for measuring the temperatures, hut a heating filament was installed below the ceramic crystal holder. This filament can be heated independently of the crystal and serves two purposes. First, it is used during baking of the system to bake out the whole assembly holding the crystal (ceramic, tantalum clips, wiring, etc...) and the part of the chamber adjacent to the crystal. Secondly, pre-heating of the crystal by the filament causes the resistance of the crystal drop drastically. thereby making it much easier to heat the crystal resistively to high temperatures. 


\section{Results}

A specimen prepared by the method described above was heated in the vacuum system ti $1150^{\circ} \mathrm{C}$ while a molecular beam of silane impinged on the center. Fig. I shows the scanning electron micrographs of this crystal taken after the experiment. It is believed that (7) the triangular vaporization pits on the region of the surface not exposed to the silane beam (Fig. 1-a) results from carbon-contamination. If the surface is clean, vaporization is a 2-D process and no topographic effects occur: in other words thermal etching is the exact inverse of growth. The growth moxphology in the region exposed to silane (Fig. 1-b) is also believed to be an indication of heavy carbon contamination, and at the high temperatures involved this would almost certainly be present as $\beta_{-S} c$.

The crystal used in the experiment was one of the purest types available for epitaxial growth, therefore the bulk of the crystal is not responsible for the contamination. But the crystal surface itself could be the source of the contamination $i . e .$, polishing and cleaning methods should be modified and if necessary new steps must be added.

IV. Planned Experimental Modifications

In-situ $\mathrm{HCl}$ etching is one of the most widely used etching steps for preparing silicon specimens. Therefore a doser for Hcl has been installed in the vacuum system adjacent. to the crystal.

The effect of another step of polishing called Electroless silicon polish will also be investigated. This step is a chemical-mechanical polishing process which will follow polishing with $0.05 \mu \mathrm{m}$-Alumina. 
Another possible solution is the use of epitaxially-grown surfaces, i.e., a film a few microns thick grown on a substrate by conventional silicon process technology. This material will simply be mounted in the vacuum system and heat cleaned for a few minutes at $1100-1200^{\circ} \mathrm{K}$. Which is claimed to be adequate to remove the carbon (from adsorbed atmospheric gases) to below the detection limit of several usual detection techniques $(7,8)$. The vacuum system may also be modified (e.g. by replacing the diffusion pump by a cryopump) to reduce contamination due to background gases.

Once pitting evaporation is eliminated and 2-D cvaporation and growth is achieved, the kinetic studies of the silane cracking reaction will be continued.

\section{References:}

1. R.S. Rosler, Solid State Tech. P 63 April (1977).

2. B.A. Joyce et. al. Phil. Mag 14, 289, 301 (1966) and 1.167 (1967).

3. R.C. Henderson et. al. Surf. Sci. 30, 310 (1972).

4. R.F.C. Farrow, J. Electrochem. Soc. 121899 (1974).

5. R.H. Jones, D.R. Olander, W.J. Siekhaus, and J.A. Schwarz J. Vac. Sci. Tech. 91429 (1972).

6. S.P. Murarka et. al. J. Appl. Phys. 48 No. 9, (1977).

7. Private Communications with B.A. Joyce.

8. R.C. Henderson et. al. J. of Appl. Phys. 42, No. 3 (1971). 


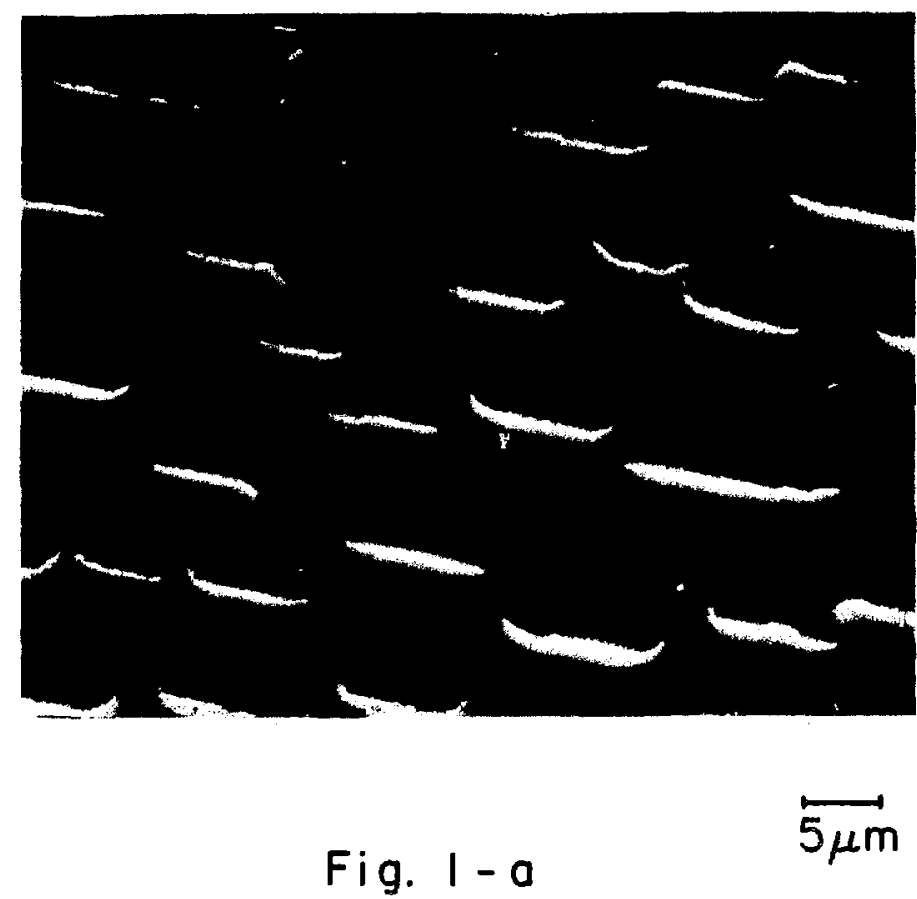

Crystal \#17 Area not exposed to silane

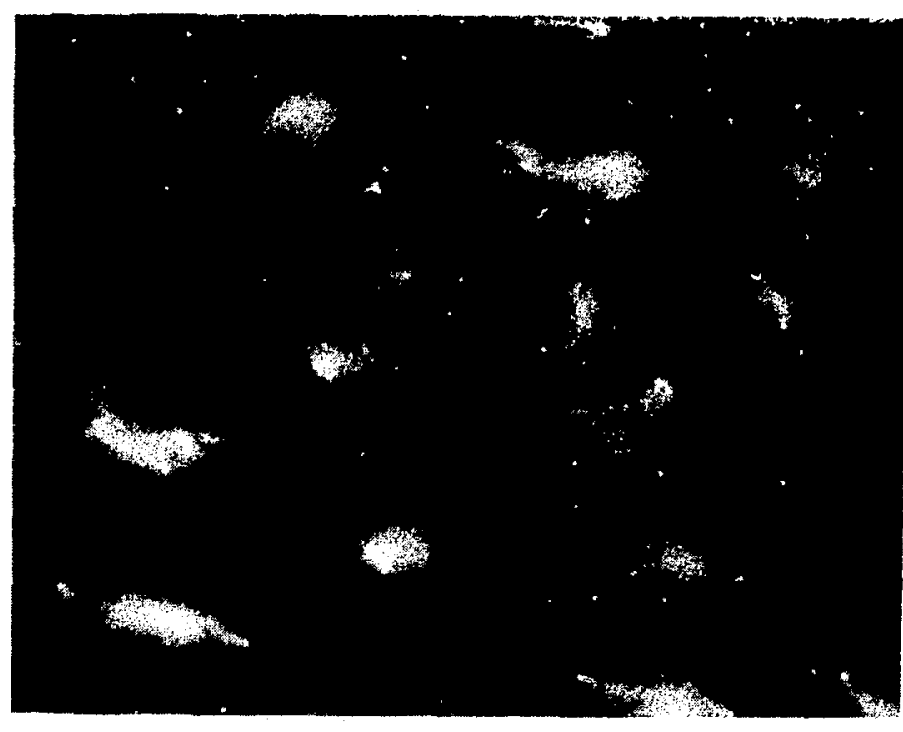

Crystal \#17 Area exposed to silane 
The Kinetics of Laser Pulse Vaporization of $\mathrm{NO}_{2}$

by C.H. Tsai

\section{Introduction}

The laser pl se vaporization technique has been developed recently to investigate the altra-high temperature thermodynamic properties of $\mathrm{uO}_{2}$. $(1-3)$ The experimental $\vec{r}$ termination of this thermodynamic information is necessary because of the great inconsistency in theoretical calculations as mentioned in the previous rer ort.

Two techniques, namely the photographic and mass spectrometry techniques, have been conducter independently for vapor pressure interpretation. The transient optical $\mathrm{p}$ ? rometry is used for surface temperature measurement in each of the methods A computer code has been developed to calculate the surface temperature and surface composicion of uranium oxide during the laser evaporation transient in order to compare with the experimental measurements.

The current y ar was devoted to the vapor phase angular dis .ribution determination and surface temperature measurement by optical pyrometry, in which the mass s ectrometry technique is ultilized. Some preliminary results of these measuremen $s$ are given.

\section{II . Experiments}

A sketch of t.e experimental set up of laser pulsing on $\mathrm{UO}_{2}$ is shown in Fig. la. 
A UO 2 sample is preheated by electron-beam bombardment heater to ר. $1400^{\circ} \mathrm{C}$, the ductile-to-brittle transition temperature of $\mathrm{UO}_{2}^{(4)}$, in order to avoid sample cracking resulting from the huge thermal gradient induced by laser heating. At this temperature, the absorption cut-off of $\mathrm{NO}_{2}$ is also shifted to $1.3 \mu \mathrm{m}$ wavelength (longer than the wavelength of Nd-glass laser 3.06 $\mathrm{\mu m}$ ) which avoids in-depth heating by laser radiation penetration into the target.

The laser beam is partially split to a Mgo diffuser and absorbed by a pre-calibrated photodiode which gives the power shape as well as the power as a function of time. The laser power together with the laser beam radial intensity profile measured by knife-edge technique as mentioned in the last year report gives the laser peak maximum power density of each experimental run, which provides the input data for corresponding computer run for the temperature calculation.

A single-wavelength $(0.65 \mu \mathrm{m})$ transient optica? r.yrometer is used to measure both the surface temperature of the pre-haated target and the surface temperature during laser heaiing. The transient optical pyrometer calibration is performed by a master manual optical pyrometer pre-calitrated by NBS and a graphite black-body cavity with the capacity of reaching $3000^{\circ} \mathrm{C}$. Above this range, the absorbing glass filters are used to establish the extrapolation uf, to $6000 \mathrm{~K}$.

Two arrays of small teflon disks and aluminum disks are placed around the target by a collector assembly mounted in $7-$ dirertinn to collert part nf the ejected $\mathrm{UO}_{2}$ from laser-heated spot, as shown in Fig. la \& lb. A stainless steel flag is controlled by a linear feedthrough in $x$-direction to avoid vapor deposits on the collector disks during taryet pre-heating and it is removed be "ore laser shots. The teflon disks are then irradiated in the Berkeley Research Reactor to determine the angular distribution of vaporized $\mathrm{UO}_{2}$ by counting the fission product radioactivity and to provide an independen: 
estimate of the total amount of $\mathrm{UO}_{2}$ vaporized. Calibrated standards consisting of known amount of uranium from uranyl nitrate solution deposited on teflon disks are used to determine the absolute magnitude of the quantity of uri.jium deposited on the disks by the laser evaporation process. The aluminum disks are examined microscopically to determine whether the ejected material consists entirely of vapor species or emission of liquid droplets occurs.

\section{Results}

A. Laser Light Absorption by the Vapor Plume

The degree of thermal ic ization in the vapor cloud above the surface has been estimated to be $2.7 \%$ at $4400 \mathrm{~K} .^{(8)}$ The partially ionized vapor plume does not satisfy the double inequality characterizing an ordinary kinetic rlasma ${ }^{(8)}$, so the optical absorption is dominated by Bremsstrahlung absorption. According to the Bremsstrahlung absorption theory ${ }^{(9)}$ assuming all the ions are singly charged, the absorption coefficient is $0.0466 \mathrm{~cm}^{-1}$ for $1.06 \mu \mathrm{m} \mathrm{Nd-glass}$ laser and $0.0107 \mathrm{~cm}^{-1}$ for $0.65 \mu \mathrm{m}$ optical pyrometer. Assuming an absorbing layer of $0.5 \mathrm{~mm}^{(10)}$, the absorption of $1.06 \mu \mathrm{m}$ laser 1 ight is 0.28 and that of $0.65 \mu \mathrm{m}$ optical pyrometer is 0.158 . It is obvious that the optical absorption is negligible in thi:; temperature range.

\section{B. Temperature Measurement}

The maximum surface temperature measured by the transient optical pyrometer is $4200 \pm 60 \mathrm{~K}$ for the run at laser total energy of 27.85 Joules. After taking the pectral emissivity of $\mathrm{UO}_{2}$ at this temperature at $0.65 \mu \mathrm{m}$ ( $0.92^{(11)}$ ) into account, the maximum surface temperature is about $4268 \mathrm{~K}$. The result of the temperature calculation by our computer code is $4410 \mathrm{~K}$ and $4200 \mathrm{~K}$ with and without considering surface composition depletion respectively. The focal distance of the optical pvrometer is $\sim 53 \mathrm{~cm}$, and the diameter of the focal spot corresponding to this focal distance is ح $1.33 \mathrm{~mm}$. Since the optical pyrometer is sighted $45^{\circ}$ to the target normal, the pyrometer focal spot is elliptical with a major axis of $\sim 1.88 \mathrm{~mm}$. 
According to the laser power intensity profile across radial direction on the tarqet measured by knife-edqe technique, the error of the optical purometer measurement corresponding to this power variation across the pyrometer focal spot is only $5 \mathrm{~K}$ (see Fig. 2).

In order to verify the temperature measurement, graphite and tungsten are being studied to compare the experimental measurement and theoretical calculation by taking the advantages of well-known high temperature properties of both materials.

\section{Target Characterization}

The irradiated $\mathrm{UO}_{2}$ target has been examined by scanning electron microscope. Fig. 3a shows the laser-irradiated area. Melting out to ar: ellipse of major radius $3.81 \mathrm{~mm}$ and minor radius $2.35 \mathrm{~mm}$ is seen in this micrograph. An apparent ring structure on the target surface is observed within the molten area. The ring structure is attributed to the radial propagation of a central disturbance on the liquid surface in the form of capillary waves. (12) The discontinuous quasi-periodic liquid displacement from the centre of the "pool" due to the recoil force of the evaporating material is believed to be the driving mechanism of this disturbance.

Figs. $3 \mathrm{~b}$ and $3 \mathrm{c}$ show the $\mathrm{UO}_{2}$ surface under higher magnification with and without me ting respectively. The white dots appearing mostly along the grain boundaries in the irradiated area (Fig. 3b) probably uranium metal aggregates which are expected due to the preferential evaporation of oxygen under s rong free evaporation.

D. Deposits on the Aluminum Collectors

Figs. $4 a$ and $4 b$ show scanning electron micrographs of the aluminum disk collectors. It is evident that there is condensed $\mathrm{UO}_{2}$ on the disks, some with donut shape and some spherical. The radii range from 1 fum to $15 \mu \mathrm{m}$. Elemental analysis (EDAX) result (Fig. 4C) reveals that they contain uranium, which suggests that either: (a) $\mathrm{UO}_{2}$ liquid-droplet ejection 
from the melt occurs -- this is also suggested by the small craters in the central area in Fig. 3a =- or (b) liquid droplets are formed by condensation in the collision-dominant continuum layer of the vapor plume.

E. Angular Distribution

A single $\mathrm{UO}_{2}$ target was subjected to five laser shots to measure the vapor angular distribtion by teflon disk collectors. The amount of $110_{2}$ collected on each teflon disk ranges from $15 \mu \mathrm{g}$ to $60 \mathrm{\mu q}$, whach curre'sponds to about $120 \AA$ to $480 \&$ thickness of $\mathrm{UO}_{2}$ layer if uniformly deposited. Taking into account the geometry of the collectors, the total amount of $10_{2}$ cvaporated is estimated approximately $2.2 \mathrm{mg}$, compared to the theoretical calculation of about $7.9 \mathrm{mg}$ assuming Langmuir evaporation.

The angular distribution of mess flux from $v_{2}$ vaporization is shown in Fig. 5. This plot shows that the angular distribution corresponds quite well with the $\cos ^{2} 0$ distribution. It has been found (6) that the angular variation of the supersonic free jets from sonic: orifices obey a $\cos ^{2} 1$ relationship. This measurement reveals that the flow is not an equilibrium Maxwellian distribution; the vapor flow from strong free evaporation into vaccum cas be simulated by a supersonic free-jet from a sonic orifice which is consistent with the conclusion of dynamic sampling method iy mass spectrometry. (7) 


\section{References}

1. R.W. Ohse, P.G. Barrie, H.G. Bogensberger and E.A. Fischer, IAEA Symp. on Thermodynamics of Nuclear Materials, Paper IAEA-SM-190/8, Vienna Oct. 1974 .

2. A. Bober, J.U. Karow ana K. Schretzmann, IAEA Symp. on Thermodynamics of Nuclear Materials, Paper IAEA-SM-190/34, Vienna Oct. 1974.

3. N. Asami, M. Nichikawa and M. Taguchi, IAEA Symp. on Thermodynamics of Nuclear Materials, Paper IAEA-SM-190/3, Vienna Oct. 1974.

4. D.R. Olander, "Fundamental Aspects of Nuclear FEactor Fuel Elements", ERDA Technical Information Center, Oak Ridge, Tennessee April 1976.

5. M.J. Davies, Ph.D. Thesis, Dept. of Inorganic and Structural Chemistry. University of Leeds, Jan. 2970.

6. H. Ashkanas and F.S. Sherman, Rarefied Gas Dynamics - 4 th Symposium, Vol. II, PP, 84-105, 1966.

7. K.A. Lincoln and M.A. Covington, Int. J. of Mass Spectrometry and Ion Physics, 16 (1975) 191-208.

8. H.U. Ka row, KFK-2390, Feb. 1977.

9. Ya.B. Zel'dovich and Yu.P. Raizer, "Physics of Schock Waves and HighTemperature Hydrodynamic Phenomena", Vol. I, Academic Press, New York and London 1966.

10. R.W. Ohse and P.G. Berrie, J. Nucl. Mater. 59 (1976) 112-124.

11. M. Bober and H.U. Karow, 7th Symposium on Thermophysical properties, NBS Washington, D.C., 1977.

12. J. Magill and R.W. Ohse, J. of Nucl. Mat. 71 (1977) 191-193. 


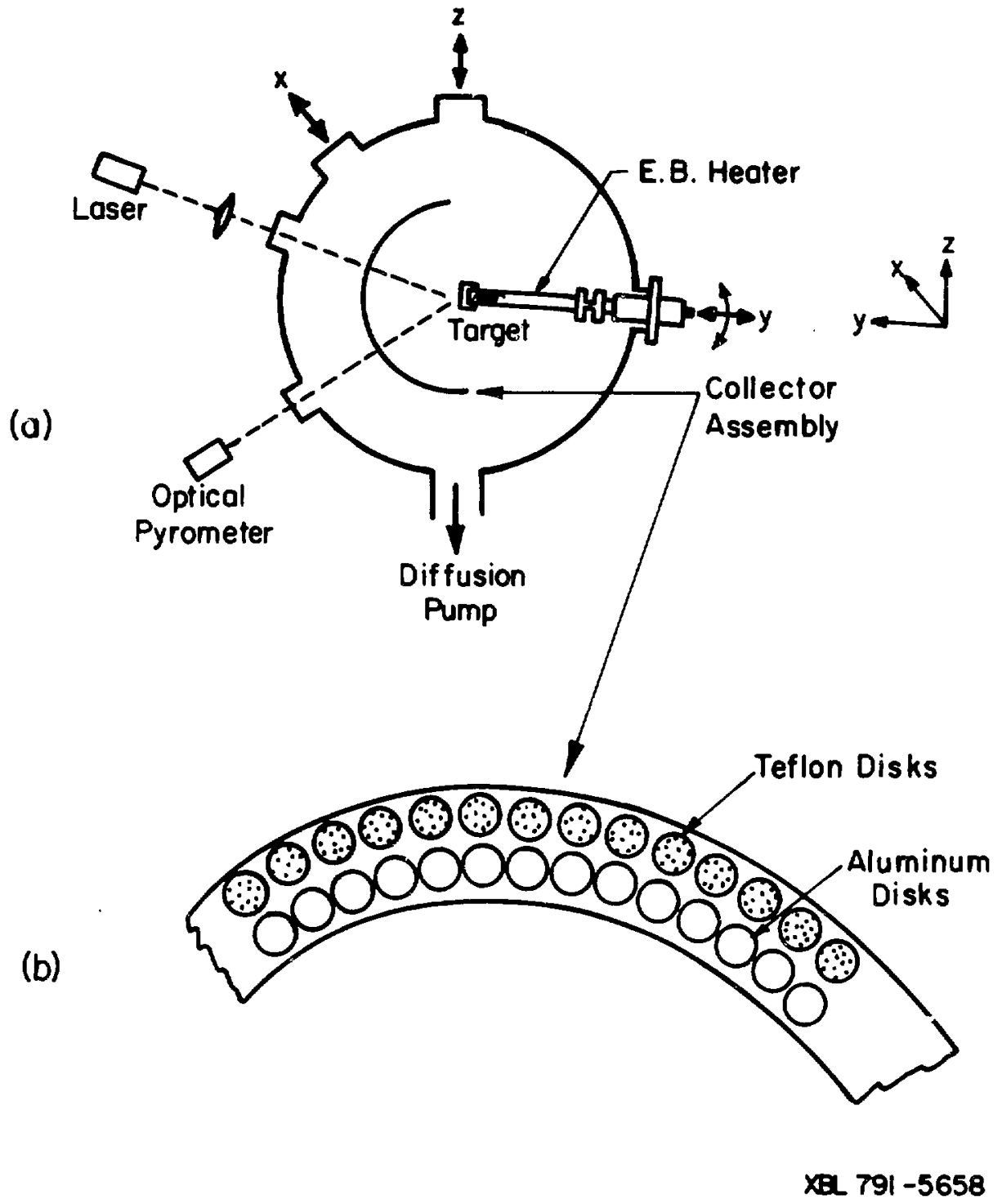

Figure 1. The collector assembly for angular distribution measurement and liquid droplet collection in LBL's apparatus. 


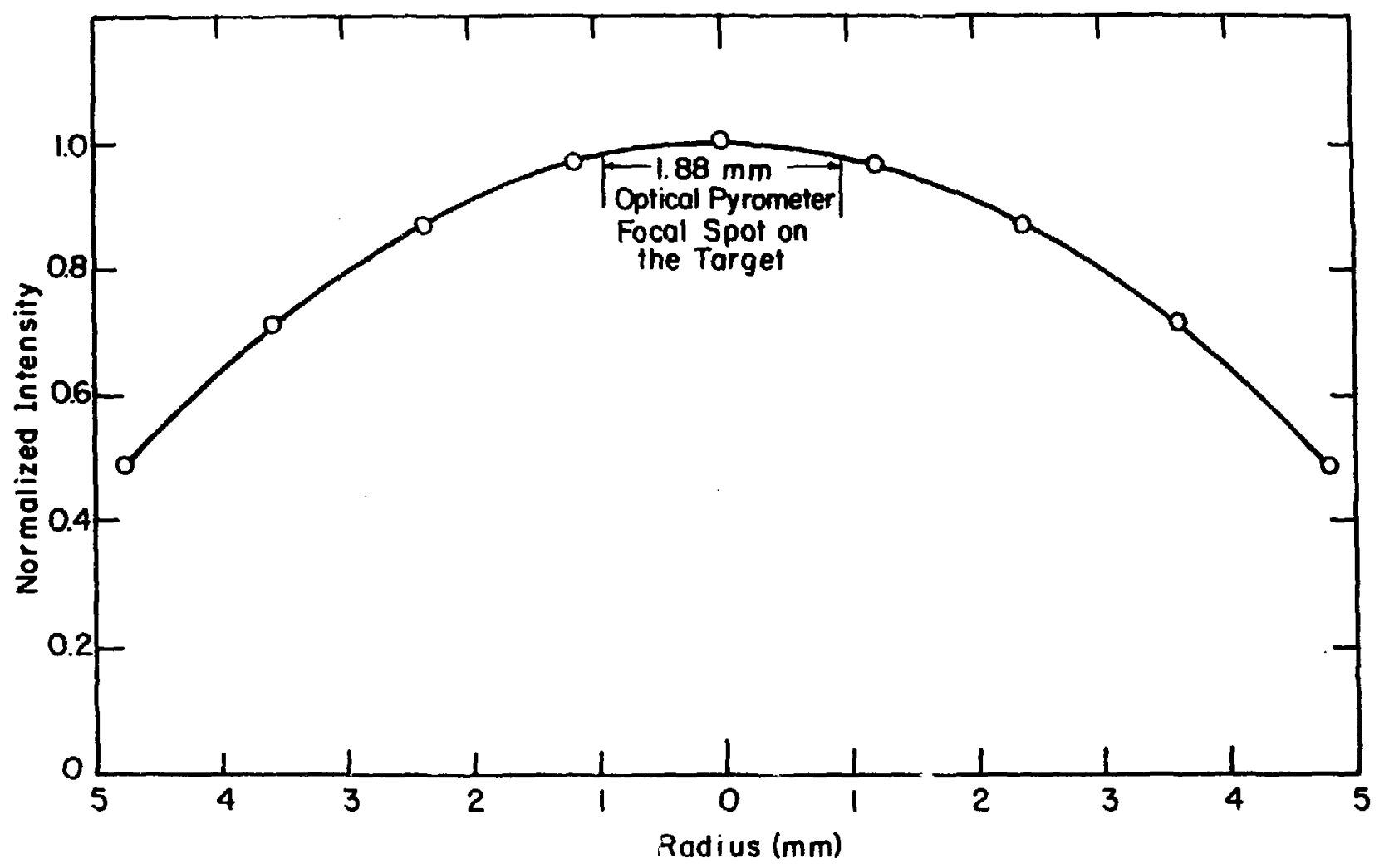

XBL $791-5659$

Figure 2. Normalized laser power intensity radial profile. 

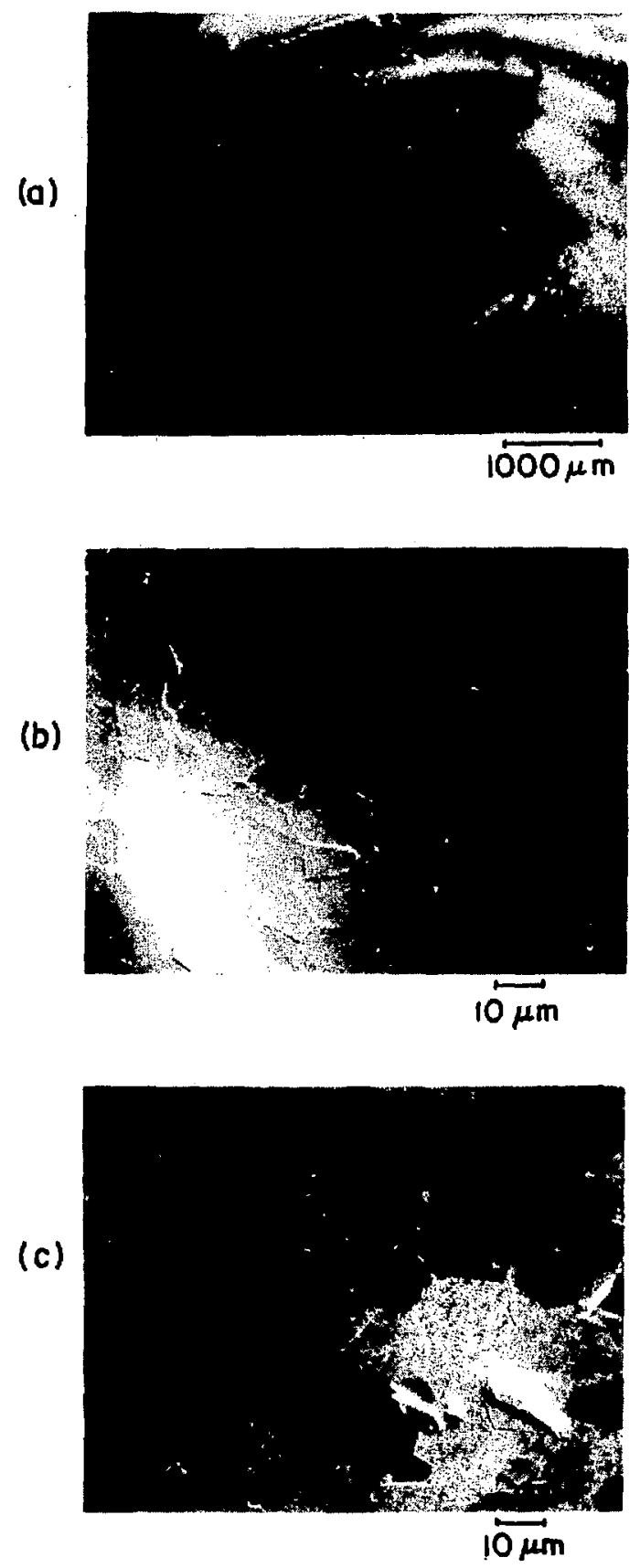

XBB 792-1258A

Figure 3. Scanning electron microphotographs of molten $\mathrm{UO}_{2}$ surface (a) and $(b)$, and non-molten surface (c). 


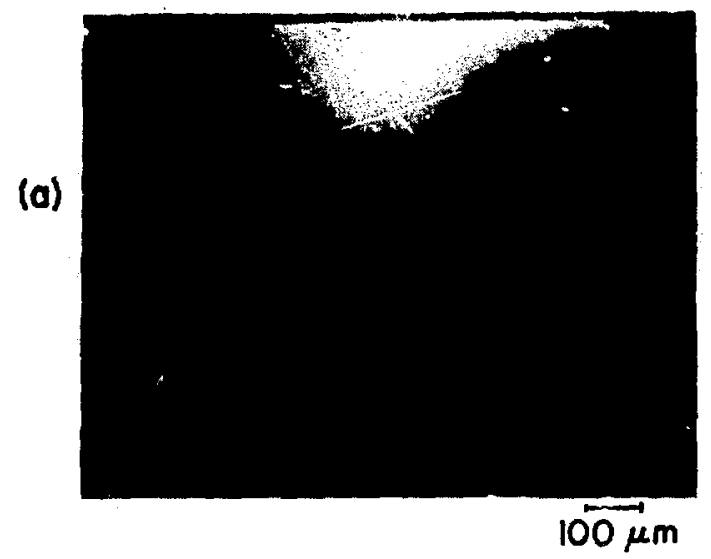

(b)

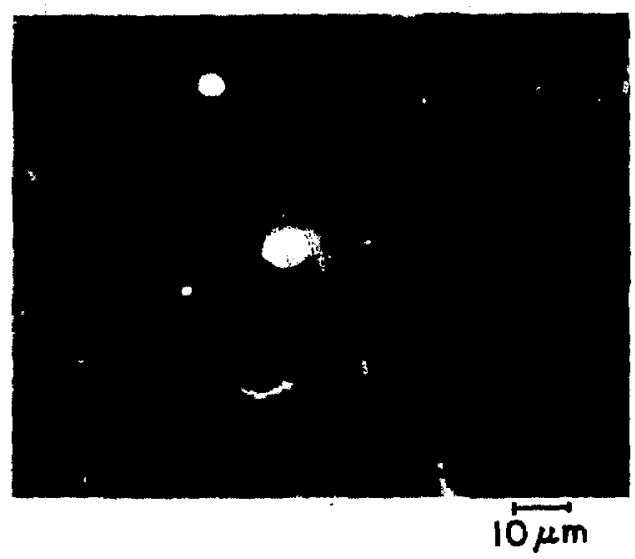

(c)

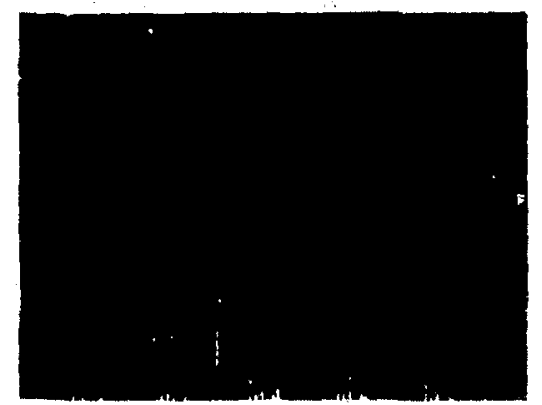

XBB 792-1257A

Figure 4. Scanning electron microphotographs of aluminum disk collector surface (a) and (b), and the EDAX analysis (c). 


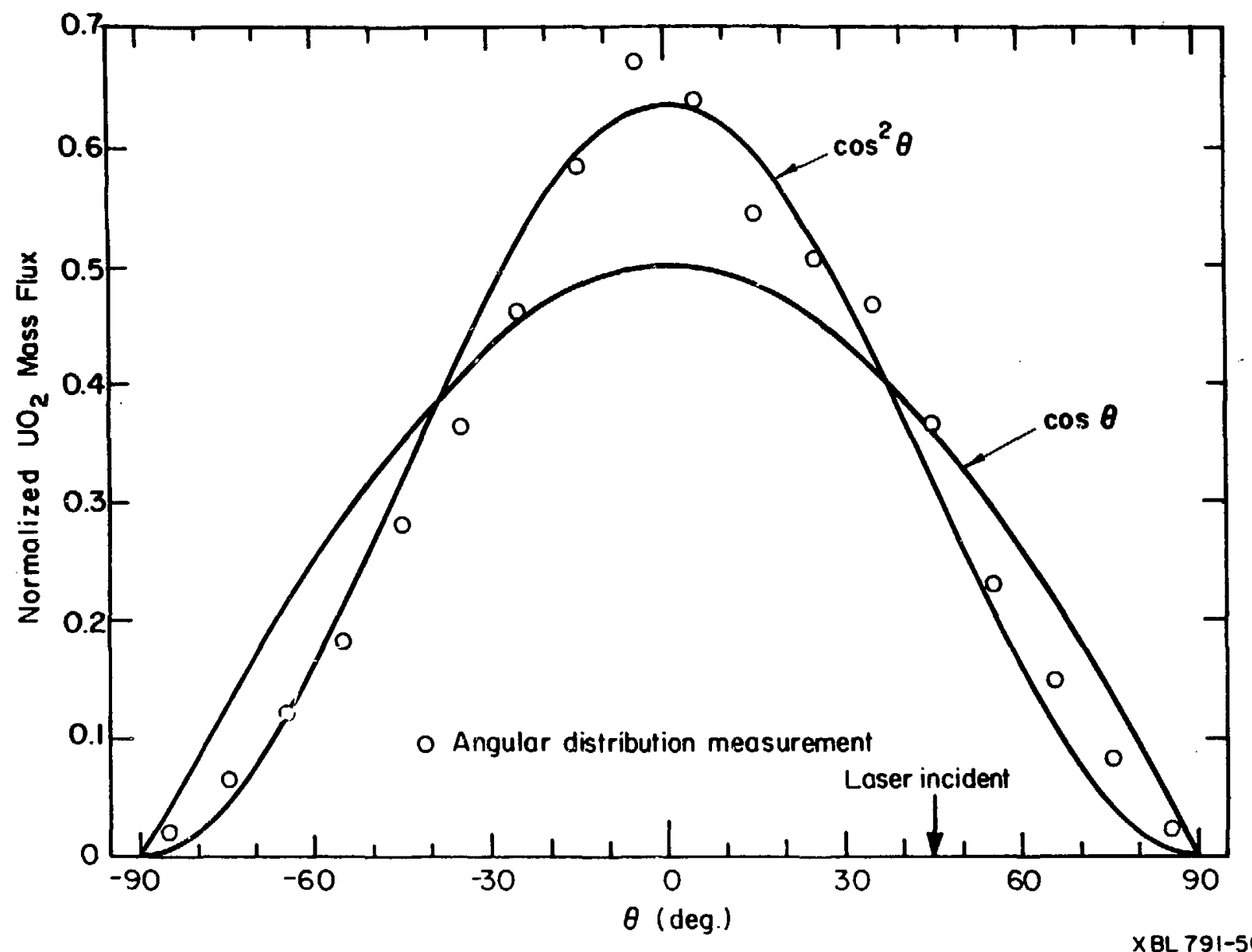

Figure 5. Angular distribution of mass flux in vapor plume from $\mathrm{UO}_{2}$ vaporization. 


\section{RETENTION AND RELEASE OF WATER VAPOR BY URANIUM DIOXIDE}

$$
\text { by Douglas sherman }
$$

\section{INTRODUCTION}

Uranium dioxide is known to absorb water vapor from the air. When the $\omega_{2}$ is subsequently heated the water is released. This water is a potential source of hydrogen which can cause hydriding of zircaloy cladding. In earlier experiments, (1-3) degassing of $\mathrm{UO}_{2}$ which had been stored in air resulted in predominantly low-temperature release of water vapcr.

In recent work in this laboratory (4) on the retention and release kinetics of water vapor by $\mathrm{UO}_{2}$, the samples were infused with $\mathrm{D}_{2} \mathrm{O}$ instead of $\mathrm{H}_{2} \mathrm{O}$ in order to avoid the large $\mathrm{H}_{2} \mathrm{O}$ background in the vacuum system. In addition, the release of $\mathrm{D}_{2} \mathrm{O}$ during degassing was monitored in situ by a mass spectrometer instead of a post-release transfer of the gas to a separate chamber for analyses, as in Refs. 1-3.

The experimental technique has been described in the previous repurt. (4) Briefly it consists of monitoring the release of a modulated molecular beam of $\mathrm{D}_{2} \mathrm{O}$ by a mass spectrometer (Fig. 1). The beam is produced by heating the pellet.s in a refractory metal crucible which is suspended in a tungaten mesh furnace. The entire crucible assembly is kept at eleveated temperatures of up to $600^{\circ} \mathrm{C}$ so that no $D_{2} O$ can be absorbed on the inner walls. The nutgassed $D_{2} O$ then effuses from the exit capillary and is chopped, thus producing a modulated molecular beam. The output from the mass spectrometer which detects this molecular beam is fed into a lock-in amplifier which eliminates the d.c. component of the signal, thereby achieving a sensitivity of $-10^{-1 ?} \mathrm{~g} \mathrm{D}_{2} \mathrm{O} / \mathrm{sec}$. The mass spectrometer is calibrated with a known flow rate of neon.

The experiment reported in Ref. 4 was conducted over the range of room 
temperature to $1600^{\circ} \mathrm{C}$, using a platinum crucible. A total of $12 \mu \mathrm{g} \mathrm{D}_{2} \mathrm{O} / \mathrm{g} \mathrm{UO} 2$ was reported to have been released, $95 \%$ of it at temperatures above $1000^{\circ} \mathrm{C}$. This year, an attempt was made to reproduce these results. In this duplicate experiment, similar results to those reported earlier were observed. In addition, however, it was decided to investigate to see if the mass spectrometer signal really corresponded to $\mathrm{D}_{2} \mathrm{O}$ release. The mass spectrometer was tuned to mass 9 where no signal could be expected. On raising the voltage in the furnace, a burst in the signal was observed of similar magnitude as that of mass 20, which was supposed to be $D_{2} \mathrm{O}$. Since no signal should have been detected at mass 9 , this indicated that the high temperature "release of $\mathrm{D}_{2} \mathrm{O}$ " that had been observed here and in the first experiment was due entirely to electronic pickup from the furnace.

To avoid electronic pickup by the mass spectrometer, an aluminum sheath which encased the mass spectrometer (except for a hole to admit the molecular beam) was installed. A blank was run and no appreciable signal was detected, even at high temperatures. A neon calibration was run to insure that the mass Epectrometer could dotoct mass 20 singals.

\section{EXPERIMENTS OF JULY 1978}

Three different types of $\mathrm{UO}_{2}$ pellets of known open and closed porosity were prepared by Exxon Nuclear Co. These are designated as runs 1, 2, and 3 in Table 1.

The samples were outgassed at $250^{\circ} \mathrm{C}$ for 24 hours in vacuum, cooled and immersed immediately in liquid $\mathrm{D}_{2} \mathrm{O}$ for $2-4$ hours. Excess $\mathrm{D}_{2} \mathrm{O}$ was removed by rolling in a lint-free towel, and the samples were transferred to the crucible and placed in the vacuum system. To remove any loosely bound surface $\mathrm{D}_{2} \mathrm{O}$ the samples were evacuated at room temperature for 18 hours prior to the temp- 
erature increase.

To measure the background, a sank (empty crucible) was run for the entire temperature range. During the outgassing the temperature was ramped up continuously instead of in disarete steps. In Run 1, the high open and closed porosity sample was outgassed for 5.5 hours with a linear temperature ramp of $300^{\circ} \mathrm{C} / \mathrm{hr}$. As seen in Fig. 2 tre release rate had a major peak at $120^{\circ} \mathrm{C}$ of $480 \mu \mathrm{g} / \mathrm{hr}$. This decayed to $15 \mu \mathrm{g} / \mathrm{hr}$ by $30 \mathrm{C}^{\circ} \mathrm{C}$. The background rate of approximately $0.3 \mu \mathrm{g} / \mathrm{hr}$ was reached at $1100 \mathrm{C}$ and persisted at higher temperatures. A total of $17 \mathrm{Hg} \mathrm{D}_{2} \mathrm{O}$ $9 \mathrm{UO}_{2}$ was released with 948 appearing at temperatures $<300^{\circ} \mathrm{C}$. The large amount of absorbed $\mathrm{D}_{2} \mathrm{O}$ is due to the high open porosity of this sample.

Samples 2 and 3 were outgassed 6.5 and 1.8 hours at linear temperature increase rates of $250^{\circ} \mathrm{C} / \mathrm{hr}$ and $900^{\circ} \mathrm{C} / \mathrm{hr}$ respectively. For these two samples, which had low open porosity, the amount of $\mathrm{D}_{2} \mathrm{O}$ released was negligible (see Fig. 3 ). In Run 2 the release rate was at or below background. In Run 3 the release rate was double the background level determined priox to Run 2, but because of uncertainties in calibration of the two runs, this release rate is probably at background as well. For both samples there was no observed release of $\mathrm{D}_{2} \mathrm{O}$ at high temperature. From these experiments, it appears that water adsorbed on sintered pellets is only loosely bound. A porous sample (no. 1), absorbed a great deal of water, but the moisture was removed at very low temperature. In the samples with low open porosity (nos. 2 and 3) no $\mathrm{D}_{2} \mathrm{O}$ was observed at any temperature, sviously because very little $\mathrm{D}_{2} \mathrm{O}$ had been absorbed. No effect of closed porosity was observed.

\section{POROSITY MEASUREMENTS}

The open and closed porosity of some of the $\mathrm{UO}_{2}$ samples was determined using a standard technique. (5) The samples were infused with $\mathrm{H}_{2} \mathrm{O}$ in the apparatus shown in Fig. 4. The sampies were first heated in vacuum at $250^{\circ} \mathrm{C}$ for 24 hours. 
They were then cooled and weighed. The samples were replacnd in the apparatus and huated in vacuum for 24 hours to $250^{\circ} \mathrm{C}$, then cooled and immediately immersed in $\mathrm{H}_{2} \mathrm{O}$. The samples were rolled ir. a lint-free towel to remove excess water and then weighed. The difference in the two weights divided by the dry weight is the open porosity. The closed porosity is determined by the suspension technique where samples are weighed while suspended in watex. The difference between the suspended and ary weights divided into the dry weight gives the closed porosity. Four different samples were measured. All had been used in the previous investigation (4). The results are compared to these obtained at BNWL (Table 2), who used an immersion technique on the same samples to determine their porosity (6).

IV EXPERIMENTS OF DECEMBER, 1978

In order to observe high temperature release of $\mathrm{D}_{2} \mathrm{O}$ it is believed that the $\mathrm{UO}_{2}$ had to be infused with $\mathrm{D}_{2}$ and/or $\mathrm{D}_{2} \mathrm{O}$ at high temperature. Exxon Nuclear Co. prepared $\mathrm{NO}_{2}$ pellets by sintering in a $\mathrm{D}_{2}-\mathrm{D}_{2} \mathrm{O}$ atmosphere at $1720^{\circ} \mathrm{C}$ (used in Runs 4, 5, and 7, see Table 1).

These pellets were heated up to $2740^{\circ} \mathrm{C}$, very close to the melting point, to investigate the high teluperature release of $\mathrm{D}_{2} \mathrm{O}$ and $\mathrm{D}_{2}$. The whole assembly above the heater approached temperatures of $600^{\circ} \mathrm{C}$ at the highest furnace temperatures. The high temperatures of the furnace was monitored with an optical pyrometer.

lour different runs were conducted (see Table 1). The first two, denoted as runs 4 and 5 , were conducted using pellets sintered in $D_{2} O / D_{2}$ and the release of $\mathrm{D}_{2} \mathrm{O}$ was monitored. Run 6 was a blank which used a pellet from the same batch as run 2 which had been sintered in $\mathrm{H}_{2} \mathrm{O} / \mathrm{H}_{2}$ and had shown no appreciable release of $\mathrm{D}_{2} \mathrm{O}$ in a previous outgassing. The last run, number 7 was conducted using the same pellets as used in runs 4 and 5 , but the mass spectrometer was tuned to mass 4 to detect release of $\mathrm{D}_{2}$. 
Run 4 was conducted for 9.3 hours. The results are shown in Figs. 5 and 6. Because low temperature water release was not of great interest, the temperature of the crucible was raised rapidly to $910^{\circ} \mathrm{C}$. The signal reached a maximum of $107 \mu \mathrm{g} / \mathrm{hr}$ within 1.8 minutes. The system was kept at this temperature until the signal decreased to $3 \mu \mathrm{g} / \mathrm{hr}$. The rest of the outgassing was conducted with the temperature being raised in discrete steps, the signal reaching a maximum and then decaying to almost background. The signals observed during the $2440^{\circ} \mathrm{C}$ and $2640^{\circ} \mathrm{C}$ anneals were different in character from the lower temperature releases in that the signal did not immediately reach a maximum and then decay exponentially. This may be due to the sample changing phase with the opening of the closed pores or some other internal reorganization of the sample. On raising the temperature from 2700 to $2720^{\circ} \mathrm{C}$ no more $\mathrm{D}_{2} \mathrm{O}$ was observed and the experiment was terminated. A total of $46 \mu \mathrm{g} \mathrm{D}_{2} \mathrm{O}$ was released in the outgas with $\sim 24 \mathrm{~kg} \mathrm{D}_{2} \mathrm{O}$ being released at temperatures $<1000^{\circ} \mathrm{C}$.

Run 5 was an attempt to duplicate run 4 with similar temperature increments. To see more temperature behavior the first outgas was at $550^{\circ} \mathrm{C}$, with subsequent anneals of 910 and $1125^{\circ} \mathrm{C}$. The other anneals were similar to those in run 4 . The results of run 5 are shown in Fig. 7 and 8 . The maximum signal was observed to be $38 \mathrm{~kg} / \mathrm{hr}$. Like the previous run, the very high temperature outgasses showed a different behavior with no imnediate rise followed by decay. In addition, there was a sharp rise during the $2450^{\circ} \mathrm{C}$ outgas after an initial rise had been observed. This burst in signal was probably due to some change of phase or structure in the sample which car jed a sudden $\mathrm{D}_{2}$ O release from the sample. After seeing no appreciable rise in signal at $2760^{\circ} \mathrm{C}$ the experiment was terminated after 6.5 hours of outgassing. The total $\mathrm{D}_{2} \mathrm{O}$ observed was $17 \mathrm{\mu g}$ with $7.5 \mathrm{\mu g}$ of that above $1000^{\circ} \mathrm{C}$. This is less than in run 4 with the difference due to some experimental uncertainties and possible differences in the samples. 
Run number 6, which is the background run, is superimposed on Figs. 5 and 7 in dashed lines. It is seen that at the end of the outgas the signal had almost reached background.

In order to observe the release of $D_{2}$ from the samples in run 7 the mass spectrometer was tuned to mass 4. The mass spectrometer was calibrated with a known flow rate of deuterium prior to the outgassing experiment. The outyas was conducted similarly to that of runs 4 and 5 but for a much smaller time. Due to the much higher diffusivity of $\mathrm{D}_{2}$ as opposed to $\mathrm{D}_{2} \mathrm{O}$, the anneals at individual temperatures were short because the $D_{2}$ was quickly released after the temperature had been raised. In addition, no $\mathrm{D}_{2}$ was observed at low temperatures, so the temperature was quickly raised to $1620^{\circ} \mathrm{C}$, then increased in discrete steps as before. The experiment was terminated after an outgassing of 161 minutes. As before, a sudden pulse of $\mathrm{D}_{2}$ was observed during the middle of the $2590^{\circ} \mathrm{C}$ anneal. This burst occurred at about the same temperature as the $\mathrm{D}_{2} \mathrm{O}$ burst in run 5 and may again be due to the sudden opening of closed porosity in the $\mathrm{UO}_{2} \mathrm{sample}$ Accounting for the mass difference between $\mathrm{D}_{2}$ and $\mathrm{D}_{2} \mathrm{O}$ the signal levels shown in Fig. 9 and 10 have been multiplied by 5 to permit comparison with the $\nu_{2} O$ release tests; (runs 4 and 5). Nevertheless, the $D_{2}$ release rates are much lower than in the $\left[{ }_{2} \mathrm{O}\right.$ runs. A total of $7 \mu \mathrm{g} \mathrm{D}_{2} \mathrm{O}$ (equivalent) was released, with all of it being observed at temperatures above $1000^{\circ} \mathrm{C}$.

Although the melting temperature of $\mathrm{UO}_{2}$ was not achieved in Runs 4-7, complete evaporation of the sample occurred. Because of the temperature gradient in the tungsten crucibles, the $\mathrm{LO}_{2}$ was found after the experiments as a solid block $-4 \mathrm{~cm}$ above the bottom of the crucible where the pellets had been originally placed. This movement of the specimens is attributed to vapor transport of the $\mathrm{NO}_{2}$, and insured complete release of $\mathrm{D}_{2}$ or $\mathrm{D}_{2} \mathrm{O}$ even though melting did not occur. According to the results of Runs 4, 5, and 7 shown in Table 1, we conclude 
that $2-4 \mu \mathrm{g} \mathrm{D}_{2} \mathrm{O} / \mathrm{g} \mathrm{UO} \mathrm{O}_{2}$ (including $\mathrm{D}_{2} \mathrm{O}$--equivalent of $\mathrm{D}_{2}$ ) are retained by $\mathrm{UO}_{2}$ pellets which are sintered in a $D_{2} O / D_{2}$ atmosphere. Because of the equilibrium $\mathrm{D}_{2}+\frac{1}{2} \mathrm{O}_{2}=\mathrm{D}_{2} \mathrm{O}$ which probably occurs during the release process, it is not known which component of the sintering gas is responsible for the retained deuterium. The $\mathrm{D}_{2}{ }^{\mathrm{O} / \mathrm{D}_{2}}$ ratio of the gases released at temperatures $>1000^{\circ} \mathrm{C}$ is between 1 and 3 , which is compatible with stoichiometric $\mathrm{vo}_{2}$ only at temperatures $>\sim 2200^{\circ} \mathrm{C}$. It therefore appears that complete equilibrium of the released $\mathrm{D}_{2} \mathrm{O}$ with the oxygen potential of the fuel does not occur. The different rates of release of $D_{2} O$ and $D_{2}$ suggest that the ratio of these two species is not controlled by decomposition processes occurring outside the specimens, i.e., on the crucible walls.

\section{REFERENCES}

1. C. N. Spalaris and F. H. Magerth, "Residual and Fission Gas Release from Uranium Dioxide", GEAP-4314 (1963).

2. A. S. Denovan, R. W. Ashley and T. H. Longhurst, "Internal Sources of Hydrogen in Unirradiated $\mathrm{NO}_{2}$ Fuel Elements", AECL-4063 (1971).

3. H. M. Ferrari, "Nitrogen Release from $\mathrm{NO}_{2}$ Pellets at Elevated Temperatures", Nuclear Science and Engineering, Vol. 17, (1963).

4. D. R. Olander, "Retention and Release of Hydrogen and Water Vapor by Urani um Dioxide", EPRI Final Report on Project RP 617-I (1978).

5. Standard Test Method for Water Absorption, Bulk Density, Apparent Porosity, and Apparent Specific Gravity of Fired Whiteware Products, ASTM G373-72.

6. The assistance of P.E. Hart of BNWL in performing these measurements is gratefully acknowledged. 
TABLE $I$ SUMMARY OF UO $_{2}$ PELLET OUTGASSING EXPERIMENTS

\begin{tabular}{|c|c|c|c|c|c|c|c|c|c|c|}
\hline \multirow[b]{2}{*}{$\begin{array}{l}\text { Run } \\
\text { No. }\end{array}$} & \multirow[b]{2}{*}{ Date } & \multicolumn{4}{|c|}{ Pellet Characteristics } & \multirow{2}{*}{$\begin{array}{c}\mathrm{D}_{2} \mathrm{O} \\
\text { loading } \\
\end{array}$} & \multirow[b]{2}{*}{$\underset{{ }^{\circ} \mathrm{C}}{\operatorname{Max}}$ Temp } & \multirow{2}{*}{\multicolumn{2}{|c|}{ 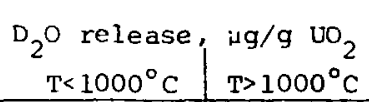 }} & \multirow[b]{2}{*}{ Comments } \\
\hline & & $\begin{array}{l}\text { Poro } \\
\text { open }\end{array}$ & $\frac{i t y, 8}{\text { closed }}$ & Sintering Conditions & $w t, g$ & & & & & \\
\hline 1 & $7 / 28$ & & & $1450^{\circ} \mathrm{C}, 2 \mathrm{hrs}$ & 16.2 & A & $i 800$ & 17.5 & 0.3 & \\
\hline 2 & $7 / 28$ & & & $1750^{\circ} \mathrm{C}, 6 \mathrm{hrs}$ & 18.2 & A & 1700 & 0 & 0 & \\
\hline 3 & $7 / 28$ & & & $1750^{\circ} \mathrm{C}, 6 \mathrm{hrs}$ & 25.2 & A & 1700 & 0 & 0 & \\
\hline 4 & $12 / 78$ & & & $1700^{\circ} \mathrm{C}, 6 \mathrm{hrs}$ & 14.2 & B & 2740 & 1.13 & 1.7 & \\
\hline 5 & $12 / 78$ & & & $1700^{\circ} \mathrm{C}, 6 \mathrm{hrs}$ & 14.2 & B & 2760 & 1.7 & 0.5 & \\
\hline 6 & $12 / 78$ & & & same as 2 & 9.1 & none & 2740 & 0.03 & 0.06 & control \\
\hline 7 & $12 / 78$ & & & $1750^{\circ} \mathrm{C}, 6 \mathrm{hrs}$ & 14.2 & B & 2740 & 0 & 0.5 & $\begin{array}{l}\mathrm{D}_{2} \\
\text { release }\end{array}$ \\
\hline
\end{tabular}

A sintered in $\mathrm{H}_{2} \mathrm{O} / \mathrm{H}_{2}$; immersed in boiling $\mathrm{D}_{2} \mathrm{O}$ prior to outgassing experiment

$B$ sintered in $D_{2} O / D_{2}\left(40^{\circ} \mathrm{C}\right.$ dew point $)$

$C$ equivalent $D_{2} \mathrm{O}$ release $=D_{2}$ released $\times 5$. 
TABLE 2. POROSITY MEASUREMENTS

\begin{tabular}{|c|c|c|c|c|}
\hline \multirow[b]{2}{*}{ Sample } & \multicolumn{2}{|c|}{ Open Porosity } & Geometric & lensity \\
\hline & $\begin{array}{c}\text { This work } \\
\text { (8) }\end{array}$ & $\begin{array}{l}\text { BNWL } \\
\text { (8) }\end{array}$ & $\begin{array}{l}\text { This work } \\
\text { (g/cc) }\end{array}$ & $\begin{array}{l}\text { BNWL } \\
(g / c c)\end{array}$ \\
\hline $\mathrm{I}$ & 0.18 & 0.08 & 10.13 & 10.16 \\
\hline II & 0.13 & 0.015 & 10.20 & 10.23 \\
\hline III & 0.16 & 0.020 & 10.13 & 10.20 \\
\hline IV & 0.22 & 0.04 & 10.21 & 10.22 \\
\hline
\end{tabular}




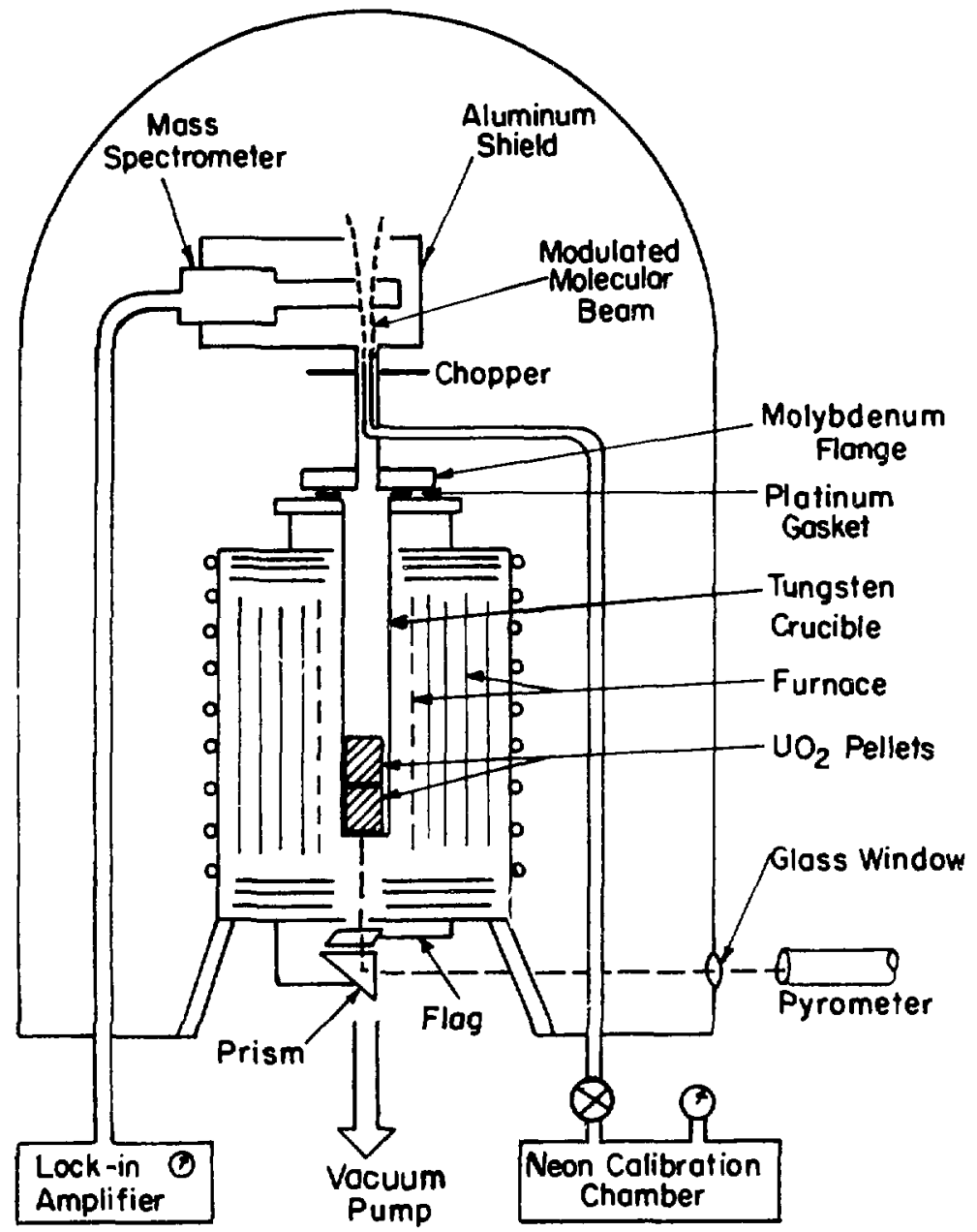

XBL 791-5625

Figure 1. Apparatus for measuring release of gases from $\mathrm{UO}_{2}$. 


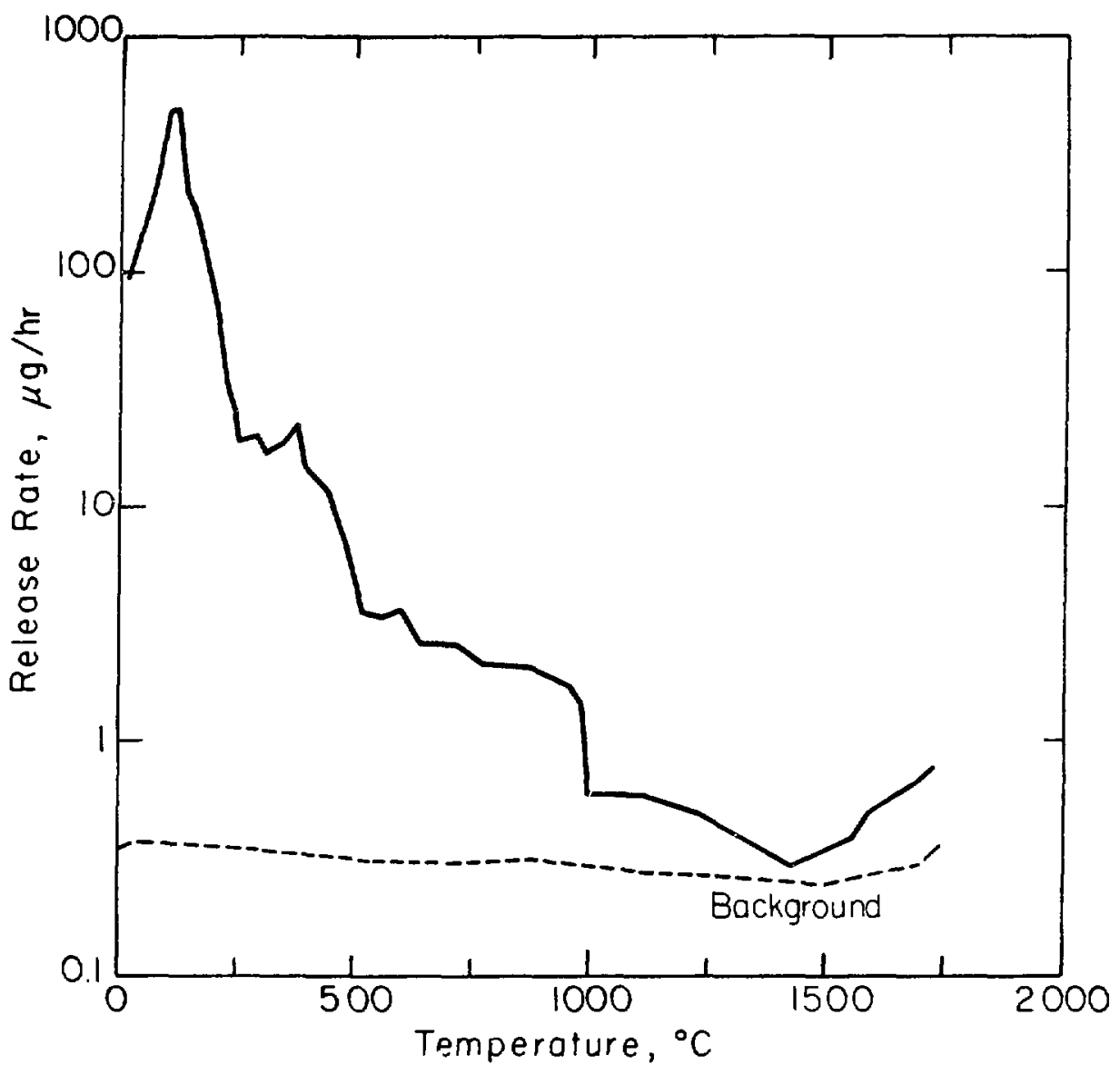

XBL 791-5552

Figure 2. Rate of release of $\mathrm{D}_{2} \mathrm{O}$ from $\mathrm{UO}_{2}$ for Run No. 1 . 


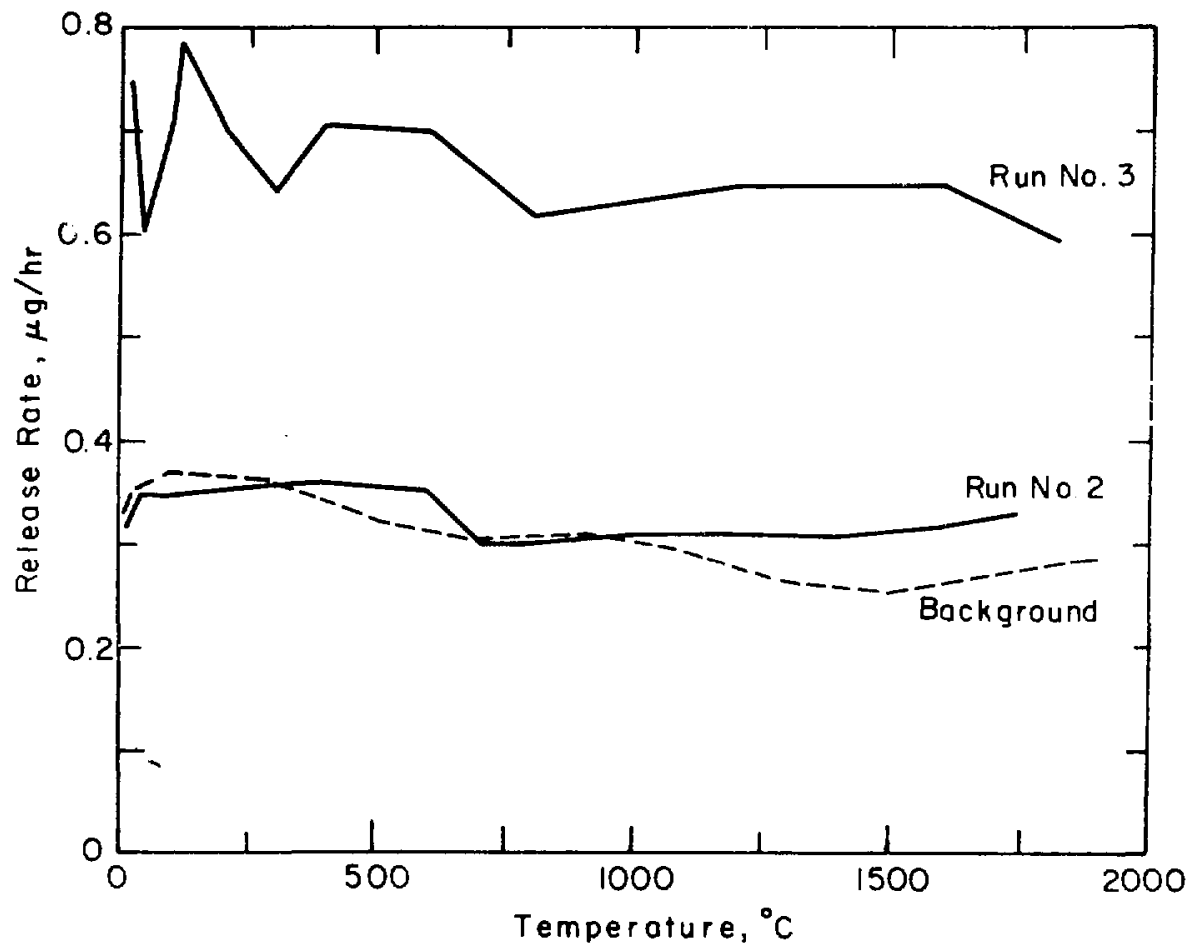

$X B L 791-5553$

Figure 3. Rate of release of $\mathrm{D}_{2} \mathrm{O}$ from $\mathrm{UO}_{2}$ for Run Nos. 2 and 3. 


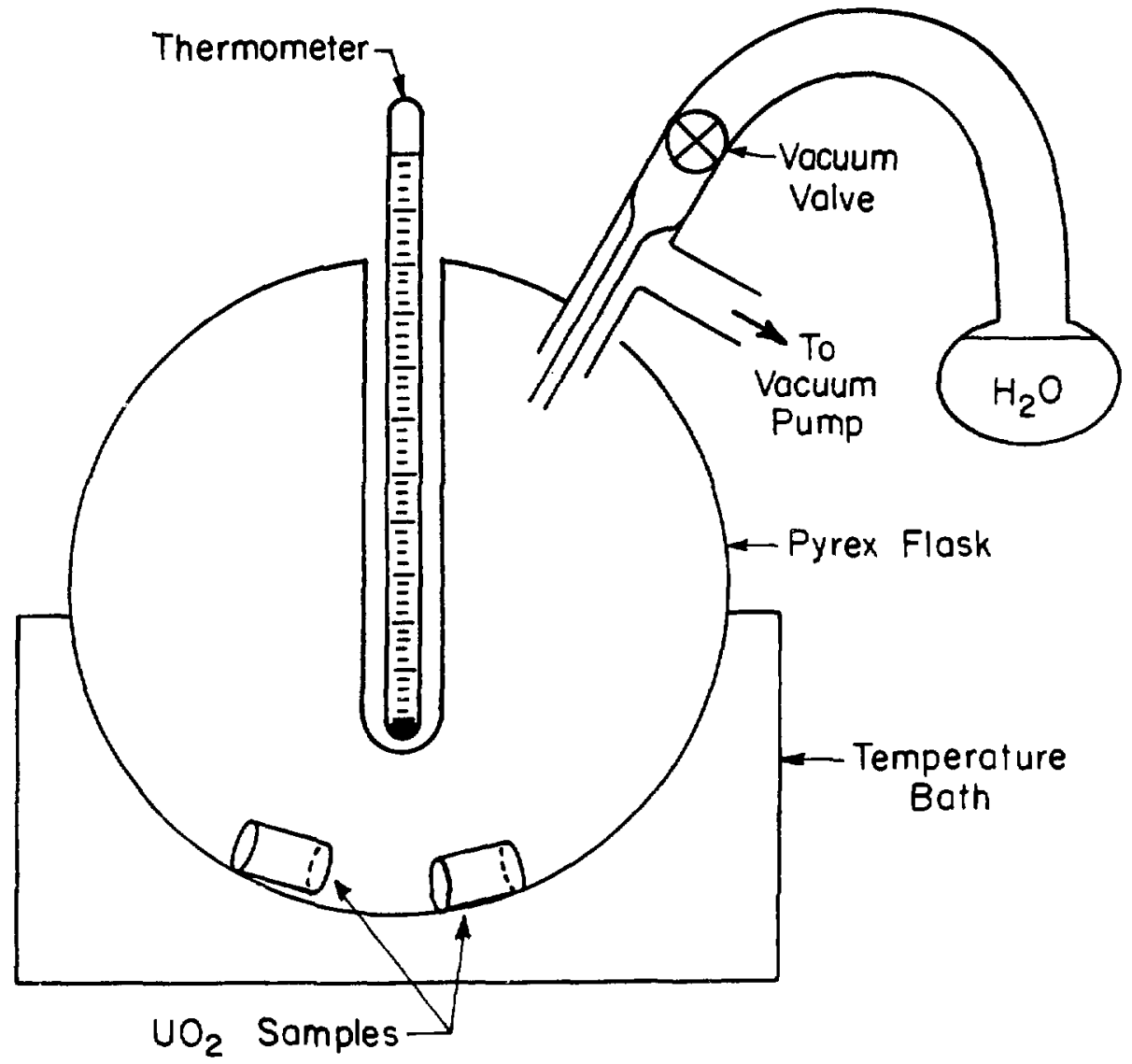

XBL $791-5624 A$

Figure 4. Apparatus for determining open porosity of $\mathrm{UO}_{2}$ samples. 


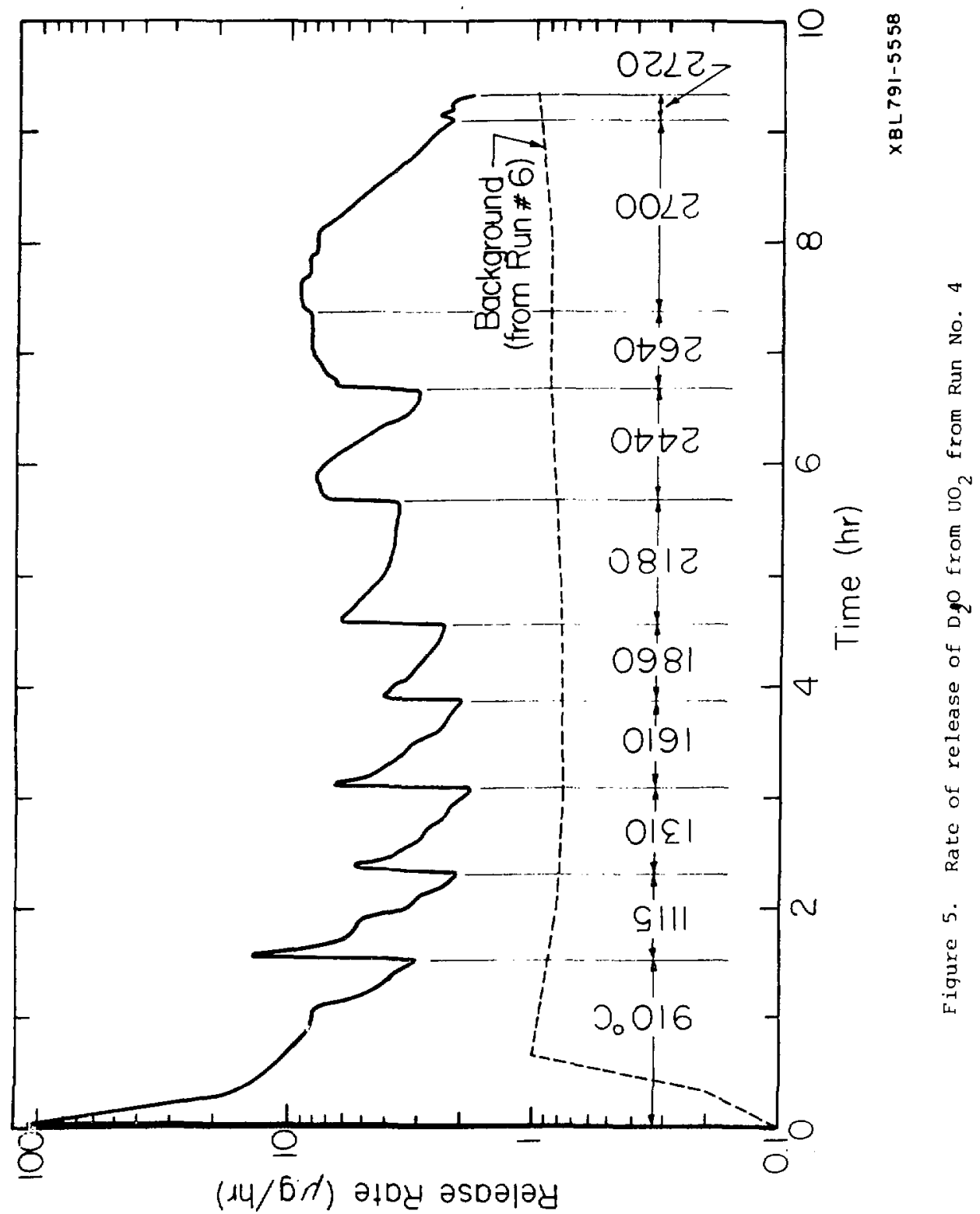




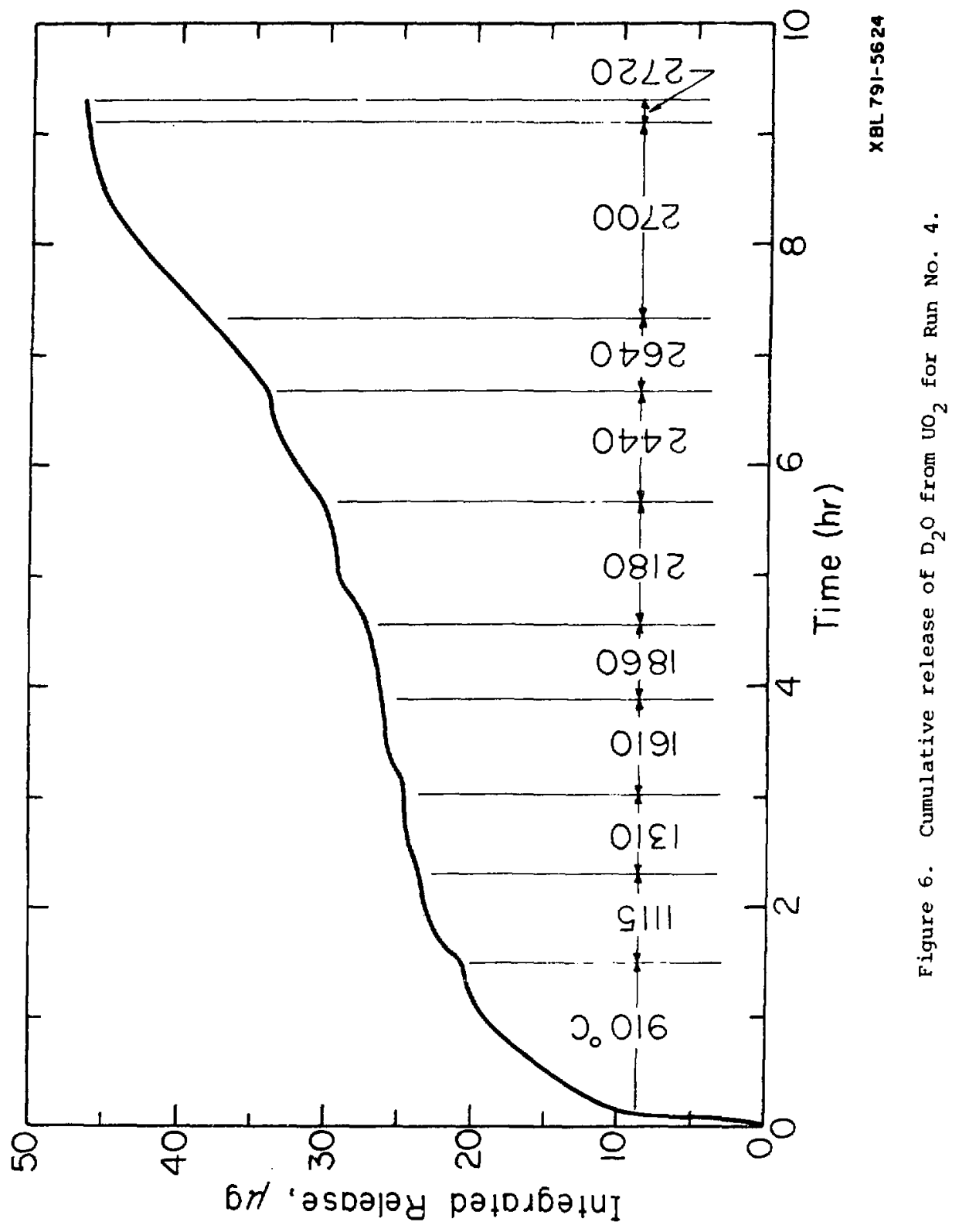




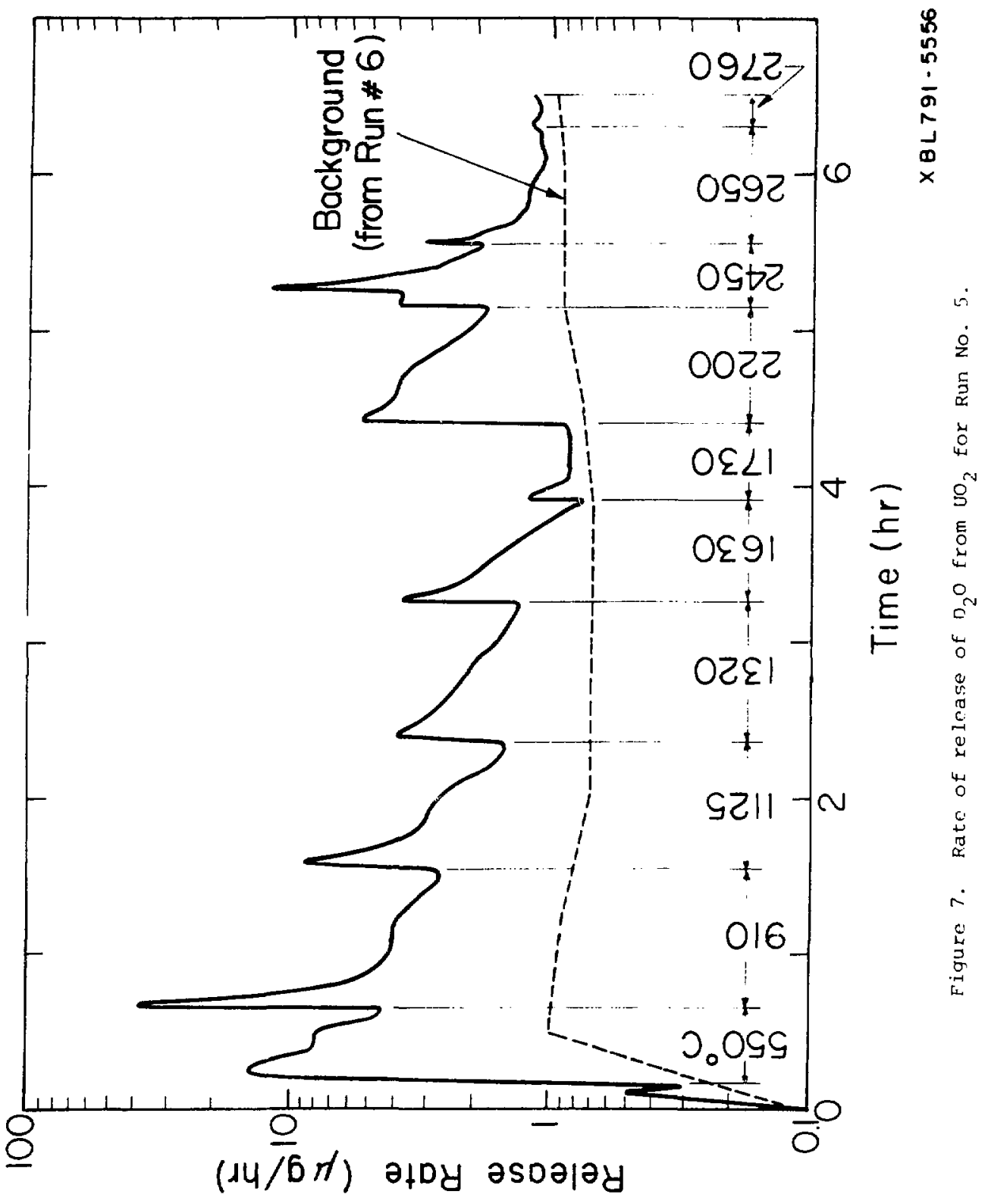




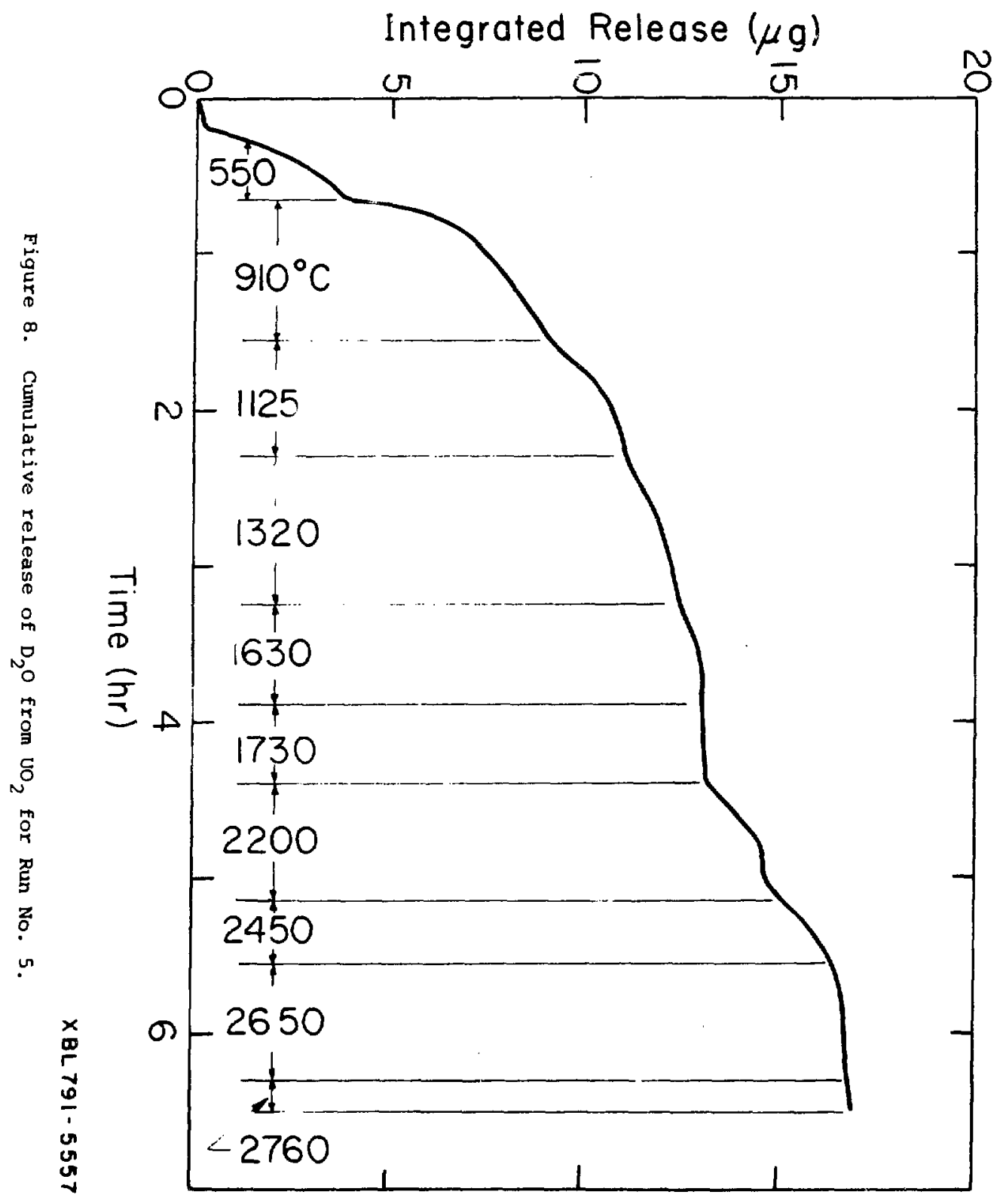




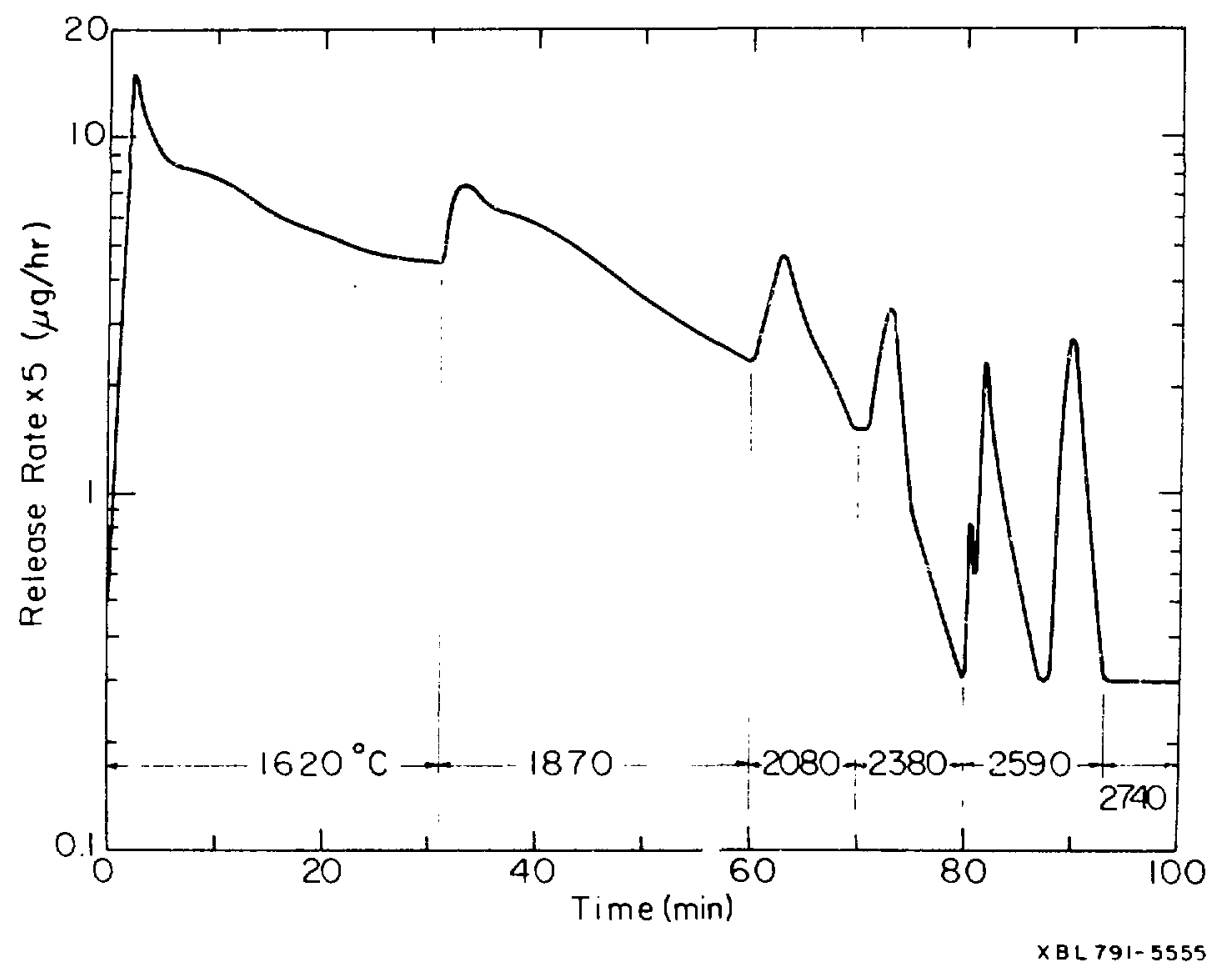

Figure 9. Rate of release of $\mathrm{D}_{2}$ from $\mathrm{UO}_{2}$ for Run No. 7. 


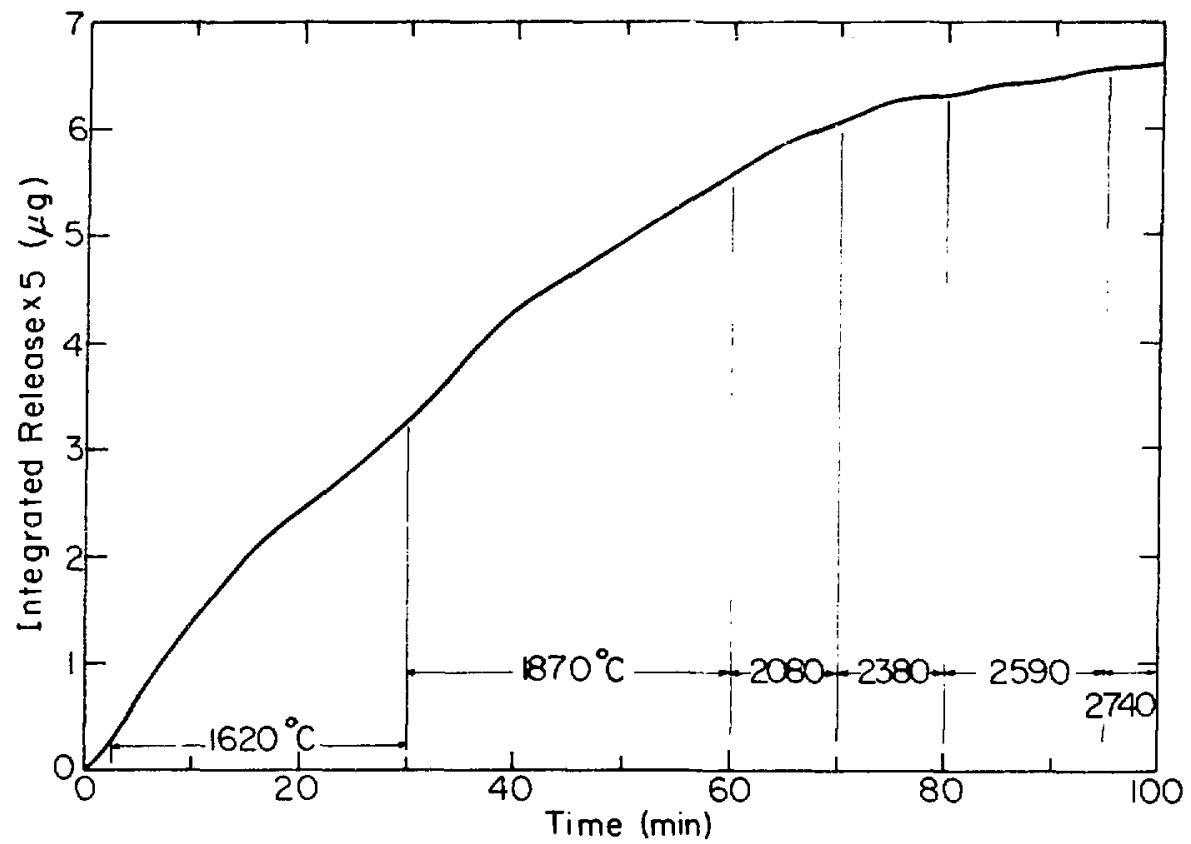

XEL 791- 5554

Figure 10. Cumulative release of $\mathrm{D}_{2}$ from $\mathrm{UO}_{2}$ for Run No. 4. 
Thermal Gradient Migration of Metallic Inclusions in $\mathrm{UO}_{2}$

by R.L. Yang

\section{Introduction}

The purpose of this experiment is to build a temperature gradient furance to study directly the migration mechanism of metailic fission product inclusions in $\mathrm{vO}_{2}$ under the influence of a temperature gradient.

\section{Experiment}

A. Sample Preparation

The $\mathrm{UO}_{2}$ used in this experiment is supplied by General Electric Vallecitos Lab. They are $95 \pm 28$ T.D. pellets, $1.3 \mathrm{~cm}$ long, with average grair, size $215 \mathrm{\mu m}$. The pellets are centerless - ground to fit into a tungsten crucible leaving a radial gal about $20 \mu \mathrm{m}$ between the specimen and the crucible. Two $0.1 \mathrm{~cm}$ thick wafers are sliced off each pellet using a thin diamond blade. Both faces of the $\mathrm{UO}_{2}$ wafers and pellets are first qround on 400, $600 \mathrm{SiC}$ paper, and finally polished with $6 \mathrm{~lm}$ diamond paste. A layers of 3-6 $\mu \mathrm{m}$ spherical tungsten powder inserted between the $\mathrm{UO}_{2}$ wafers and pellet (Fig. 1). (Which simulate the metallic fission product inclusion). The tungsten powders are deposited on the vo, surfaces by the following muthod: Two drops of Triton $\mathrm{x} 100$ (a dispersant) are added to 0.1 gram of tungsten powder in a $100 \mathrm{~m}$. beaker, then $40 \mathrm{ml}$ of methyl alcohol are added to the beaker. The beaker is then placed in an ultrasonic cleaner for 5 minutes to shake Joose any agglomerated tungsten particles. After turning of the u]trasonic cleaner. A poished $\mathrm{UO}_{2}$ pellet (or wafer) is placed in the liquid for " 20 seconds to collect a layer of tungsten powder. After taking the $\mathrm{vO}_{2}$ pellet out of the liquid, the pellet is allowed to dry slowly so that the separated tungsten powders do not coagulate during the drying 
process. A typical photomicrograph of the $\mathrm{wO}_{2}$ surface with tungsten powdrers on it is shown in Fig. 2

\section{B. Sintering the Pellet-Wafer Assemblies}

After tungsten powders are deposited, two $W_{2}$ samples, each consisting of two $\mathrm{UO}_{2}$ wafers, one $\mathrm{UO}_{2}$ pellet and two layers of tungsten powder, separated by a molybdenum disk, are pressed into a molybdenum yoko for sintering (Fig. 3). To insure that the $v \mathrm{O}_{2}$ is under high enough stress at sintering temperature to deform plastically, a 12.5 foot-pound torque is applied to the tantalum nut on the sintering yoke at room temperature before it is placed in the furance (Fig. 4). The lower part of the yoke is heated in the furnace to $ح 2000^{\circ} \mathrm{C}$ for 12 hours in $4 \% \mathrm{H}_{2}+96 \%$ argon flowing at 2.5 C.F.H. The gas is passed through magnesium perchlorate trap (to eliminate water) and heated titanium chips (to eliminate water and oxygen) before it is fed to the bell jar. The purpose of this procedure is three-fold. 1. To provide an atomsphere capable of reducing the $\mathrm{UO}_{2}$ to stochiometric or substochiometric uranium oxide, so that the urania will not oxidize the tungsten inclusions in the subsequent thermal gradient experiment. 2. To sinier the $\mathrm{UO}_{2}$ to eliminate the gap between the $\mathrm{UO}_{2}$ wafers and the pellet and to insure that the tungsten nowder is embedded in the $\mathrm{UO}_{2} \cdot 3$. To densify the $\mathrm{UO}_{2}$ sample.

Two pellet-wafer assemblies are sintered simultanously, One sample is used in the temperature gradient migration experiment and the other is sliced longitudinally, polished and checked microscopically to serve as a reference. A photomicrograph of a $\mathrm{UO}_{z}$ pellet-wafer assembly after sintering is shown in Fig. 5. 
The sintering procedure is successful in the following ways: 1 . The urania is reduced so it does not ineract with the tungsten inclusions and the tungsten crucible. 2. Thr tungsten inrlusions were well inbedded into $\mathrm{UO}_{2}$ sample after the sintering process: no voids surround the tungsten particles which might impede the motion of tungsten inclusions during subsequent thermal gradient heat treatemen:. 3. The $\mathrm{HO}_{2}$ sample undergoes 1 urrat deal of structual changes e.g., densification and grain growth, during the sintering process. The microstructure of $\mathrm{NO}_{2}$ before and after the sintering process is shown in Fig. 6 .

\section{c. Thermal Gradient Furnace}

Recause $\mathrm{UO}_{2}$ is heated in vacuum, it musit bo encapsulated to prevent 'xurstive vaporization. The pellet-wafer assemblies are contained in a tumgsten crucible with an integral top 1 id in which a $1 \mathrm{~mm}$ diameter, $6 \mathrm{~mm}$ lolug radial hole is drilled by spark discharge for temperature measurement.

A schematic: diagram of the temperature gradient furnace is shown in Fig. 7 .

After a sintered $\mathrm{UO}_{2}$ pellet-wafer assembly (7) is inserted into the tungsten crucible (5) a tantalum collar (10) is electron beam welded to the bottom of the crucible. The tungsten crejcle. with the $\mathrm{UO}_{2}$ sample in it, is then put on top of a tantalum push rod (12). The tantalum yoke(3) holds the tungsten crucible down while the stainless steel nut (16) pushes the tantalum rod against. the bottom of $\mathrm{UO}_{2}$ sample to keep it in close contact with the top of tungsten crucible. A W/W-Re thermcouple(1) is inserted into a slot in the tantalum push rod to measure the bottom temperature of $\mathrm{UO}_{2}$ sampic. To make the bottom temperature of $\mathrm{UO}_{2}$ constant, a tantalum partial sleeve ring (9) is placed between the top of tantalum push rod and the tungsten crucible. Radiation shields 
(8) and alumina insulators (14) are put between the tantalum yoke and the to tungsten crucible to keep the yoke cool.

The top of the tungsten crucible is heated by electron bombardment from a 0.020 inch tungsten filament which is $\sim 3 \mathrm{~mm}$ away from the top 1 id of the crucible. The bottom temperature is controlled by the tantalum push rod which acts as a heat sink. A stainless steel dome-shaped shield and rotary glass carousel is used to protect the viewing path from being coated, so that the top temperature can be measured by a pyrometer during the entire thermal gradient migration experiment.

\section{Experimental Procedure}

The experiment is carried out in a vacuum of $2 \times 10^{-6}$ torr. When heating the specimen, the temperature is increased slowly to avoid cracking the $\mathrm{UO}_{2}$. When the top temperature reaches $\sim 2650^{\circ} \mathrm{C}$ lat the black body hole in the crucible), the bottom temperature is ' $1200^{\circ} \mathrm{C}$. There is a $1000^{\circ} \mathrm{C} / \mathrm{cm}$ temperature gradient across the $\mathrm{UO}_{2}$ sample. The sample is held under these condition for $210 \mathrm{hrs}$. Following the thermal gradient heat treatment, the specimen is sectioned longitudinally, ground with 120 , 240, 320, 400, 600 grit SiC paper subsequently and finally polished with $6 \mathrm{\mu m}$ and $1 \mathrm{\mu m}$ diamond paste. Then, it is observed microscopically to determine themigration distance of the inclusion. The grinding/polishing process is repeated several times to obtain good statistical results by uncovering additional tungstan inclusions.

\section{Results}

Several migration experiments were carried out in the new tungsten crucible. A sketch of the $\mathrm{UO}_{2}$ specimen after thermal gradient experiment is shown in Fig. 8. A concave gap is formed between the $\mathrm{UO}_{2}$ specimen and the tungsten lid. The gap arises from the porosity in $\mathrm{UO}_{2}$ sample (which is not completely eliminated by the sintering step) and the residual cracks in between the $\mathrm{UO}_{2}$ wafers and pellet. Both of these void source migrate 
to the hot lid under the influence of thermal gradient. From the experiments, however, we saw some of the tungsten spheres migrate up to the hot region. However, migration is limited to powders on the periphery $\mathrm{l}^{\prime} 2 \mathrm{~mm}$ way from $\mathrm{UO}_{2}$ surfaces). A possible explanation for this is that due to the presence of the gap which was formed shortly after the temperature gradient is imrosed, the temperature in the central region is significantly lower than at the periphery. Therefore, powders in the central region do not migrate.

\section{Future Work}

1. The main problem is how to eliminate or reduce the gap caused by porosity migration to the top lid. There are two approaches to solve this problem. One is to melt $\mathrm{KO}_{2}$ to obtain a porosity-free $\mathrm{vO}_{2}$ sample. The other iti to sinter the $\mathrm{vO}_{2}$ sample instead of isothemally in temperature aradient in a fixture shown in Fig. 9. Undr.r the temperature "Gradient, the porosity should migrate to the hot region, thus leaving high density $\mathrm{UO}_{2}$ behind. By repeating this process several times, we hope to obtain a very low porosity $\mathrm{UO}_{2}$ sample to be used in thermal gradient migration experiment.

2. A gold foil is to be inserted between the tantalum push rod and the bottom of $\mathrm{UO}_{2}$ sample to insure the bottom temperature of $\mathrm{UO}_{2}$ is constant. 3. A stainless steel. spring (as the one shown in Fig. 9) is going to be used in thermal gradient migration experiment. When the $\mathrm{VO}_{2}$ densified leaving a gap, the stainless spring will expand and close the gap between $\mathrm{NO}_{2}$ and the lid of the tungsten crucible.

\section{v. Temperature Profile Calculation}

The two measured temperatures $\left(T_{1}\right.$ at the hole in the top lid and $T_{2}$ at thebottom of the Uo pellet) and the heat flux input by $E . R$. heating serve as a basis for calculating the temperature distribution in the specimen. This requires numerical solution of the heat 
conduction equation in the cylindrical coordinates with the non-linear boundary condition of radiation heat transfer. We calculate the temperature distribution using HEATING 5 code developed by Oak Ridge National Lab. A rirtwork of 40 lattice lines parallel to the cylindrical coordinatcs $\gamma$ and $\mathrm{z}$ were laid across the specimen. The intersection of the 1 ines form 420 nodes. The heat conduction equation is replaced by a system of finite difference heat balance equations at each node. To take into account of the difference in thermal conductivity in $\mathrm{UO}_{2}$ and tungsten,

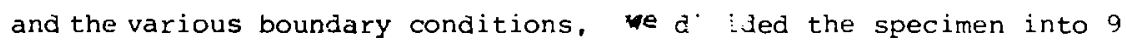
zones, with 4 different boundary conditions (Fig. 10). The thermal conductivity of tungsten is assumeo to be constant at $1 \mathrm{~W} / \mathrm{cm}^{\circ} \mathrm{K}$ in this temperature ranges. The thermal conductivity of $\mathrm{UO}_{2}$ is allowed to vary with temperature. The accuracy of this calculation depends, among other things, on the validity of the following assumptions: 1. The specimen is in good thermal contact with tungsten crucible on the sides. This should be a very good assumption because $\mathrm{UO}_{2}$ expands much more than tungsten container at this temperature and in addition any radial gap between the $\mathrm{UO}_{2}$ and tungsten crucible would be closed by evaporation after very short heating periods. 2. Thermal radiation makes up most of the heat transfer in the gap between top and $\mathrm{UO}_{2}$ sample and tungsten lid (region 4 in Fig. 10). This assumption is valid because the contribution from the conduction of the gas in the gap is small compared to radiation heat transfer at such high temperature. 3. The bottom temperature of $\mathrm{UO}_{2}$ sample is constant $\left(\mathrm{T}_{2}\right)$. 
An estimated heat flux $q$ to the top lid is used to calculate the temperature distribution, then the calculated temperature at the black body hole $\left(T_{1}\right)$ is compared with the value measured by optical pyrometer. Adjustments of the value of the iniput heat flux are made until the cisle:ulated value of $\mathrm{r}$, and the experinentally medsured value are the same. The temperature distributions in $10_{2}$ specimen, with or without a top gap, are shown in Fig. 11 and 12 respectively. 
1. filament holdex

2. high purity alumina insulator

3. stainless steel post

4. tungsten filament

5. tungsten crucible

6. black body hole ( $\ell / d \simeq 5$ )

7. sintered vo 2 pellet

8. tantalum radiation shield

9. tantalum partial sleeve

10. tantalum collar

11. W-Re thermocouple

12. tantalum push rod

13. tantalum yoke

14. alumina washer (heat insulator)

15. molybcenum collar

16. stainless steel nut

17. shield

18. rotary glass shield

19. pyrometer

20. brew furnace 


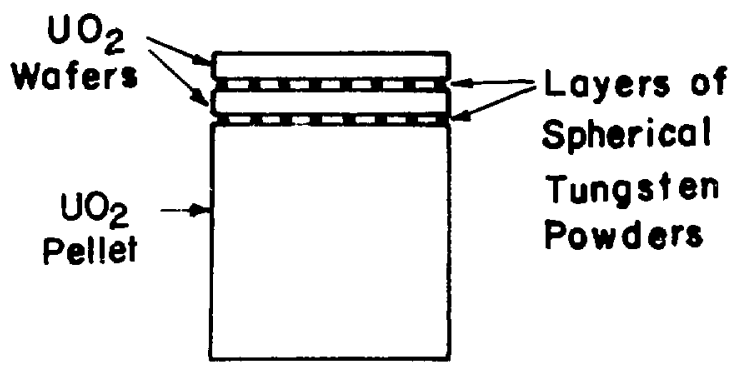

XBL $793-5860$

Fiqure 1. $\mathrm{UO}_{2}$ pellet-wafer assembly. 


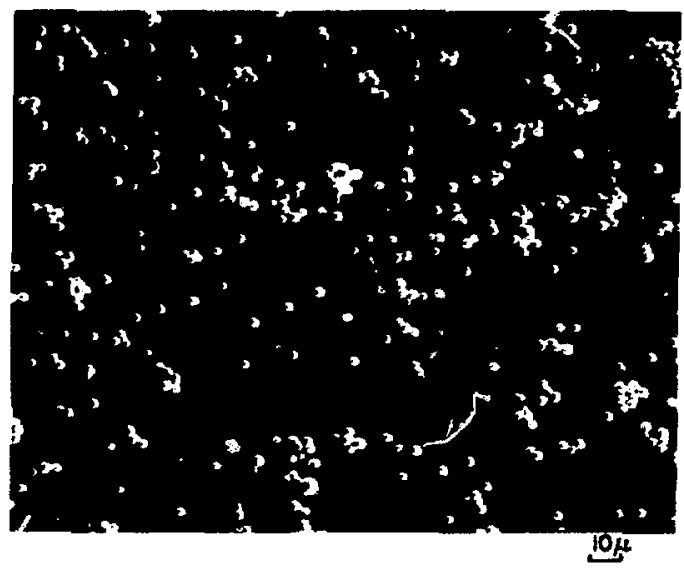

rigu'c 2. Photomicrograph of :W, surface with tuncsiten powders deposited on the surface. 


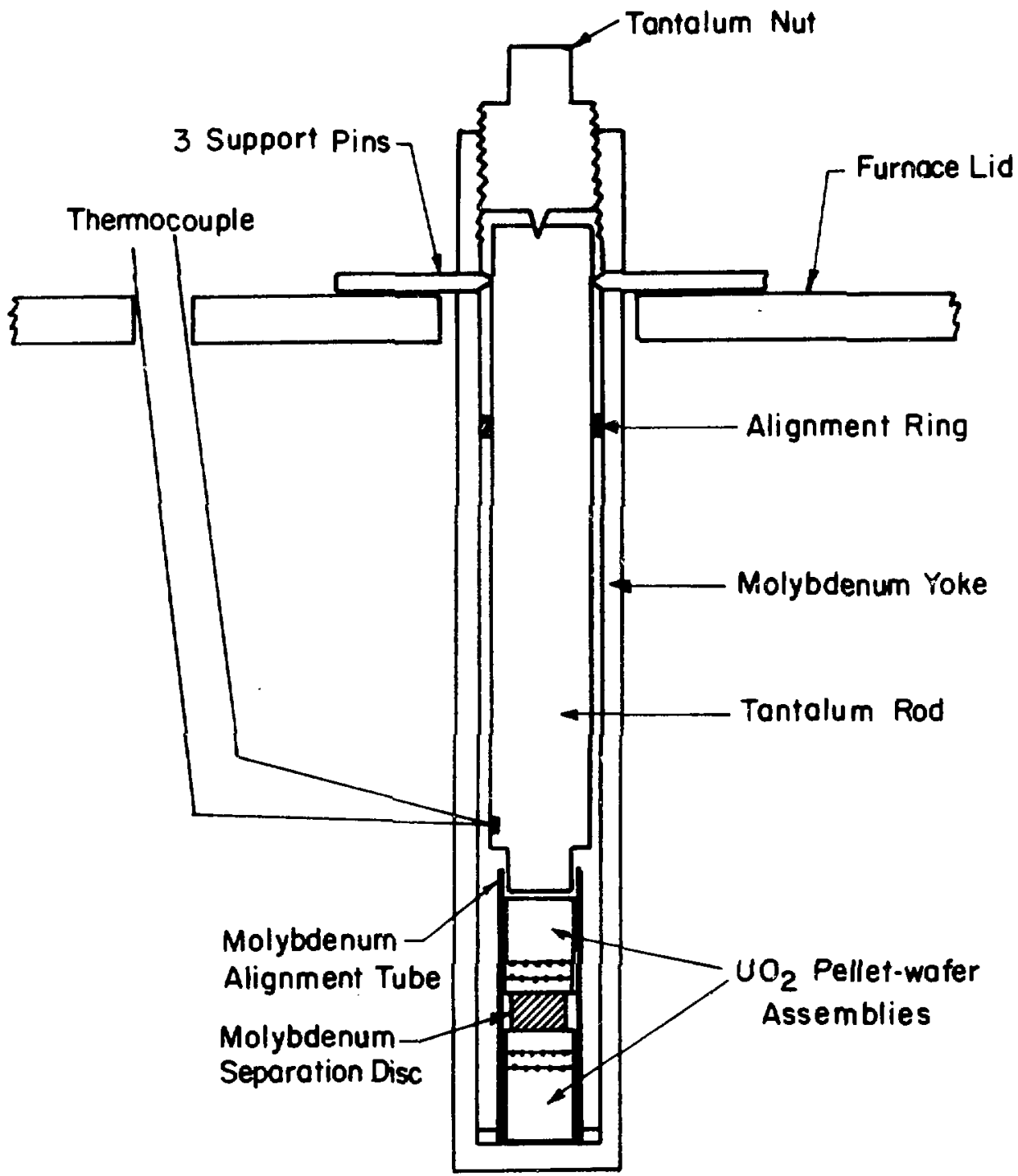

XBL 793-5861

Figure 3. Sintering yoke for $\mathrm{vo}_{2}$ pellet-wafer assembly. 


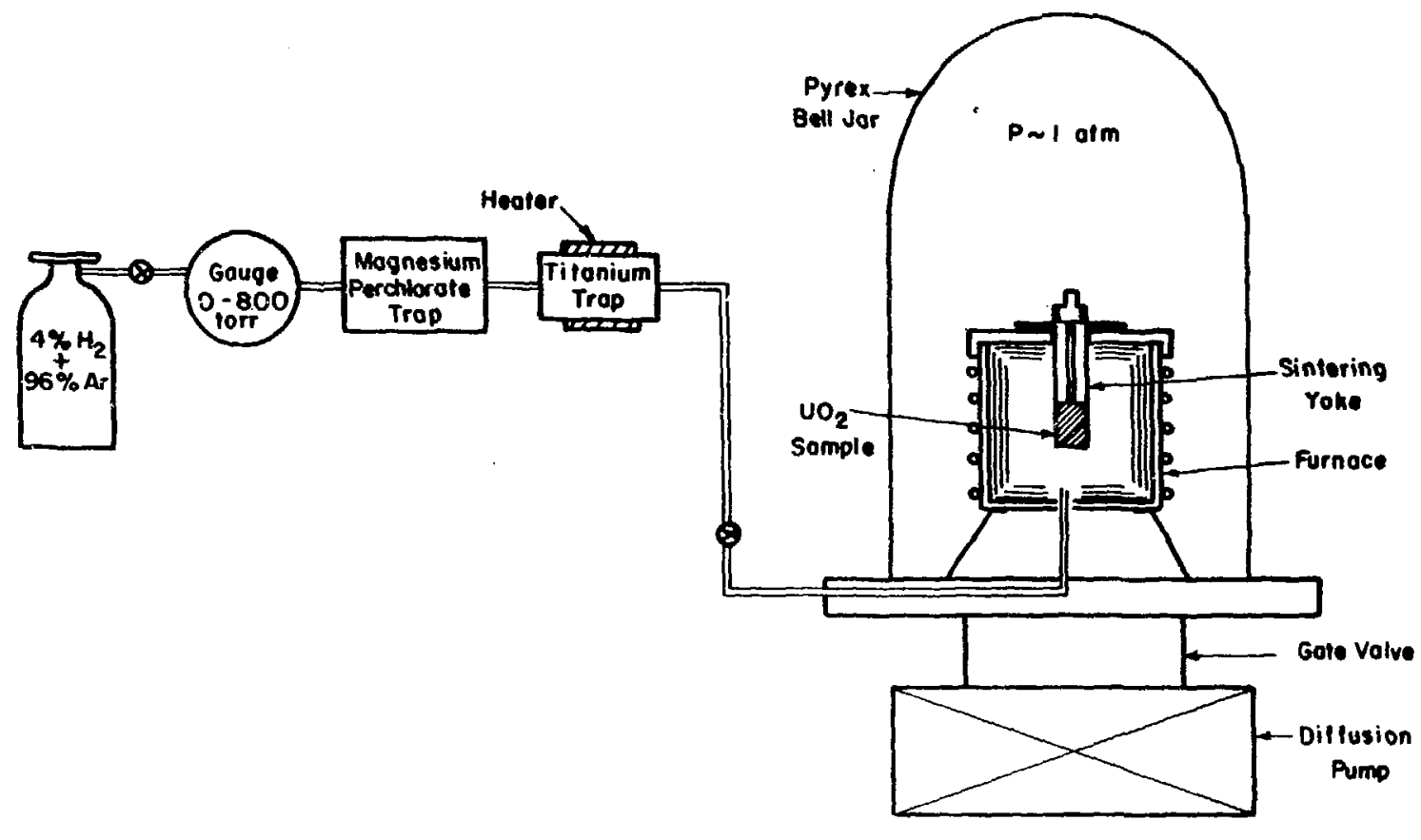

XBL 793-5862

Figure 4. Apparatus for $\mathrm{UO}_{2}$ sintering. 


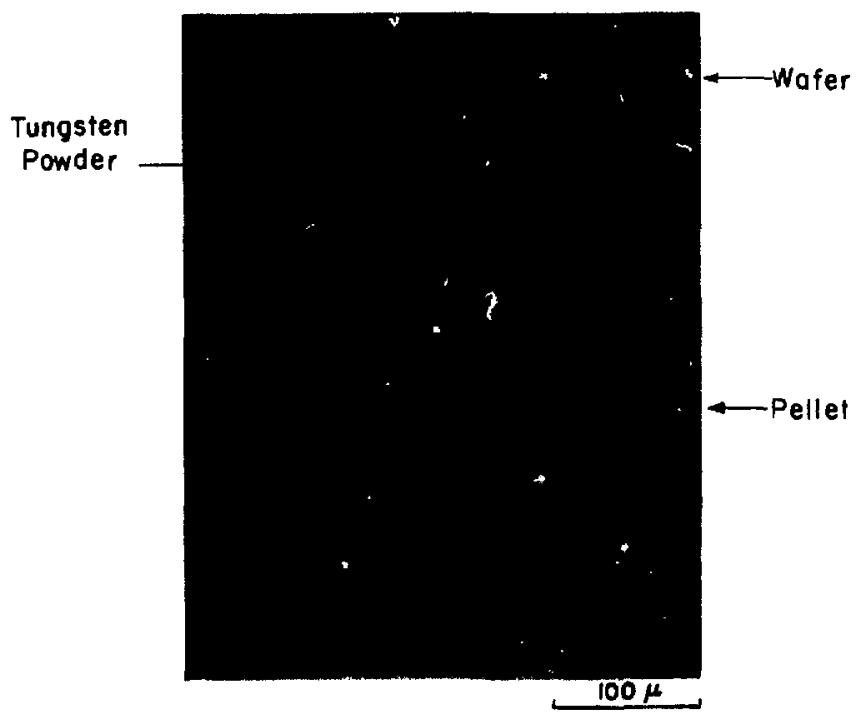

Figure s. Microrticucture longiludinal cross section of vo, palletpellet-wafer assembly after sintriting. 


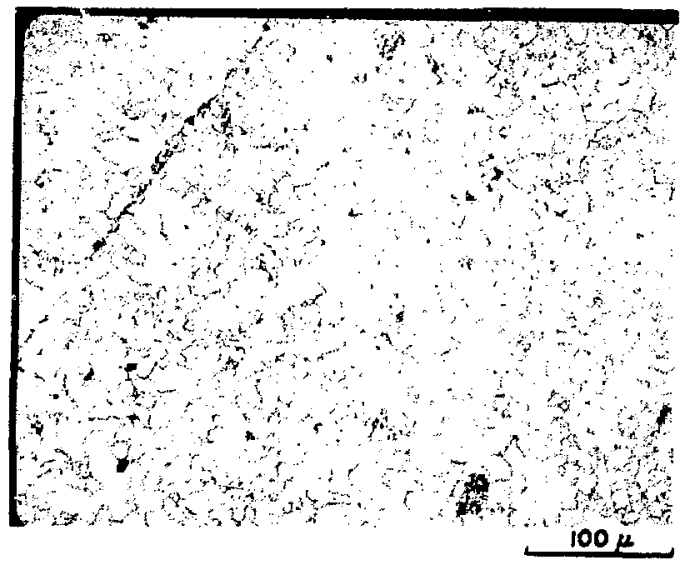

b) Microstructure of $\mathrm{UO}_{2}$ Before Sintering

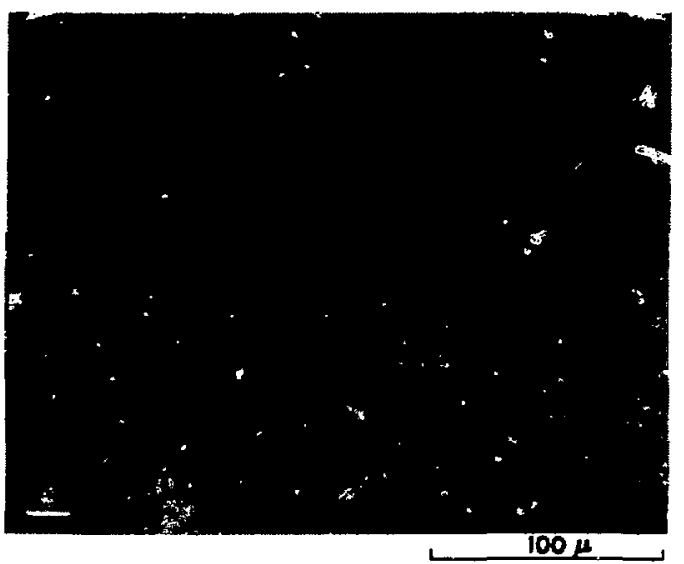

(b) Microstruct wre of $\mathrm{UO}_{2}$ After Sintering

Figure 6. Microstructure of $\mathrm{UO}_{2}$ before and after sintering process. 


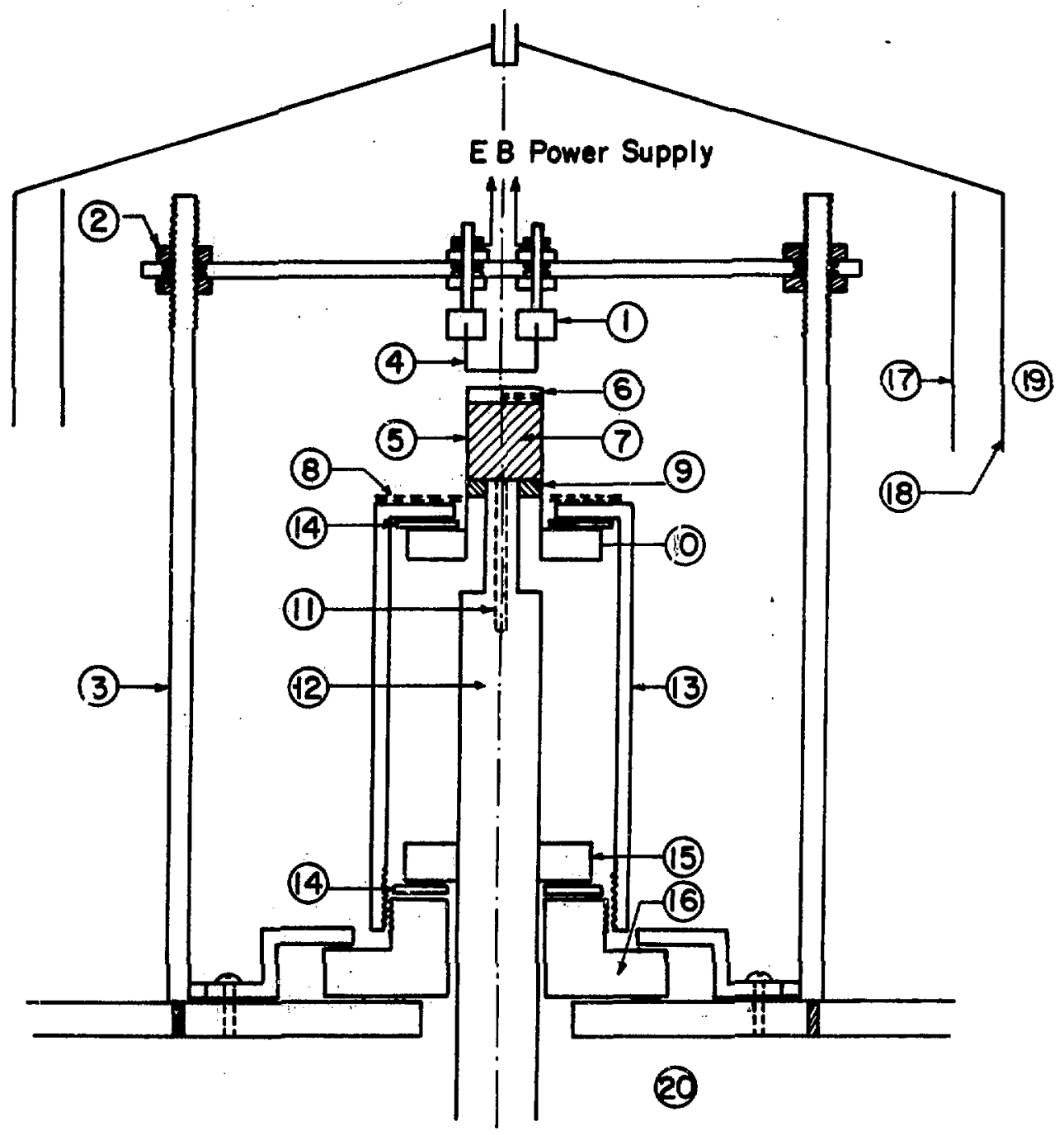

XBL 793-5863

Figure 7. Temperature gradient furnace. 


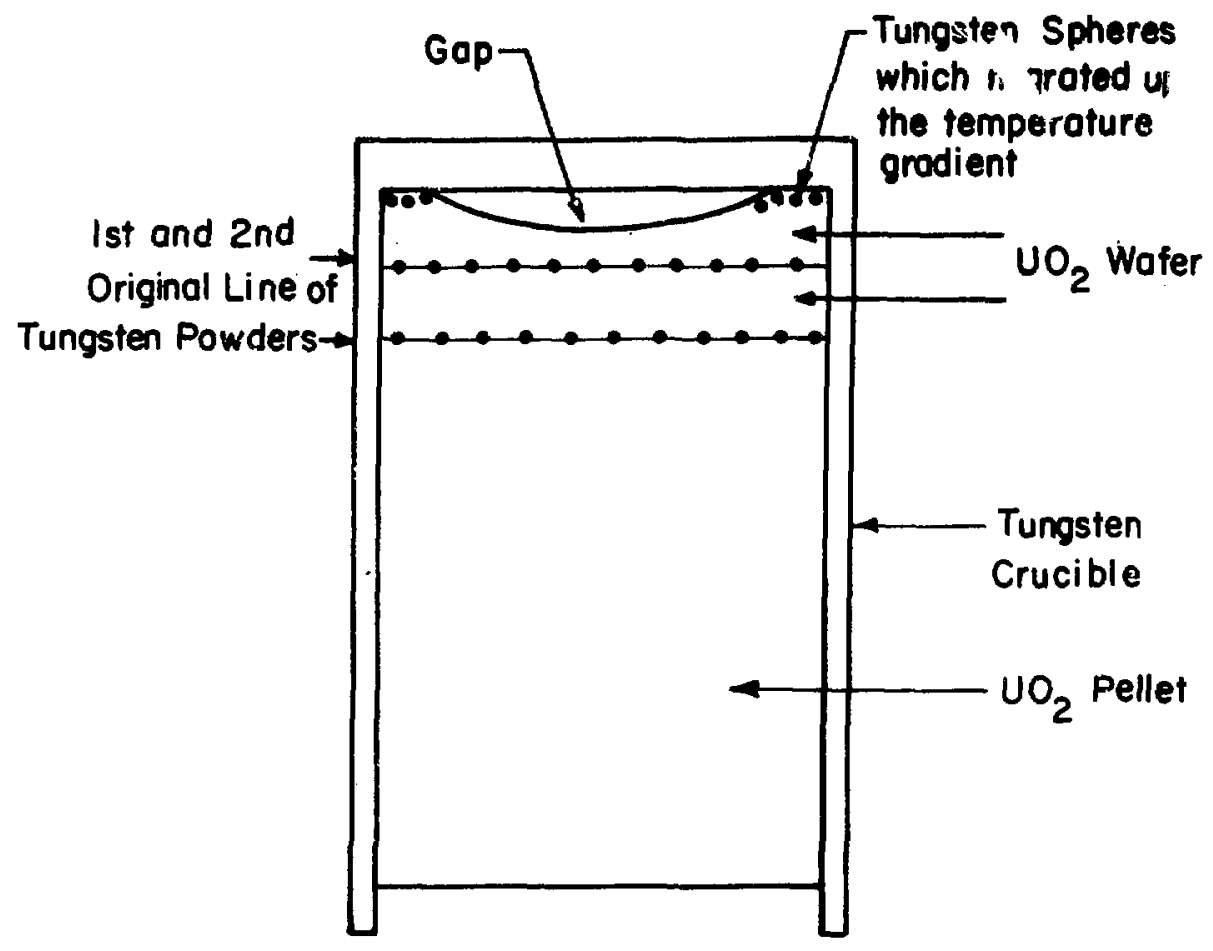

XBL793-5864

Figure 8. Schematic diagram of $\mathrm{NO}_{2}$ pellet-wafer assembly after thermal gradient migration experiment. 


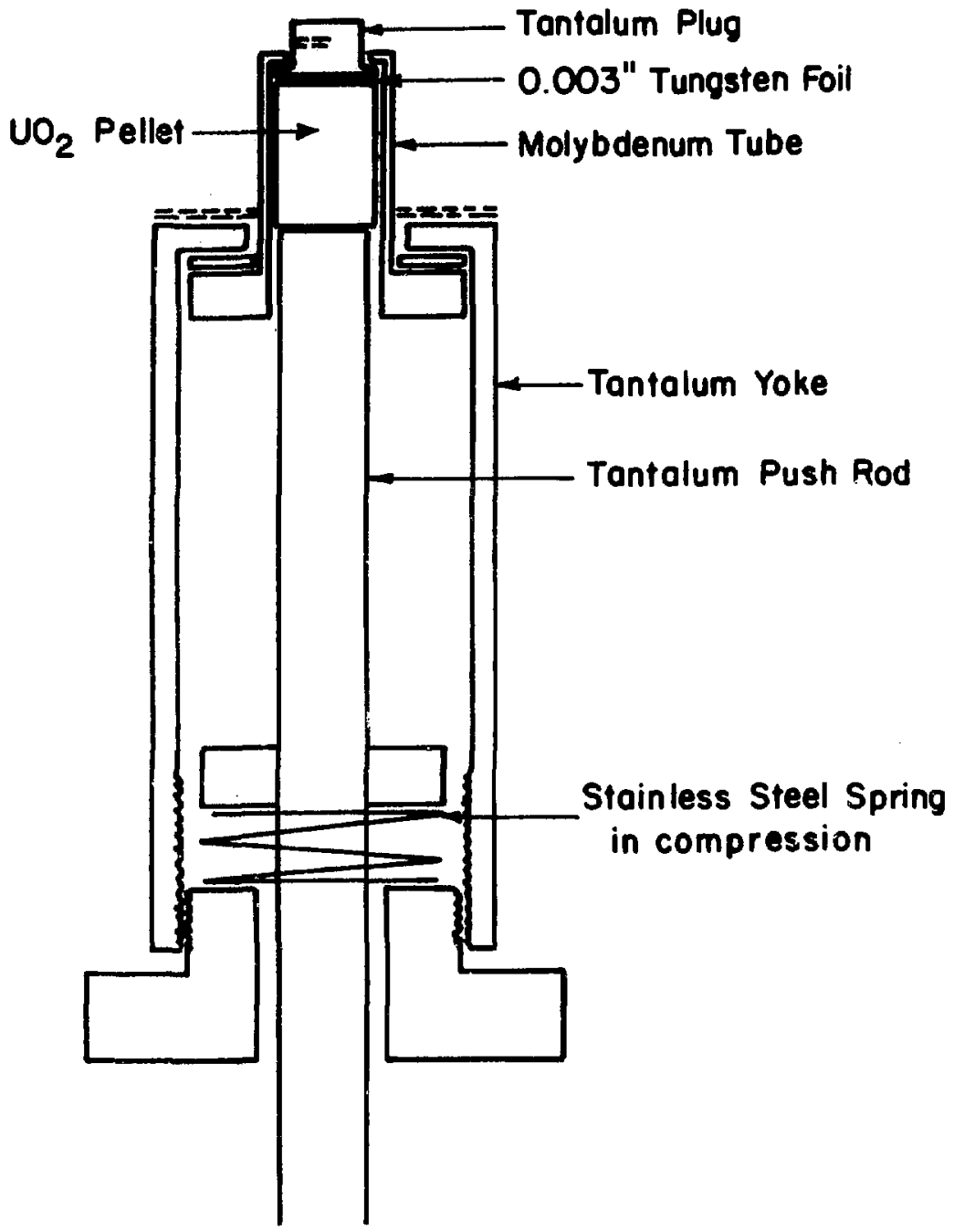

X日L 79 3-5865

Figure 9. Fixture to reduce the porosity in $\mathrm{UO}_{2}$. 


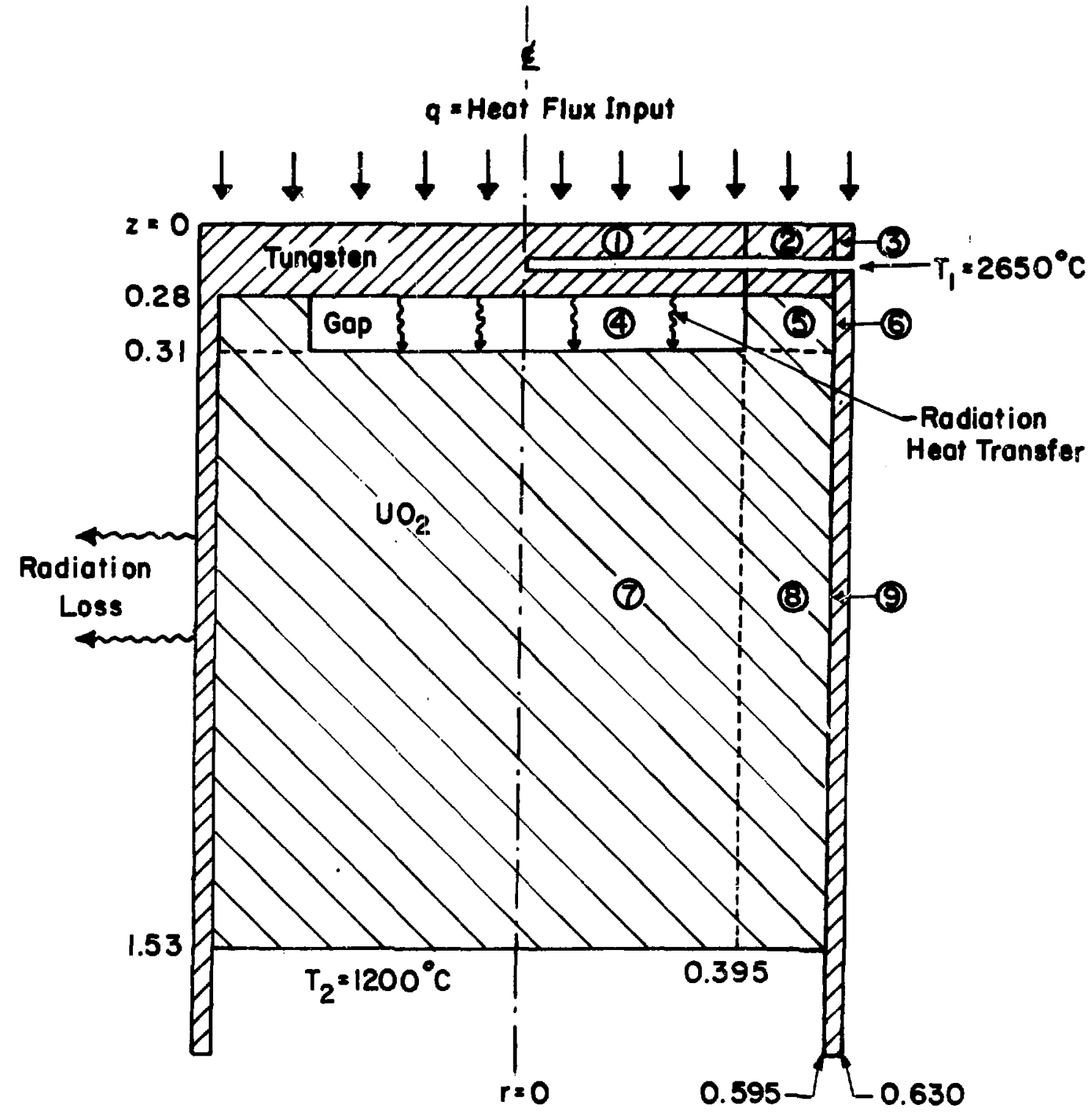

XBL793-5886

Figure 10. Cross section of the specimen for temperature calculation (all dimensions in $\mathrm{cm}$ ). 


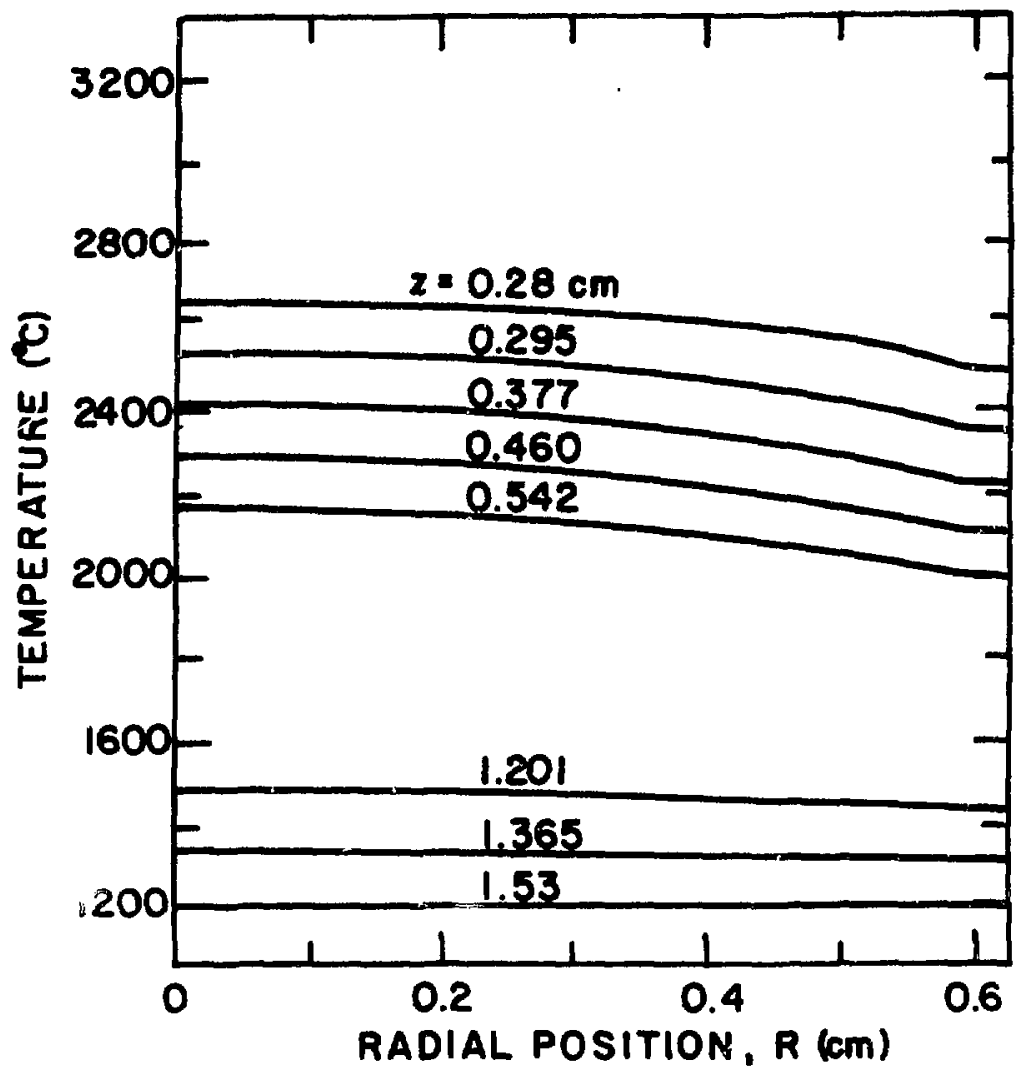

XBL 793-5866

Figure 11. Temperature distribution in $\mathrm{UO}_{2}$ when top of $\mathrm{UO}_{2}$ is in good thermal contact with tungsten lid (no gap). 


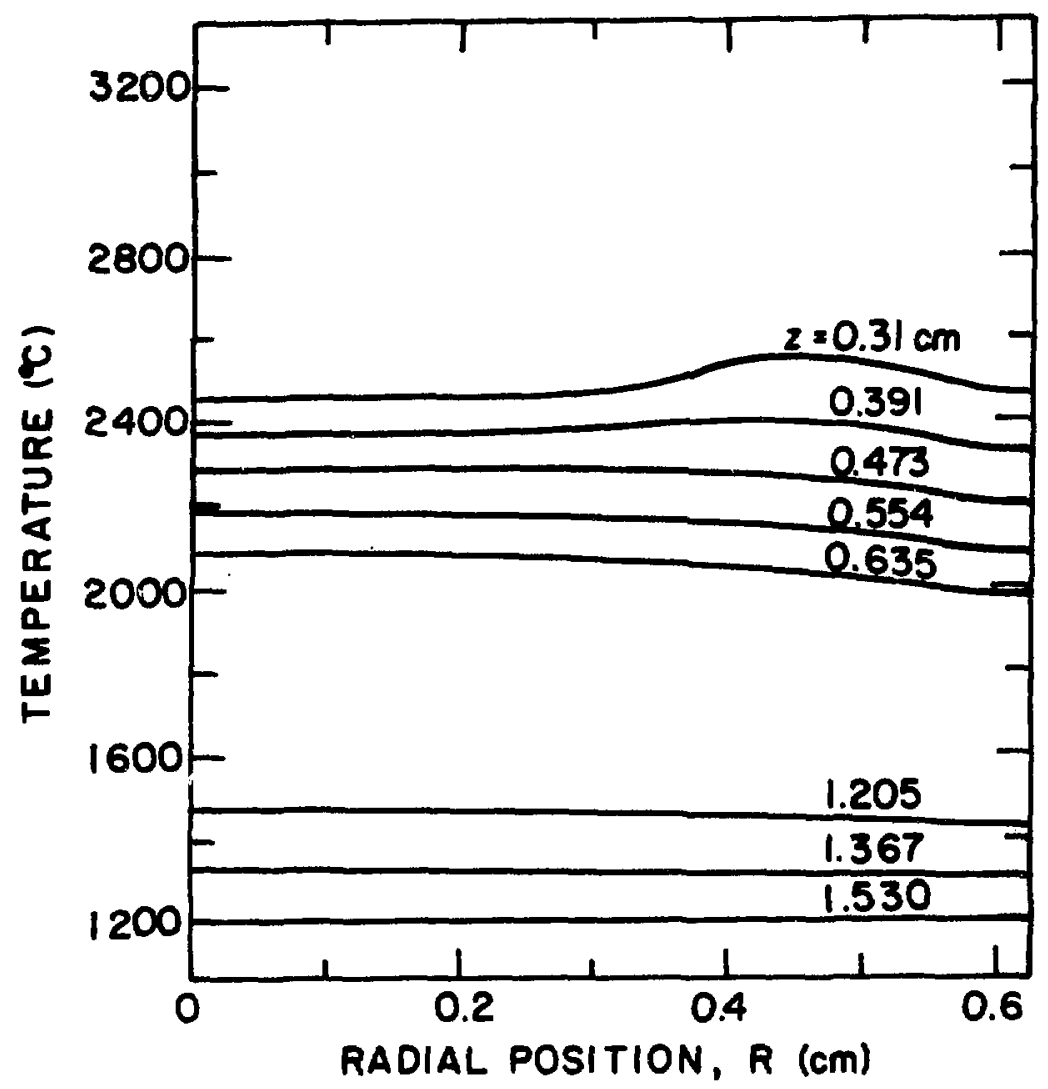

XBL 7 93-5867

Figure 12. Temperature distribution in $\mathrm{UO}_{2}$ when there is a central void formed on top of $\mathrm{UO}_{2}$ (geometry of Fig. 10). 
Molecular Beam Studies of Atomic Hydrogen Reduction of Oxides By Douglas F. Dooley

\section{Introduction}

Oxide insulator materials exposed to the hot hydrogen fuel of a CTR plasma will be subject to chemical as well as physical corrosion $(1,2)$. To better understand the nature of the chemical attack, the reduction reactions of thermal atomic hydrogen with refractory oxides, $\mathrm{NO}_{2}$ and $\mathrm{Al}_{2} \mathrm{O}_{3}$, are being studied by the modulated molecular beam method $(3,4)$.

Although previous studies have made qualitative observations of atomic hydrogen/oxide reactions and other have predicted corrosion rates based on thermodynamic equilibrium models, there is little information about the elementary reaction steps which comprise the overall reduction reaction and the values of the associated rate constants. This information can be obtained by modulated molecular beam mass spectrometry, which is a technique of studying heterogeneous chemical reactions in a detail not attainable by conventional chemical kinetic experiments.

II . Experimenta1

$\mathrm{D}_{2}$ rather than $\mathrm{H}_{2}$ is used as a reactant. The lower mass spectrometer noise background at the $\mathrm{D}_{2} \mathrm{O}$ (mass 20) peak compared to the $\mathrm{H}_{2} \mathrm{O}$ (mass 18) peak allows measurement of lower reaction probabilities in less time with high precisiou.

A palladium "diffusion filter" has been installed on the reactant deuterium gas line to eliminate beam impurities.

A beam monitor mass spectometer has been installed on the molecular beam axis. Monitoring the beam indicates that the impurity content is below the detection linit ( 1 part in $10^{5}$ ). The purpose of the direct - line mass spectrometer is to measure the $D$ to $D_{2}$ ratio in the reactant beam directly rather than by the other mass spectrometer in the system which detects scattered 
species only. The $\mathrm{D} / \mathrm{D}_{2}$ ratio in the reflected beam, in general, is not the same as in the reactant beam since recombination of $\mathrm{D}$ atoms to form $\mathrm{D}_{2}$ maly occur on the target surface.

In the past, the deuterium effusion oven was supplied with power for electrical resistance heating through a single vacuum feedthrough which eventually failed due to current in excess of rated capacity. The subsiquent loss of vacuum resulted in damage to the tungsten oven assembly. Dual feedthroughs of greater current capacity were installed to prevent recurrence of this problem.

The reactant atomic deuterium beam formed by effusion from the thermal dissociation source is modulated by a mechanical chopper prior to striking the surface of the heated oxide target. Severe reaction between the $\mathrm{Al}_{2} \mathrm{O}_{3}$ samples, and tantulum target holders occured at hich temperature. To prevent such reactions, the oxide targets are now wrapped in rhenium foil prior to clamping in the target holder.

$\mathrm{D}_{2} \mathrm{O}$ molecules as well as other species produced by the reduction reaction are defected by a mass spectrometer. The output signal is processed by a lock-in amplifier and analyzed to determine the mechanism of the reduction reaction. III. Results

Results of the $\mathrm{vO}_{2} / \mathrm{H}$ investigation indicate that reduction of $\mathrm{NO}_{2}$ by atomic hydrogen proceeds by the production of water vapor and hypostoichiometric urania:

$$
\frac{1}{\mathrm{x}} \mathrm{UO}_{2}+2 \mathrm{H} \rightarrow \frac{1}{\mathrm{x}} \mathrm{UO}_{2-\mathrm{x}}+\mathrm{H}_{2} \mathrm{O}(\mathrm{g})
$$

The reaction probability for water production as a function of $\mathrm{UO}_{2}$ temperature was measured at a fixed $\mathrm{H}$ atom beam intensity and modulation frequency. The reaction probability increased from the noise level at low temperatures to a high temperature plateau at $1300^{\circ} \mathrm{C}$. At the plateau, approximately one $\mathrm{H}$ atom out of seven striking the surface undergoes reaction and returns to the gas phase as water. 
Data on the reduction of $\mathrm{Al}_{2} \mathrm{O}_{3}$ have been taken at temperatures from $300^{\circ} \mathrm{C}$ to $2040^{\circ} \mathrm{C}$ (the melting point) at a fixed equivalent hydrogen pressure ( $5 \times 10^{-4}$ torr). Water production by reduction of $\mathrm{Al}_{2} \mathrm{O}_{3}$ remains too low to be defected (i.e., reaction probability $\lesssim 10^{-4}$ ) until the temperature is greater than $1300^{\circ} \mathrm{C}$. The low reactivity of Al ${ }_{2} \mathrm{O}_{3}$ to $\mathrm{H}$ below $1300^{\circ} \mathrm{C}$ is consistent with the other findings $(5,6)$. At higher temperatures, both $\mathrm{H}_{2} \mathrm{O}$ anci $\mathrm{Al}$ are detected by the mass spectrometcr as gaseous reaction products. Other products, such as AlO, AlH, AlOH, $\mathrm{AlOH}, \mathrm{Al}_{2} \mathrm{O}$, and $\mathrm{Al}_{2} \mathrm{OH}$ were sought but not found. The reaction probability increases with temperature, but remains two orders of magnitude below the maximum value for the $\mathrm{UO}_{2} / \mathrm{H}$ reaction. These data indicate that the reaction proceeds according to:

$$
\frac{1}{3} \mathrm{Al}_{2} \mathrm{O}_{3}+2 \mathrm{H} \rightarrow \frac{2}{3} \mathrm{Al}(\mathrm{g})+\mathrm{H}_{2} \mathrm{O}(\mathrm{g})
$$

Contray to $\mathrm{vO}_{2}$, the range of deviation from stoichiometry of $\mathrm{Al}_{2} \mathrm{O}_{3-\mathrm{x}}$ is probably so smail that even slight reduction of $\mathrm{Al}_{2} \mathrm{O}_{3}$ requires production of the metal. Because alumina cannot be rendered hypostoichiometric, its reduction by atomic hydrogen results in production of aluminum metal.

\section{Discussion}

The relative ease which $\mathrm{UO}_{2}$ is reduced by atomic hydrogen compared with $\mathrm{Al}_{2} \mathrm{O}_{3}$ is due to two factors. The first is related to the thermochemistry of reactions (1) and (2). Although thermodynamics cannot be invoked to predict chemical kinetics, one can at least expect that thermochemically favored reactions should be the most readily observed.

A rough estimate of the ease of reducing $\mathrm{Al}_{2} \mathrm{O}_{3}$ by atomic bydrogen can be obtained from the standard free energy change of reaction (2), which is : 


$$
\Delta G_{(2)}^{\circ}=-\frac{1}{2} \Delta G_{A I_{2} O_{3}}^{\circ}-2 \Delta G_{H}^{\circ}+\Delta G_{H_{2}}^{\circ}
$$

where $\Delta \mathrm{G}_{\mathrm{H}}^{\circ}$ and $\Delta \mathrm{G}_{\mathrm{H}_{2}}^{\circ}$ are the standard free energies of formation of atomic hydrogen and water, respectively, and $\Delta \mathrm{G}_{\mathrm{Al}}^{\circ} \mathrm{O}_{3}$ is the free energy of formation of alumina (per mole of $\mathrm{O}_{2}$ ). The equilibrium oxygen pressure over the $\mathrm{Al} / \mathrm{Al}_{2} \mathrm{O}_{3}$ couple is given by: $\Delta \mathrm{G}_{\mathrm{Al}_{2} \mathrm{O}_{3}}^{\circ}=\mathrm{RTlnp}_{\mathrm{O}_{2}}$ where $\mathrm{R}$ is the gas constant. The comparable quantity of $\mathrm{UO}_{2}$ is the oxygen potential $\overline{\Delta \mathrm{G}_{\mathrm{O}_{2}}}$. Hence, the standard free energy change for reaction (1) for small $x$ is:

$$
\Delta G_{(1)}^{\circ}=-\frac{1}{2} \overline{\Delta G}_{O_{2}}-2 \Delta G_{H}^{\circ}+\Delta G_{H_{2}}^{\circ}
$$

The free energy change of reaction (la) at $1000 \mathrm{~K}$ is $-310 \mathrm{~kJ} / \mathrm{mole}$ (for $\mathrm{x}=0$ in $\left.\mathrm{JO}_{2-\mathrm{x}}\right)$, whereas that of reaction $(2 \mathrm{a})$ is $-71 \mathrm{~kJ} / \mathrm{mole}$. The fact that both of these free energy changes are substantially negative suggest that, barring kinetic restrictions, reduction of the oxides should proceed in atomic hydrogen. However, urania should be easier to reduce that alumina because removal of oxygen from $\mathrm{UO}_{2}$ does not require production of the metal.

The second feature which favors efficient reduction of UO ${ }_{2}$ but not of $\mathrm{Al}_{2} \mathrm{O}_{3}$ is the oxygen diffusivity. As the reduction reaction proceeds at the surface, oxygen depletion of the surface layers will quickly stop the reaction unless oxygen can be transported to the surface from the bulk solid. The self-diffusion coefficient of oxygen in hypostoichiometric urania has not been measured, but is probably very large (7), whereas oxygen migrition in alumina is smaller, probably by many orders of magnitude (8). Consequently, oxygen transport from the interior of the solid to the reacting surface takes place easily in $\mathrm{NO}_{2}$ but in alumina, evaporation of the aluminum metal reaction product appears to be recessary to prevent a protective scale of Al from halting the surface reduction process. 


\section{Future Experiments}

1) Fresh $\mathrm{Al}_{2} \mathrm{O}_{3}$ samples have been prepared for study at the high temperatures where the reaction probability is large. Although the mass spectrometer noise background at these elevated temperatures makes measurements difficult, attempts will be made to obtain precise phase lag as well as reaction probability data since both these quantities are necessary to properly interpret the reaction mechanism.

2) Some observers have reported enhanced reduction reactions of $\mathrm{Al}_{2} \mathrm{O}_{3}$ in the presence of $\mathrm{H}_{2} \mathrm{O}$ vapor $(9,10)$. The effect of $\mathrm{H}_{2} \mathrm{O}$ vapor on the reduction reaction will be investigated by the use of a doser to provide a D.C. source of $\mathrm{H}_{2} \mathrm{O}$ vapor to the $\mathrm{Al}_{2} \mathrm{O}_{3}$ surface.

The previous $\mathrm{UO}_{2}$ samples studici were probably hyperstochiometrje due to prolonga exposure to atmospheric oxygen and moisture. Furture experiments will investigate the effect of changing oxygen-to-metal ratio upon reactivity by using both stochiometric $\mathrm{UO}_{2}$ and hypostochiometric $\mathrm{UO}_{2}$ polycrystalline wafers as targets. A single-crystal $\mathrm{UO}_{2}$ target will also be studied to determine the effect of grain boundries and crystal orientation upon reactivity. During these exneriments, the possibility of the existeance of "roljtile uranium - bearing species from the $\mathrm{UO}_{2}$ reduction reaction will be checked.

\section{References}

1. D.M. Gruen, Chemical Effects of Thermonuclear Plasma Interactions With Insulator and Metal surfaces. J. Nucl. Mater., 53, 220 (1974).

2. J.W. Tester, R.C. Feber, C.D. Herrick, Heat Transfer and Chemical Stability Calculation For Controlled Thermonuclear Reactor (CTR), USAEC Report ILA 5328-MS (July 1973).

3. R.H. Jones, W.J. Siekhaus, J.A. Scinwurz and D.R. Olander, Investigation of Gas-Solid Reaction By Modulated Molecular Beam Mass Spectrometry, J. Vac. Sci. and Techno1. 9, 1429 (1972)

4. D.R. Olander, Heterogeneous Chemical Kinetics by Modulated Molecular Beam Mass Spectrometry, J. Colloid and Interface Sci., 58, 169 (1977) 
5. S. Veprek, Private Communication (June 16, 1978).

6. I.J. Trostel, Jr. Stability of Alumina and Zirconia in Hvarogen Ceramic Bull. 44, pp. $950 \div 952$ (1965).

7. G.E. Murch and R.J. Thorn, J. Nucl. Mat: . 71, 219 (1978).

8. Y. Oishi and W.D. Kingery, J. Chem. Phys. 33, 480 (1960).

9. D.J.M. Bevan, J.P. Shelton, J.S. Anderson, Properties of Somo Simple Oxides and Spinels at High Temperatures, J. Chem. SOC. (condon) $1948, \mathrm{pp} \cdot 1729-41$.

10. F. Sandford, E. Ericsson, Effect of Composition of Kiln Atmosphere In The Firing of Refractory Oxides, J. Am. Ceram. Soc. 4l (12) 527-31 (1958). 